CERN-EP $/ 98-40$

CERN-SL/98-12

March 11, 1998

\title{
Calibration of centre-of-mass energies at LEP1 for precise measurements of $\mathrm{Z}$ properties
}

\author{
The LEP Energy Working Group
}

R. Assmann ${ }^{1)}$, M. Böge ${ }^{1, a)}$, R. Billen ${ }^{1)}$, A. Blondel $^{2)}$, E. Bravin ${ }^{1)}$, P. Bright-Thomas ${ }^{1, b)}$, T. Camporesi ${ }^{1)}$, B. Dehning ${ }^{1)}$, A. Drees ${ }^{3)}$, G. Duckeck ${ }^{4)}$, J. Gascon ${ }^{5)}$, M. Geitz ${ }^{1, c)}$, B. Goddard ${ }^{1)}$, C.M. Hawkes ${ }^{6}$, K. Henrichsen ${ }^{1)}$, M.D. Hildreth ${ }^{1)}$, A. Hofmann ${ }^{1)}$, R. Jacobsen ${ }^{1, d)}$, M. Koratzinos ${ }^{1)}$, M. Lamont ${ }^{1)}$, E. Lancon ${ }^{7)}$, A. Lucotte ${ }^{8)}$, J. Mnich ${ }^{1)}$, G. Mugnai ${ }^{1)}$, E. Peschardt ${ }^{1)}$, M. Placidi ${ }^{1)}$, P. Puzo ${ }^{1, e)}$, G. Quast ${ }^{9)}$, P. Renton ${ }^{10)}$, L. Rolandi ${ }^{1)}$, H. Wachsmuth ${ }^{1)}$, P.S. Wells ${ }^{1)}$, J. Wenninger ${ }^{1)}$, G. Wilkinson ${ }^{1,10)}$, T. Wyatt ${ }^{11)}$, J. Yamartino ${ }^{12, f)}$, K. Yip ${ }^{10, g)}$

\begin{abstract}
The determination of the centre-of-mass energies from the LEP1 data for 1993, 1994 and 1995 is presented. Accurate knowledge of these energies is crucial in the measurement of the $\mathrm{Z}$ resonance parameters. The improved understanding of the LEP energy behaviour accumulated during the 1995 energy scan is detailed, while the 1993 and 1994 measurements are revised. For 1993 these supersede the previously published values. Additional instrumentation has allowed the detection of an unexpectedly large energy rise during physics fills. This new effect is accommodated in the modelling of the beam-energy in 1995 and propagated to the 1993 and 1994 energies. New results are reported on the magnet temperature behaviour which constitutes one of the major corrections to the average LEP energy.

The 1995 energy scan took place in conditions very different from the previous years. In particular the interaction-point specific corrections to the centre-of-mass energy in 1995 are more complicated than previously: these arise from the modified radiofrequency-system configuration and from opposite-sign vertical dispersion induced by the bunch-train mode of LEP operation.

Finally an improved evaluation of the LEP centre-of-mass energy spread is presented. This significantly improves the precision on the $\mathrm{Z}$ width.
\end{abstract}

(Submitted to The European Physical Journal C)

1) European Laboratory for Particle Physics (CERN), CH-1211 Geneva 23, Switzerland

2) Laboratoire de Physique Nucléaire et des Hautes Energies, Ecole Polytechnique, IN ${ }^{2} \mathrm{P}^{3}$-CRNS, F-91128 Palaiseau Cedex, France

3) Fachbereich Physik, University of Wuppertal, Postfach 100 127, D-42097, Wuppertal, Germany

4) L.M. Universität, Muenchen, D-85748 Garching, Germany

5) Laboratoire de Physique Nucléaire, Université de Montréal, Montréal, Quebec, H3C 3J7, Canada

6) Cavendish Laboratory, Cambridge, CB3 0HE, UK

7) CEA, DAPNIA/Service de Physique des Particules, CEA-Saclay, F-91191 Gif-sur-Yvette Cedex, France

8) LAPP, Chemin de Bellevue BP909, F-74019 Annecy-Le-Vieux Cedex, France

9) Institut für Physik, Universität Mainz, D-55099 Mainz, Germany

10) Department of Physics, University of Oxford, Keble Road, Oxford OX1 3RH, UK

11) Department of Physics, Schuster Laboratory, The University Manchester, M13 9PL, UK

12) Department of Physics, University of Wisconsin, Madison, WI 53706, USA

a) Present address: PSI - Paul Scherrer Institut, Villigen, Switzerland

b) Present address: School of Physics and Astronomy, University of Birmingham, Birmingham B15 2TT, UK

c) Present address: DESY, Notkestrasse 85, D-2000, Hamburg 52, Germany

d) Present address: Lawrence Berkeley Laboratory. Berkeley, CA 94720, USA

e) Present address: Laboratoire de l'Accélérateur Linéaire, $\mathrm{IN}^{2} \mathrm{P}^{3}$-CNRS, et Université de Paris-Sud, F-91405 Orsay Cedex, France

f) Present address: Applied Materials, Inc., Santa Clara CA 95054, USA

g) Present address: Fermilab, PO Box 500, Batavia, IL 60510, USA 
The mass, $m_{\mathrm{Z}}$, and the width, $\Gamma_{\mathrm{Z}}$, of the neutral weak boson $\mathrm{Z}$ are fundamental parameters of nature, and the large electron-positron collider, LEP, at CERN near Geneva, Switzerland is the ideal place to measure them precisely. The first phase of operation of the collider, from 1989 until 1995, known as LEP1, was devoted to the study of the characteristics of the $\mathrm{Z}$ resonance. This paper concerns the LEP1 data delivered after 1992.

In 1993 and 1995 energy scans of the $\mathrm{Z}$ resonance were performed at LEP in order to determine its mass and width. In both scans luminosity was delivered at the peak of the resonance, i.e. $91.2 \mathrm{GeV}$ centre-of-mass, and two points approximately $1.8 \mathrm{GeV}$ above and below the peak. In 1994 a large amount of data was collected at an energy close to the $\mathrm{Z}$ peak. These three datasets provide the largest part of the LEP data used to determine the parameters of the $\mathrm{Z}$ resonance, and, when the results of all experiments are combined, yield a statistical precision of about $1.3 \mathrm{MeV}$ on $m_{\mathrm{Z}}$ and $2.0 \mathrm{MeV}$ on $\Gamma_{\mathrm{Z}}$.

The natural polarization of the LEP beams [1] allows a determination of the beam energy by resonant depolarization [2] (RD) with a precision of $200 \mathrm{keV}$ [3]. A model to interpolate between RD measurements to determine the centre-of-mass energy $\left(E_{\mathrm{CM}}\right)$, at a level of accuracy comparable with the statistical precision, has been developed. The error contributions from the LEP energy uncertainties on the determination of $m_{\mathrm{Z}}$ and $\Gamma_{\mathrm{Z}}$ are approximately given by

$$
\begin{gathered}
\Delta m_{\mathrm{Z}} \approx 0.5 \Delta\left(E_{\mathrm{P}+2}+E_{\mathrm{P}-2}\right) \\
\Delta \Gamma_{\mathrm{Z}} \approx \frac{\Gamma_{\mathrm{Z}}}{\left(E_{\mathrm{P}+2}-E_{\mathrm{P}-2}\right)} \Delta\left(E_{\mathrm{P}+2}-E_{\mathrm{P}-2}\right)=0.71 \Delta\left(E_{\mathrm{P}+2}-E_{\mathrm{P}-2}\right)
\end{gathered}
$$

where $E_{\mathrm{P}-2}$ and $E_{\mathrm{P}+2}$ are the luminosity-weighted centre-of-mass energies at the two off-peak points.

The details of the calibration data collected during the scan in 1993 are described in [4]. The 1995 energy scan was performed at approximately the same energies and gave similar integrated luminosities to those of the 1993 scan. However, several additional measurements were made: Nuclear Magnetic Resonance (NMR) probes were placed directly inside LEP dipole magnets, providing considerable new insight into their behaviour; more frequent calibrations by RD were performed usually at the end of fills ${ }^{1)}$ and six times also at the beginning, whereas in 1993 all RD calibrations during physics fills were made at the end. Some problems specific to the 1995 scan, namely the operation with bunch-trains and the use of a large number of superconducting cavities which were being commissioned for the LEP 2 programme, required specific measurements. This report describes the determination of the LEP centre-of-mass energies for the 1995 scan, and, applying the further understanding gained from these measurements, a revision of the 1993 and 1994 energies is given.

The model of the accelerator energy behaviour (Section 3) has to track the two basic quantities which could cause variation of the beam-energy: the magnetic dipole field component perpendicular to the beam trajectory (Sections 6,8) and the circumference of the accelerator (Section 7). The model has been significantly improved over the one described in an earlier publication [4]. In particular the thermal behaviour of the LEP dipole magnets has been studied in great detail (Section 5). Unsuspected phenomena causing drifts of the magnetic fields have been discovered and understood. The leakage currents from the Swiss-French railway power system, referred to as parasitic currents in this paper ${ }^{2}$, perturb the field of the LEP dipoles and are the cause of the systematic drift of the accelerator dipole magnetic field. The evidence for these drifts is discussed, and the inclusion of this effect in the model is described (Section 4).

As a result of the discovery of these effects, the 1993 and 1994 energies are revised. It is explained how the parametrization of 1995 can be applied to the earlier years, and what data exist from these years to support this treatment (Section 6.1).

To obtain the centre-of-mass energy for each of the four LEP experiments requires additional corrections specific to each interaction point $^{3)}$ (IP). These arise from the stat us of the LEP radiofrequency

\footnotetext{
1) The operation of LEP is subdivided into fills. A fill, identified by a sequential number, consists, in simplified terms, of injection of the beams at $20-22 \mathrm{GeV}$ and acceleration to the final energy. A successful fill is one where, after the final tuning, the accelerated beams are put into collision mode, delivering physics events to the four experiments. A typical LEP1 fill lasts 6-10 hours. Fills are eventually dumped or switched to calibration mode when the experimental luminosities, which decrease steadily during a fill owing to particle losses, become too low.

2) These leakage currents are a well documented source of electrical nuisance and electro-chemical corrosion and are known technically as vagabond currents [5].
} 
(RF) system (Section 10.1), and from the effect of opposite-sign vertical dispersion induced by the bunchtrain operation in 1995 (Section 10.3).

The systematic errors on the centre-of-mass energy are given, together with their correlation between experiments and energy points (Section 11).

Finally, the knowledge of the beam-energy spread in 1993, 1994 and 1995 is summarized (Section 12). This is an important correction to $\Gamma_{Z}$ and to the peak cross-section. A direct measurement of the incoherent synchrotron tune has allowed a reduction of the associated systematic error by a factor of four compared to the previous determination [4].

The data collected prior to 1993 have not been reanalysed, as most of the critical monitoring information was not available or was of lower quality. The LEP energy working group considers the published analysis [6] of these data to be adequate for the determination of the centre-of-mass energies (see Section 11.2) while the determination of the centre-of-mass energy spread has been revised (see Section 12.5).

\section{The 1993, 1994 and 1995 LEP runs}

\subsection{The datasets}

In 1993 and 1995 energy scans of the $\mathrm{Z}$ resonance were made at three energy points: peak ('P'), at a centre-of-mass energy of $91.2 \mathrm{GeV}$, close to the peak of the $\mathrm{Z}$ resonance, and two points approximately $1.8 \mathrm{GeV}$ either side of the peak (' $\mathrm{P}+2$ ' and ' $\mathrm{P}-2$ ') $. \mathrm{P}-2$ and $\mathrm{P}+2$ are close to the energies of optimal sensitivity for the measurement of $\Gamma_{Z}$.

The cross sections at the two off-peak points were typically measured in adjacent fills, interspersed with measurements at the peak. This reduces any systematic biases to $m_{Z}$ and $\Gamma_{Z}$ resulting from changes in LEP operating conditions or in experimental efficiencies during the course of each year of data taking.

In 1994 LEP ran only at the peak energy. The delivered integrated luminosities and other information relevant to the energy determination are given in Table 1.

\begin{tabular}{|c|c|c|c|c|c|c|}
\hline & \multicolumn{2}{|c|}{$\mathrm{P}-2$} & \multicolumn{2}{c|}{$\mathrm{P}$} & \multicolumn{2}{c|}{$\mathrm{P}+2$} \\
\hline Year & $\int \mathrm{Ldt}$ & cal. fills & $\int \mathrm{Ldt}$ & cal. fills & $\int \mathrm{Ldt}$ & cal. fills \\
\hline 1993 & $\sim 10 \mathrm{pb}^{-1}$ & $13 / 38(35 \%)$ & $\sim 20 \mathrm{pb}^{-1}$ & $1 / 57(2 \%)$ & $\sim 10 \mathrm{pb}^{-1}$ & $11 / 31(45 \%)$ \\
1994 & & & $\sim 60 \mathrm{pb}^{-1}$ & $11 / 167(8 \%)$ & & \\
1995 & $\sim 10 \mathrm{pb}^{-1}$ & $14 / 22(69 \%)$ & $\sim 20 \mathrm{pb}^{-1}$ & $1 / 14(6 \%)$ & $\sim 10 \mathrm{pb}^{-1}$ & $13 / 23(65 \%)$ \\
\hline
\end{tabular}

Table 1: Dataset statistics per year and per energy point. The two values in the calibrated fills columns express the number of fills with at least one successful calibration divided by the total number of fills, and the percentage of integrated luminosity collected in calibrated fills.

In 1996 the LEP2 programme started, with LEP operating at energies above the W pair production threshold. The data collected during 1996, with improved instrumentation, have been used to confirm the observations of the previous years.

\subsection{Resonant depolarizations}

The best determination of the beam-energy at a particular time during a fill is by means of RD of the beams. At LEP the beam can build up a non-negligible transverse polarization through the Sokolov-Ternov mechanism [7]. The degree of polarization can be measured by the angular distribution of Compton-scattered polarized laser light. By exciting the beam with a transverse oscillating magnetic field, the transverse polarization can be destroyed when the excitation frequency matches the spin precession

3) LEP has eight straight sections numbered from 1 to 8 where only the even ones house an experiment (IP2: L3, IP4: ALEPH, IP6: OPAL, IP8: DELPHI). 
frequency. Determining the resonant depolarization frequency ${ }^{4)}$ allows a precise determination of the beam-energy $E_{\mathrm{b}}$ through

$$
\begin{aligned}
E_{\mathrm{b}} & =\frac{\nu_{\mathrm{s}} \cdot m_{\mathrm{e}} c^{2}}{\left(g_{\mathrm{e}}-2\right) / 2} \\
& =\nu_{\mathrm{s}} \cdot 440.6486(1)[\mathrm{MeV}]
\end{aligned}
$$

where $m_{\mathrm{e}}$ is the electron mass, $c$ the speed of light and $\left(g_{\mathrm{e}}-2\right) / 2$ the magnetic-moment anomaly of the electron. Here $E_{\mathrm{b}}$ is the average over the ring, weighted by the vertical magnetic field, and over the beam particles. A depolarization takes place over many thousand turns of the beams, given that a frequency sweep lasts at least 12 seconds. The precision achievable is $200 \mathrm{keV}$ [3]. The three energies used to scan the $\mathrm{Z}$ resonance correspond to spin tunes $\nu_{\mathrm{s}}$ (number of electron-spin precessions per turn of LEP) of $101.5(\mathrm{P}-2), 103.5(\mathrm{P})$ and $105.5(\mathrm{P}+2)$. The choice of the off-peak energies derives from the fact that half-integer spin tunes ensure that the beam polarization is sufficient to allow RD calibration. It is fortunate that the $\mathrm{Z}$ peak corresponds to a half-integer spin tune.

RD calibration [3] has been a regular operational tool since 1993 and this measurement has become routinely possible with separated beams. Transverse polarization with colliding beams was obtained only in special conditions, far from the physics operation mode. This limitation implies that a precise determination of the LEP average beam-energy can only be made outside physics conditions.

The time necessary to perform the measurement was considerably reduced in 1994 and 1995 as more experience was gained. In 1995 calibration was attempted at the end of each off-peak physics fill (End Of Fill - EOF - calibrations). The number of successful calibrations was larger than in 1993 and the time spent in individual calibrations was significantly reduced. Since one important component of the final error stems from the uncertainty in absolute beam energy for those physics fills without an energy calibration, this results in a significantly smaller uncertainty due to these fills. The improved operational control also allowed the successful calibration of several fills before the beams were put into collision. The six special fills in 1995, with RD both at the beginning and at the end of the fill, will be referred to as BOF-fills in the following.

Typically the RD was performed on the electron beam. From time to time (once or twice per year) the positron energy was also checked.

Table 1 shows the statistics of the calibrated physics fills for the three datasets. As explained later, special Machine Development (MD) studies, where the beam-energy is measured at different times in the same fill, played an essential role and Table 2 lists the details of such fills.

\subsection{Monitoring of the dipole magnetic field}

The method used to monitor the magnetic field in the LEP dipoles has evolved over the years. The current, which is typically $2000 \mathrm{~A}$ at peak energy, providing a field of $\approx 0.05 \mathrm{~T}$, is distributed serially to the dipoles of the ring ${ }^{5)}$. It was monitored in 1993 via a DC Current Transformer (DCCT) installed close to the power-supplies in IP2. In 1995 an additional DCCT was installed on the return lead close to IP6 to check for possible leakage to ground at the level of the coil windings along the ring. The current was measured every 15 minutes. In 1995 a special device was installed which allowed the monitoring of the current every second, for short periods of time. Short time structures have been observed at the level of $0.01 \mathrm{~A}$ (that is well below the power-supply specification of stability at the $10^{-5}$ level). There is no evidence that such structures in the main power-supply currents cause detectable short-term fluctuations nor long-term rises of the magnetic field [8].

The only measurement of the dipole field in 1993 was provided by a special reference magnet connected in series with the LEP dipoles. The reference magnet is different from the LEP dipoles: it is a high precision iron-core magnet as opposed to the cheaper concrete-reinforced dipoles used in the tunnel; it is installed in a thermally controlled environment in a surface building. To monitor changes of the field this reference magnet houses a flip-coil device and an NMR probe, which are both recorded every eight minutes. In 1994 a short section of vacuum-chamber was mounted in the magnet and a new NMR probe positioned in the middle of it to measure the field in conditions closer to those experienced by the beam.

4) The resonant condition happens when the excitation RF frequency divided by the LEP orbit frequency matches the non-integer part of the spin tune $\nu_{\mathrm{S}}$. In practice the frequency domain is swept in intervals corresponding to $0.8 \mathrm{MeV}$.

5) LEP comprises 3280 concrete-reinforced dipoles, 32 weak dipoles for special sections of the accelerator and 24 injection dipoles, providing respectively $98.37 \%, 0.19 \%$ and $1.44 \%$ of the total transverse magnetic field. 


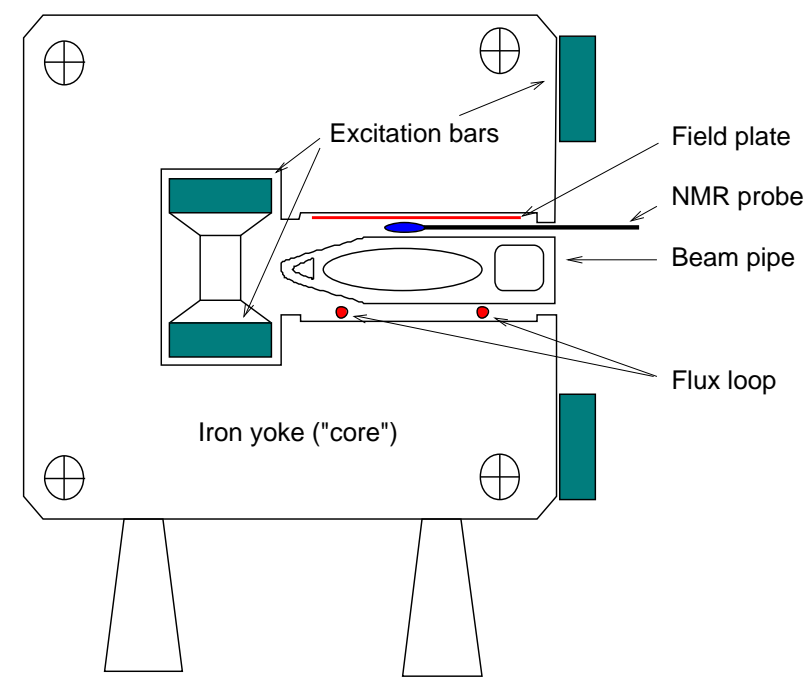

Figure 1: Diagram of a concrete-reinforced dipole cross-section. The approximate positioning of the NMR probe is also shown.

In 1995, direct measurements of the dipole field were provided by two additional NMR probes which were installed on top of the vacuum-chamber in two dipoles located in the LEP tunnel near IP4 (NMR4) and near IP8 (NMR8) as sketched in Fig. 1. In 199614 additional NMR probes were installed: in this configuration all eight of the LEP octants had a probe and in two octants strings of adjacent magnets were monitored. The measurements from 1996 are used to verify the observations made in 1995 .

\subsection{Flux-loop calibrations of the field}

A loop covering the cross-section of the dipole field [9] has been placed inside the LEP dipoles (see Fig. 1). The voltage induced in this loop while cycling the magnets provides a measurement of the field integral produced by the LEP dipoles. It is performed without beams and was repeated regularly (typically once every two weeks) to follow the overall behaviour of the accelerator field in 1993. Given the confidence in the more precise NMR measurements, and the fact that the extended magnetic cycle for the flux loop measurement was clearly affecting the magnetic history of the magnets and complicating the accelerator operation, it was decided to perform only two such measurements in 1995, at the beginning and at the end of the running period.

\subsection{Magnet cycling}

Before each physics fill the magnets are cycled at least five times (demagnetization cycle), between $+300 \mathrm{~A}$ and $+2900 \mathrm{~A}$ to reproduce the initial magnetic conditions, after which the accelerator is injected with positrons and electrons at 20-22 GeV. Ramping to the final energy was performed differently for different years. In 1993 the accelerator was ramped to $44.12 \mathrm{GeV}$ and then ramped, after a pause, to the final energy. In 1994 LEP was ramped directly to the peak energy. In 1995 the first ramp was directly to $\mathrm{P}-2$ and, when needed, a second ramp was made to $\mathrm{P}$ or $\mathrm{P}+2$. There is no evidence that this slight variation of the magnet cycles affects the energy model. In 1993 (and in 1995) a small and unexplained drift of the field of the reference magnet was measured by the NMR probe. A few erratic field jumps (believed to be caused by power-supply spikes) were also observed. Thinking that a possible cause for both these effects could be related to the fact that the dipoles work far from saturation $(0.05 \mathrm{~T}$ vs. $1 \mathrm{~T})$ and that some additional conditioning could help reduce this effect, a special magnet cycle was developed (referred to in the following as bending modulation). It consisted in modulating the main dipole current by a sequence of, typically, seven square pulses (of amplitude equivalent to roughly $10 \mathrm{MeV}$ ) over a time of two minutes, after the operational energy had been reached and before colliding the beams [8]. The bending modulation was commissioned in the first part of the 1995 run and used regularly from fill 2953 onwards ( $53 \%$ of the 1995 data was delivered after bending modulation). 


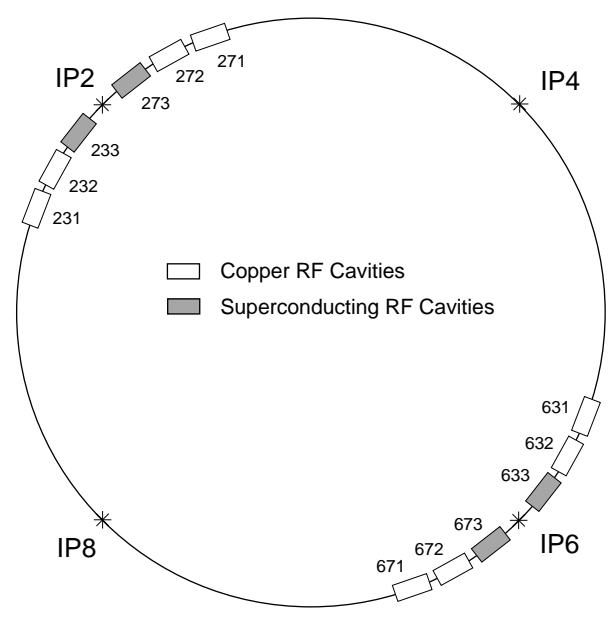

Figure 2: Schematic diagram of the RF distribution around the LEP ring during the LEP1 phase. The shaded modules show the superconducting cavities which were installed and commissioned during the 1995 energy scan. Additional superconducting cavities were installed around IP8 and IP4 only after LEP1.

\subsection{Magnet temperature}

Each accelerator octant is structured in 60 'half-cells', each comprising six dipoles, a quadrupole and a sextupole. Each octant has an independent cooling circuit. The chilled water is supplied in parallel to each half-cell where a local manifold distributes it to the dipoles, quadrupole, sextupole and vacuumchamber circuit. The temperature of the incoming and outgoing water is logged for each octant. The actual temperature of the accelerator is estimated from a set of high precision $\left(0.1^{\circ} \mathrm{C}\right)$ resistive platinum temperature probes inserted in the cores of 32 dipoles uniformly distributed (four per octant) around the ring. In 1993 and 1994 the temperature of the chilled water was kept constant in each octant. In 1995 a new control regulation was introduced aiming to maintain the core temperature as constant as possible. The reason for this was to reduce the related variations of magnetic field, leading to more stable accelerator conditions, and smaller energy corrections. The new system changes the chilled water temperature in each octant (in steps of $0.1^{\circ} \mathrm{C}$ per hour) based on the feedback of four temperature probes inserted in the dipole cores in order to maintain a preset average core temperature.

\subsection{Quadrupole-current imbalance compensation}

A different phase advance in the horizontal and vertical planes of the LEP optics means that in the quadrupole power bars running around the LEP ring at a distance of roughly $1 \mathrm{~m}$ from the vacuumchamber, there is a current difference between the circuit feeding the focusing (QF) and defocusing (QD) quadrupoles which run respectively clockwise and anticlockwise around the ring.

For the datasets considered here, LEP was operated with an optics based on a phase advance in the vertical plane (60 degrees) different from the one in the horizontal plane (90 degrees). The net current difference amounts to $33 \mathrm{~A}$, which produces a field of $6.6 \mu \mathrm{T}$ at the position of the beam in the vacuum-chamber. A compensation circuit, known as the QFQD compensation loop, has been installed around the ring in order to eliminate the effects of this stray field. This compensation circuit was used in 1993 and 1994. The control system of the power-supply for this compensation circuit proved rather unreliable, so in 1995 it was decided not to use it and instead to cope with the small additional constant component of the field.

\subsection{Radiofrequency distribution}

In 1993 and 1994 the power lost by the beam due to bremsstrahlung, about $125 \mathrm{MeV}$ per turn, was provided by the original set of copper cavities (CU-RF) distributed symmetrically along the straight sections close to IP 2 and IP6. In 1993, during the energy scan, a precondition for running off-peak was to have a stable and symmetrical RF configuration: this stability requirement minimized the spread of the 
IP-dependent RF corrections and allowed for interpolation when RF system data were missing. In 1995 the energy scan was performed concurrently with the commissioning of the first set of superconducting $\mathrm{RF}$ cavities (SC-RF) to be used in preparation for LEP2. Two SC-RF units were installed for testing in 1994, but were not used extensively, and had negligible effect on the 1994 beam-energy.

New cavities were installed around IP2 and IP6 (see Fig. 2). Most of the power was available at IP6 and the accelerator was operated for a large fraction of time with a highly asymmetric and somewhat unstable RF configuration. The recording of the RF data became essential to the calculation of the IPdependent corrections and having the recording system fully working became a precondition for running off-peak. Additional measurements were made at later dates to determine parameters for the new cavities and reduce the associated errors.

\subsection{Effects related to the LEP mode of operation}

LEP operated in 1993 and 1994 in 'pretzel' mode. Eight equally spaced bunches of electrons and positrons collided at each of the four experimental IPs. The unwanted encounters at mid-arc were avoided by causing a pretzel-like oscillation of the two beams in the horizontal plane, which provided the necessary separation where the beams crossed. The pretzel oscillation was not large enough to provide enough separation in the odd IPs so additional vertical separators were available to prevent unwanted collisions. No significant side-effect on the average centre-of-mass energy determination has been seen from this mode of operation.

In 1995 LEP was operated in 'bunch-train' mode. Four equally spaced trains of up to four bunches (separated by $247 \mathrm{~ns}$ ) crossed at each IP. All bunches in the same relative position within a train belonged to a family: the first bunch on the train belongs to family A while the fourth belongs to family D. In practice LEP operated for most of the time with only three families or less. A rearrangement of the available electrostatic separators provided a vertical separation bump around each IP to prevent unwanted collisions outside the experimental areas. The vertical separation bumps cause a non-negligible vertical dispersion at each $\mathrm{IP}^{6}$ ) which has a different sign between positrons and electrons. This effect could have been a serious problem for the determination of the centre-of-mass energy at a given IP. Having anticipated the problem, the LEP energy team was successful in maintaining it at a negligible level, mostly through a careful control of the collision offsets (Section 10.3).

\subsection{Beam position monitors and orbit correcting coils}

For a large number of quadrupoles there is a Beam Orbit Measurement (BOM)[10] monitor made of four capacitive electrodes able to measure with a precision of a few microns the relative position of the beam with respect to the centre of the quadrupole. In total there are 504 BOMs around the ring that sense the transverse position of the beam. In particular they can be used to track the average orbit deviation in any octant of LEP. The change of the average beam orbit radius $(\Delta R)$ is related to the relative change of LEP energy $(\Delta E)$ through

$$
\Delta R=D_{\mathrm{x}}^{\mathrm{BOM}} \cdot \frac{\Delta E}{E_{0}}
$$

where $D_{\mathrm{x}}^{\mathrm{BOM}}$ is the horizontal beam dispersion at the BOM and $E_{0}$ the average beam-energy. The horizontal dispersion is significant only in the curved sections of LEP (arcs) and hence the 240 BOMs in the arcs are important to monitor radial changes of LEP. The measurements from all of the BOM stations are logged whenever the accelerator operator monitors the beam trajectories (orbits), which occurs every 10 minutes or less. These sets of data are analysed to reject monitors that are not working properly for the complete data sample and that might bias the results for some orbits : typically 50 BOMs are rejected for a given year. The reproducibility of the $\Delta R$ measurement is estimated from the 1993 and 1994 datasets to be $8 \mu \mathrm{m}$ within a fill and $15 \mu \mathrm{m}$ between fills. This precision permits the use of the radial changes to monitor long-term trends (see section 7).

For most quadrupoles there is also one correcting coil (corrector) used to steer the beam vertically or horizontally through the centre of the quadrupole The correctors are weak dipole windings mounted

\footnotetext{
6) The dispersion of a beam at a given point of the orbit is the parameter describing the level of spatial ordering of particles of different momentum $D_{\mathrm{x}}=\frac{\partial x}{\partial E} \cdot E_{0}$ where $x$ is a spatial coordinate transverse to the direction of motion and $E_{0}$ is the mean (central orbit) energy. It varies along the orbit and depends on the accelerator optics configuration.
} 
next to the quadrupole. The changes of current in each corrector are logged for offline analysis (see section 8.1 .

\subsection{The ISR test facility}

In 1996 a laboratory was established in the tunnel formerly housing the Intersecting Storage Ring (ISR) accelerator in order to investigate the behaviour of the magnetic field of the LEP dipoles. Particular importance was given to studies of temperature dependence and to an accurate reproduction of the LEP configuration. In this laboratory one of the LEP concrete dipoles was assembled around a replica of the LEP beam pipe with a cooling system similar to that of LEP. The dipole excitation bars and cooling circuit could be operated to reproduce any phase of a LEP fill. A special device allowed the 'play back' of parasitic currents recorded during LEP operation.

The results of the measurements at this test facility have been essential to the understanding the detailed thermal behaviour of the LEP dipoles.

\section{$3 \quad$ The LEP energy model}

As stated earlier, precise beam-energy measurements by RD can be performed only with separated beams. A model of the accelerator behaviour is needed to correct for the differences in the beam energy between physics and RD conditions. In addition, the model is used to predict variations of beam energy with time throughout data-taking. The absolute scale of the predictions of such a model is determined by the RD measurements.

The average beam energy (as measured by $\mathrm{RD}$ ) is determined by the integral around the ring of the vertical component of the magnetic field seen by the beam. There are several contributions to this field integral: the main one comes from the field produced by the LEP dipoles which is sensitive to temperature and parasitic currents; the second is the bending field from off-centred quadrupoles which is affected by variations in the LEP circumference, in particular changes due to terrestrial tides; and finally, the additional fields generated by the quadrupole-current imbalance compensation loop and horizontalorbit correctors must be taken into account.

Determining the parameters which can influence the field integral for a ring of $27 \mathrm{~km}$ circumference has proven to be a formidable task. New understanding has been accumulated over the years about the behaviour of the dipoles, of the geology of the Geneva area and the LEP civil engineering structures. In this section the various contributions to the determination of the experimental average beam energy are discussed. The model parametrization is introduced, followed by a detailed discussion of the various model components: the thermal behaviour of the LEP dipoles, a discussion of the evidence for unforeseen field drifts and the details of how they have been parametrized, the tidal and hydrogeological effects influencing the accelerator size and the variation of the accelerator lattice.

The LEP beam-energy variation, as a function of the time $t$, is computed every 15 minutes according to the following formula:

$$
\begin{aligned}
E_{\mathrm{b}}(t)= & E_{\text {norm }}(\text { fill }) \\
& \cdot\left(1+C_{\text {rise }}\left(t_{\text {day }}, t_{\text {fill }}\right)\right) \\
& \cdot\left(1+C_{\text {T-dipole }}(t)\right) \\
& \cdot\left(1+C_{\text {tide }}(t)\right) \cdot\left(1+C_{\text {orbit }}(f i l l)\right) \\
& \cdot\left(1+C_{\text {h.corr. }}(t)\right) \cdot\left(1+C_{\mathrm{QFQD}}(t)\right)
\end{aligned}
$$

In this equation $E_{\text {norm }}$ is used for absolute normalization, whereas all other terms follow the relative energy changes. The individual effects are discussed in the following Sections and the meaning of each of the terms is explained below.

- $E_{\text {norm }}$ is different depending on whether or not the fill in question was calibrated using the $\mathrm{RD}$ method. If it was, then this normalization factor ensures that the energy of the model at the moment of the calibration equals the value of the calibration for this fill. If it was not, this term is equal to the mean normalization factor of all the calibrated fills at that energy point (Section 6.1.2). 
- $C_{\text {rise }}\left(t_{\text {day }}, t_{\text {fill }}\right)$ is the term accounting for the rise of the bending field due to the parasitic currents flowing along the beam-pipe. It is parametrized as a function of the time-of-day, $t_{\text {day }}$, and time since the magnet reached the final field, $t_{\text {fill }}$ (Section 6.1). This term is new in the analysis.

- $C_{\mathrm{T} \text {-dipole }}$ is the temperature correction for the ensemble of ring dipole magnets (Section 5 ). The understanding of this term is substantially improved in this analysis.

- $C_{\text {tide }}$ is the correction due to the effect of the Earth tides [11][12] (Section 7.1).

- $C_{\text {orbit }}$ is the correction for the deviation of the horizontal position of the orbit from a central orbit with no quadrupole bending component. This effect is calculated using an average orbit position for each fill after the expected variations for tide have been removed (Section 7.2).

- $C_{\mathrm{h} . c o r r}$ is the correction due to the setting of the horizontal correctors (Section 8.1). This term is new in the analysis.

- $C_{\mathrm{QFQD}}$ is the correction for current in the QFQD compensation loop (Section 8.2).

The actual centre-of-mass energy for a given IP is computed from

$$
E_{\mathrm{CM}}^{\mathrm{IP}}(t)=2 \cdot E_{\mathrm{b}}(t)+\Delta E_{\mathrm{RF}}(t)+\Delta E_{\mathrm{disp}}(t)+\Delta E_{\mathrm{e}^{+}}
$$

where

- $\Delta E_{\mathrm{RF}}$ is the correction, different for each IP, due to the RF system (Section 10.1).

- $\Delta E_{\text {disp }}$ is the correction (only for 1995), different for each IP, due to the combined effect of opposite-sign vertical dispersion and beam offsets (Section 10.3).

- $\Delta E_{\mathrm{e}^{+}}$accounts for the possible difference of positron and electron average energies (constant for each year).

All the corrections, with the exception of $E_{\text {norm }}$ and $C_{\text {orbit }}$, are applied according to the conditions at that particular time, whereas $E_{\text {norm }}$ and $C_{\text {orbit }}$ are applied on a fill-by-fill basis. The information is given to each experiment in the form of a file containing the modelled centre-of-mass energy at the given IP for every 15 minutes. These files are used in convolution with the experimental data to arrive at the luminosity-weighted mean energies and rms scatters which are used to determine the $\mathrm{Z}$ resonance parameters.

\section{$4 \quad$ Field rise in a fill and related observations}

In 1995, both the six BOF-fills and the tunnel NMRs indicated that, rather than remaining constant, the field of the LEP dipoles rose throughout a fill. This rise was significantly larger than that previously observed in the reference magnet.

The BOF-fills exhibited a rise in the beam-energy. The mean rise between first and last depolarization was $3.9 \mathrm{MeV}$. This mean has a significant rms scatter of $2.6 \mathrm{MeV}$ as can be seen from table 2 . Some of this scatter can be attributed to the variation in the length of the fills (between 6 and 20 hours), but the rate of energy change was not identical in all fills. Part, but not all, of this rise can be attributed to temperature effects, as detailed in Section 5, which can lead to significant drifts of the dipole field.

Throughout 1995, the NMRs also recorded an increase in the dipole field over the fills. Assuming that this can be related to the beam-energy, the field change is equivalent to a rise of a few MeV over a typical fill. The field change observed was not necessarily the same in NMR4 and NMR8; the relative rises in both varied from fill to fill, as did the absolute size of the increase.

In addition to revealing a field rise, the NMRs displayed significant short term fluctuations with typical frequencies of around $1 \mathrm{~Hz}$. This noise was found to be strongly anticorrelated between NMR4 and NMR8; this is shown in Fig.3a. Occasionally, sudden field jumps could be observed with magnitude equivalent to an energy change of up to a few $\mathrm{MeV}$.

The fluctuations in the NMRs were found to have an unexpected dependence on time of day. Much quieter behaviour was observed between midnight and about 05:00, at which point the noise resumed.

Averaged over many fills however, the behaviour was distinctive, as can be seen from the solid circles in Fig. 3d, which shows the relative rise seen by the NMR probes since the beginning of the fill. The rise is at first steep, but after about 10 hours tends to saturate. The rate of field increase per hour 


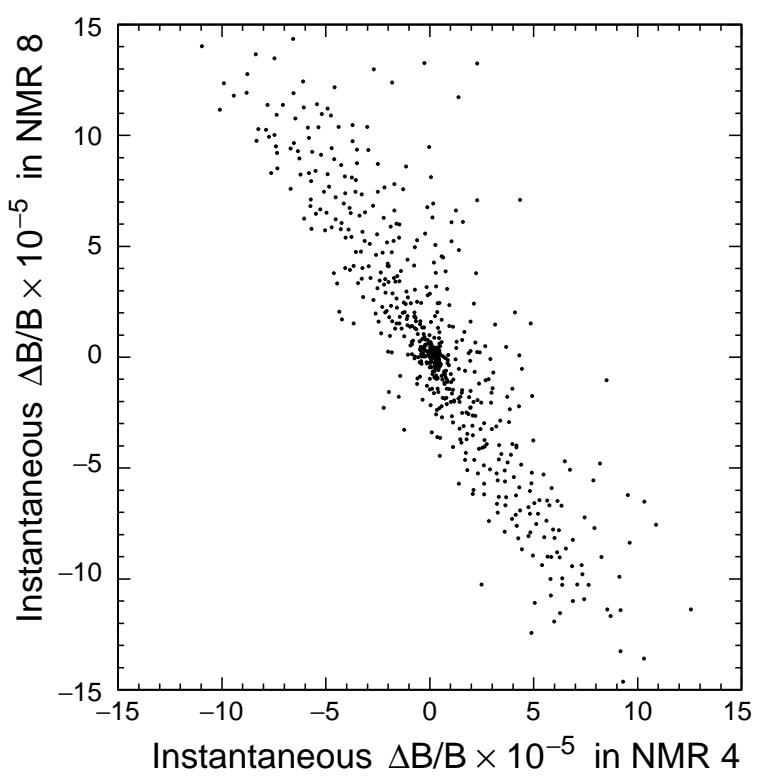

(a) Noise in NMR4 vs NMR8.

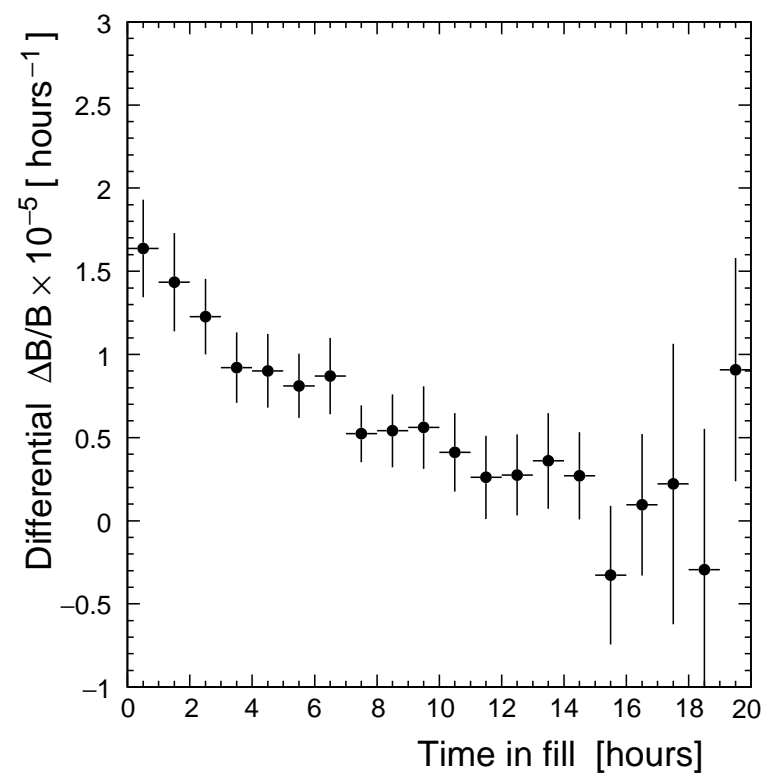

(c) Differential field rise vs. time in fill.

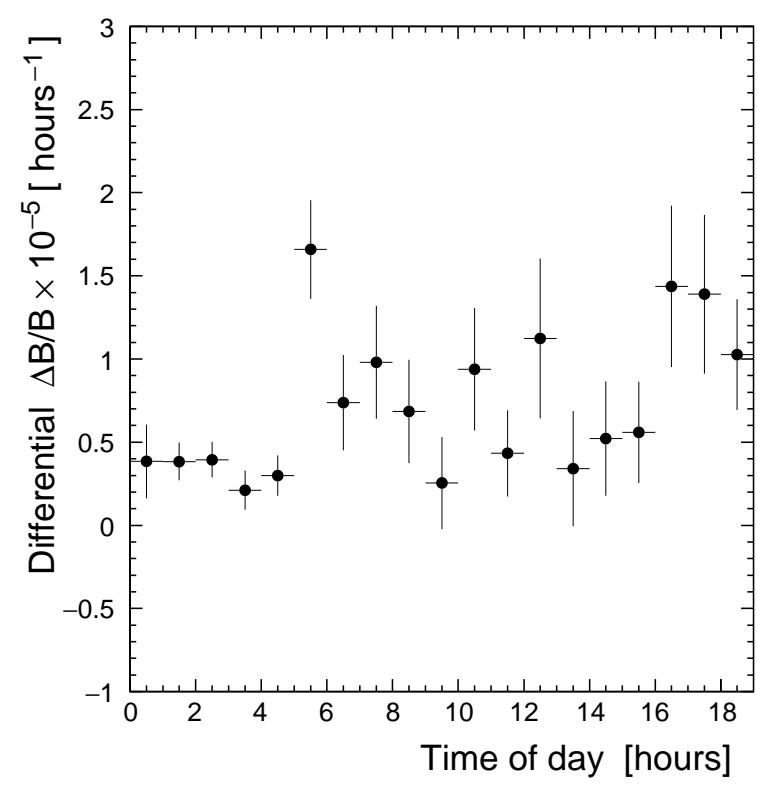

(b) Differential field rise vs. time in day.

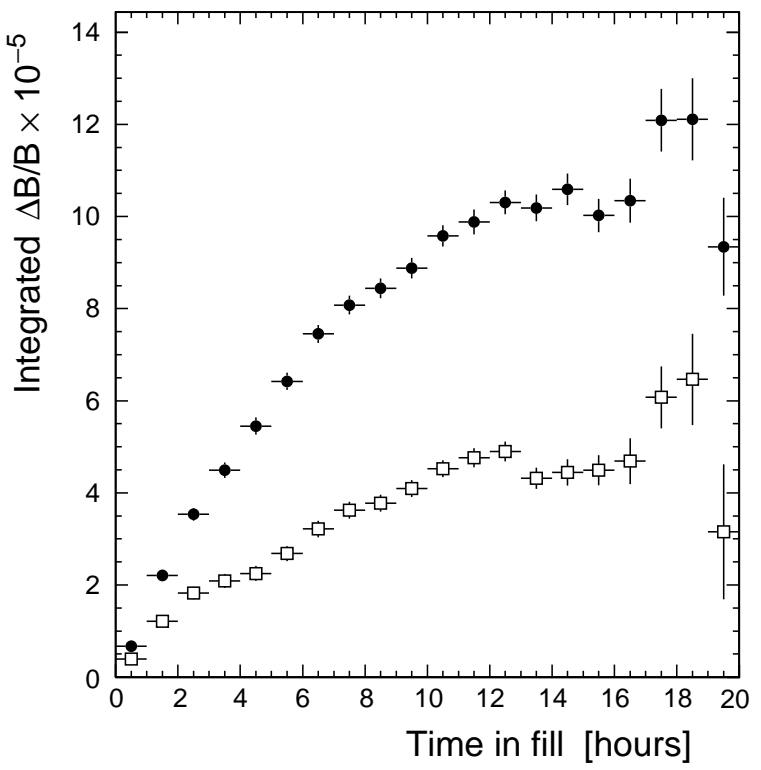

(d) Integrated field rise in fill: The black dots show the total rise, the open squares are after the subtraction of temperature effects.

Figure 3: NMR behaviour in 1995: b, c, d are obtained using NMR48 as defined in Eq. (7). 
Table 2: Fills with multiple RD calibrations, i.e. fills where the first RD and last RD were separated by at least two hours. $t_{\mathrm{f}}$ is the time of the first calibration after the start of the fill, $\Delta t$ denotes the time between the first and the last calibration, $\Delta E_{\mathrm{b}}^{\text {raw }}$ denotes the measured beam-energy difference between the last and the first calibration, $\Delta E_{\mathrm{b}}^{\text {corr }}$ is the beam-energy difference corrected for the effects of the tide, horizontal correctors, QFQD loop compensation and ring deformation (see Sections 7 and 8). $\Delta E_{\mathrm{b}}^{\text {corr }}$ shows clear evidence for additional sources of energy increase.

\begin{tabular}{|c|c|c|c|c|c|c|}
\hline Year & Fill number & Energy & $t_{\mathrm{f}}$ & $\Delta t$ & $\Delta E_{\mathrm{b}}^{\text {raw }}(\mathrm{MeV})$ & $\Delta E_{\mathrm{b}}^{\text {corr }}(\mathrm{MeV})$ \\
\hline 1993 & 1636 & $\mathrm{P}-2$ & $2 \mathrm{~h}$ & $3.5 \mathrm{~h}$ & 6.1 & 1.3 \\
1993 & 1734 & $\mathrm{P}-2$ & $2 \mathrm{~h}$ & $4 \mathrm{~h}$ & 0.6 & 1.7 \\
1993 & 1772 & $\mathrm{P}-2$ & $1.5 \mathrm{~h}$ & $21 \mathrm{~h}$ & 6.1 & 7.5 \\
1993 & 1811 & $\mathrm{P}+2$ & $21 \mathrm{~h}$ & $5 \mathrm{~h}$ & 2.6 & -1.3 \\
1993 & 1849 & $\mathrm{P}-2$ & $13 \mathrm{~h}$ & $11 \mathrm{~h}$ & 4.0 & 3.1 \\
1993 & 1892 & $\mathrm{P}+2$ & $12 \mathrm{~h}$ & $2.5 \mathrm{~h}$ & 0.0 & 0.3 \\
1994 & 2234 & $\mathrm{P}$ & $12 \mathrm{~h}$ & $7 \mathrm{~h}$ & -1.0 & 0.6 \\
1994 & 2255 & $\mathrm{P}$ & $13 \mathrm{~h}$ & $6 \mathrm{~h}$ & 0.8 & 0.4 \\
1994 & 2395 & $\mathrm{P}$ & $21 \mathrm{~h}$ & $4 \mathrm{~h}$ & -3.3 & -0.6 \\
1994 & 2569 & $\mathrm{P}-2$ & $7 \mathrm{~h}$ & $8 \mathrm{~h}$ & 2.7 & 0.8 \\
1995 & 2929 & $\mathrm{P}-2$ & $4.5 \mathrm{~h}$ & $6 \mathrm{~h}$ & -1.3 & 1.5 \\
1995 & 3022 & $\mathrm{P}+2$ & $1.5 \mathrm{~h}$ & $16 \mathrm{~h}$ & 0.6 & 3.2 \\
1995 & 3029 & $\mathrm{P}+2$ & $2 \mathrm{~h}$ & $15 \mathrm{~h}$ & 5.7 & 3.1 \\
1995 & 3030 & $\mathrm{P}-2$ & $1 \mathrm{~h}$ & $21 \mathrm{~h}$ & 5.3 & 1.9 \\
1995 & 3036 & $\mathrm{P}+2$ & $1.5 \mathrm{~h}$ & $15 \mathrm{~h}$ & 3.2 & 2.4 \\
1995 & 3064 & $\mathrm{P}+2$ & $1 \mathrm{~h}$ & $6 \mathrm{~h}$ & 3.1 & 1.6 \\
\hline
\end{tabular}

as a function of time into a fill is shown in Fig. 3c. The field rise was also studied as a function of time of day: the rise was found to be much smaller in the 'quiet period' than in the remainder of the day. The rise as a function of time of day is shown in Fig. $3 \mathrm{~b}$.

Eventually the NMR noise was correlated with the measurement of a current flowing on the LEP beam-pipe. This current fluctuates in amplitude with time and position around the LEP ring, with a typical magnitude of about $1 \mathrm{~A}$. It enters close to the beam-injection lines at IP1 and flows in both directions around LEP, exiting near the Versoix river at IP6 (Fig. 4). The current's behaviour in the regions of NMR4 and NMR8 is consistent with the anticorrelated noise seen in these devices.

These observations led to the definition of the pseudo-device NMR48, where

$$
\mathrm{NMR} 48 \equiv \frac{5}{8} \mathrm{NMR} 4+\frac{3}{8} \mathrm{NMR} 8
$$

yields an 'average NMR' sampling the field at both positions around the ring, eliminating almost completely the anticorrelated noise.

The choice of coefficients in Eq. (7), defining NMR48, was based on the assumption that each NMR represents the average behaviour of, respectively, the three or five octants between IP1 and IP6. This assumption is confirmed by the detailed measurements of the amplitude of the parasitic current flowing along the beam-pipe.

The current arises from electrical trains passing along the Geneva-Bellegarde railway line [13]. A fraction of the direct current powering the trains leaks to Earth when returning to the generator station, and this leakage current passes through the Earth and on to the LEP beam-pipe. The quiet period between midnight and 05:00 is a consequence of there being no trains running in the area at this time. A dedicated experiment monitoring the voltage between the train rails and Earth recorded a signal clearly 


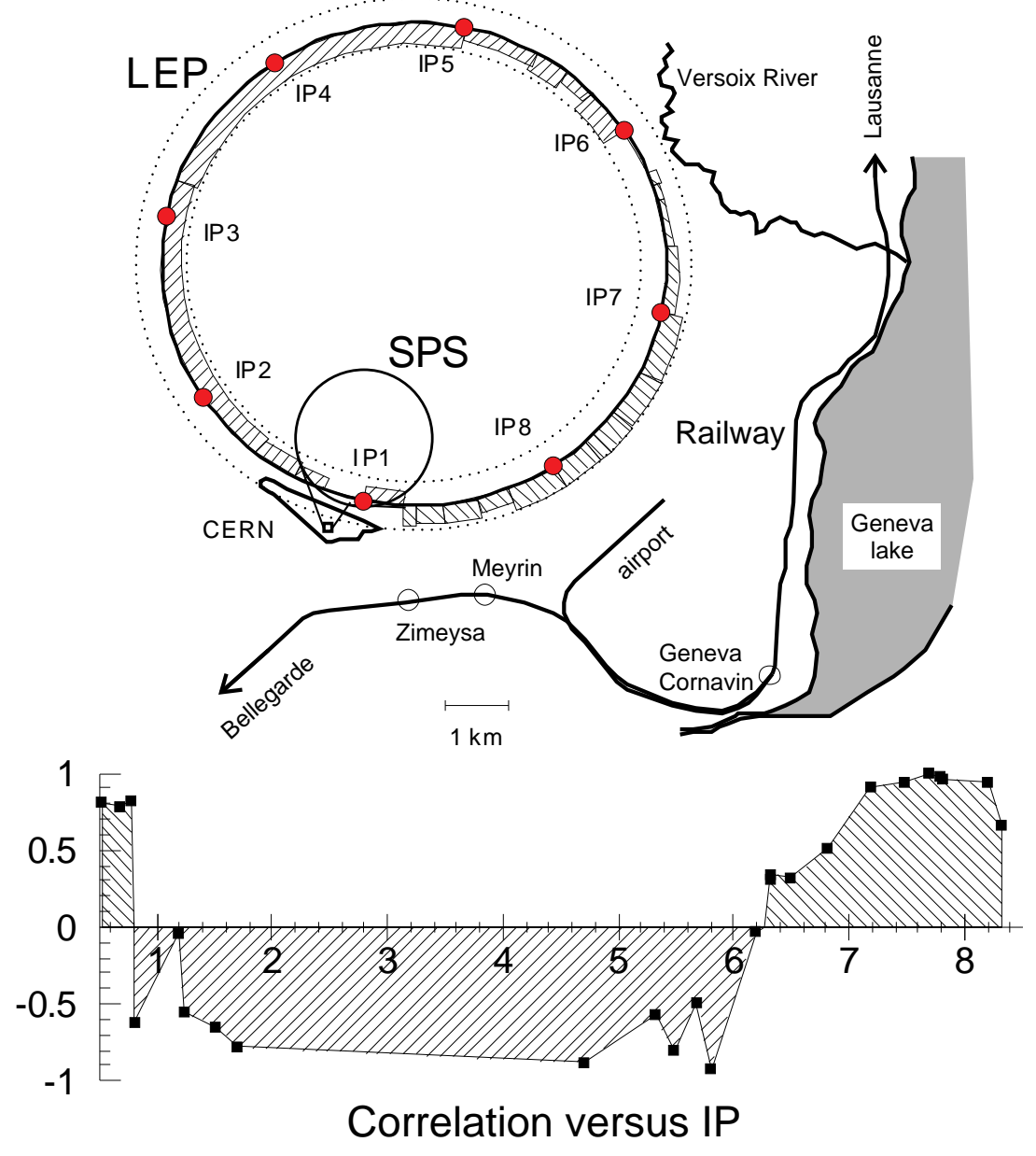

Figure 4: Diagram of CERN accelerators and surroundings. The measured correlation pattern of the parasitic currents along the ring is shown, proving that they enter and leave LEP near IP6 and IP1. 


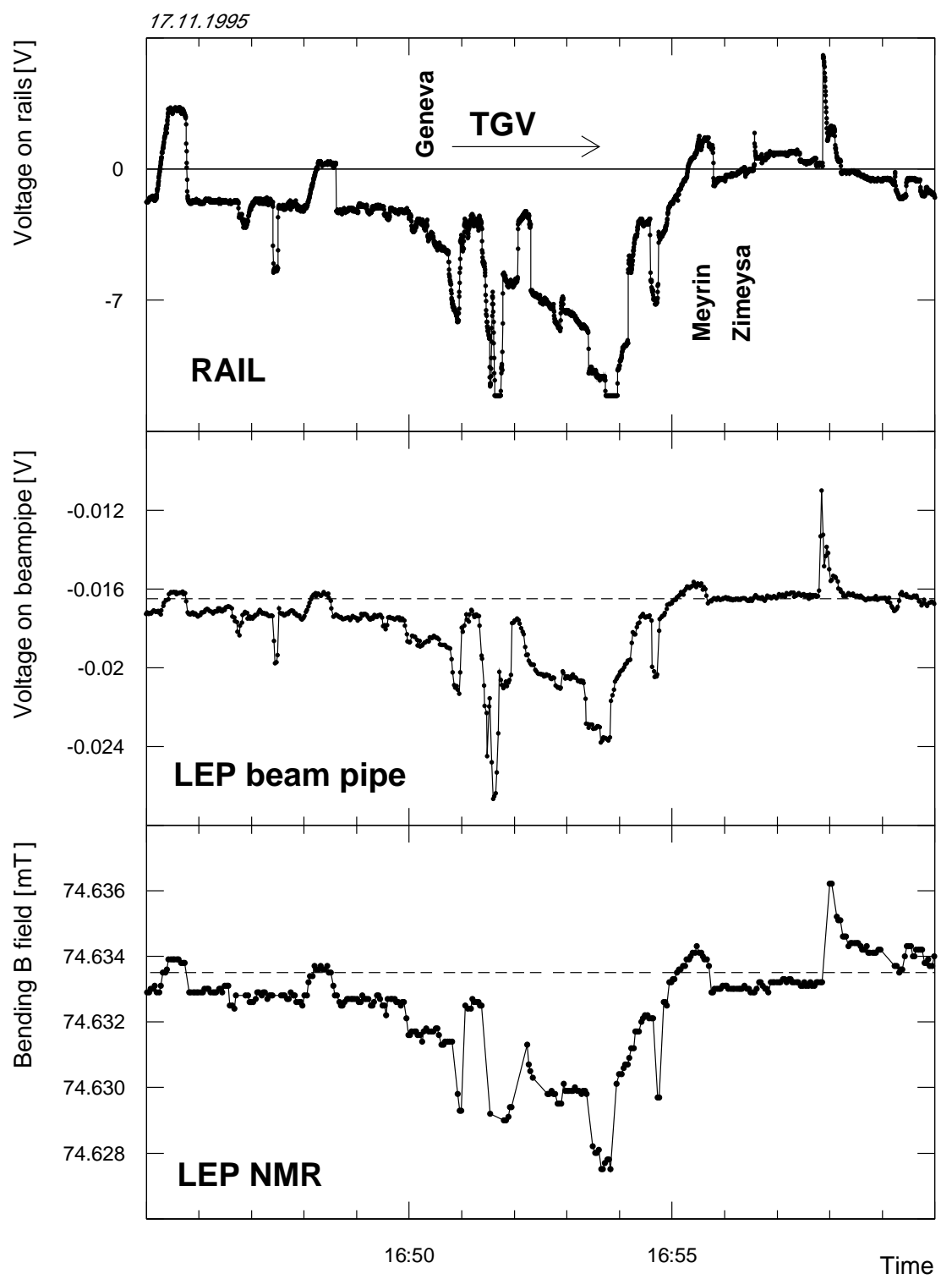

Figure 5: The synchronous measurement of the voltage difference between ground and the train rails (top), the voltage difference between the LEP beam pipe and ground (middle) and the NMR readings (bottom). The correlation is obvious. The label 'Geneva' marks the time of the departure of the TGV ('Train à Grande Vitesse') from Geneva central station. The label 'Zimeysa' indicates the time when the TGV went past the measuring device. 


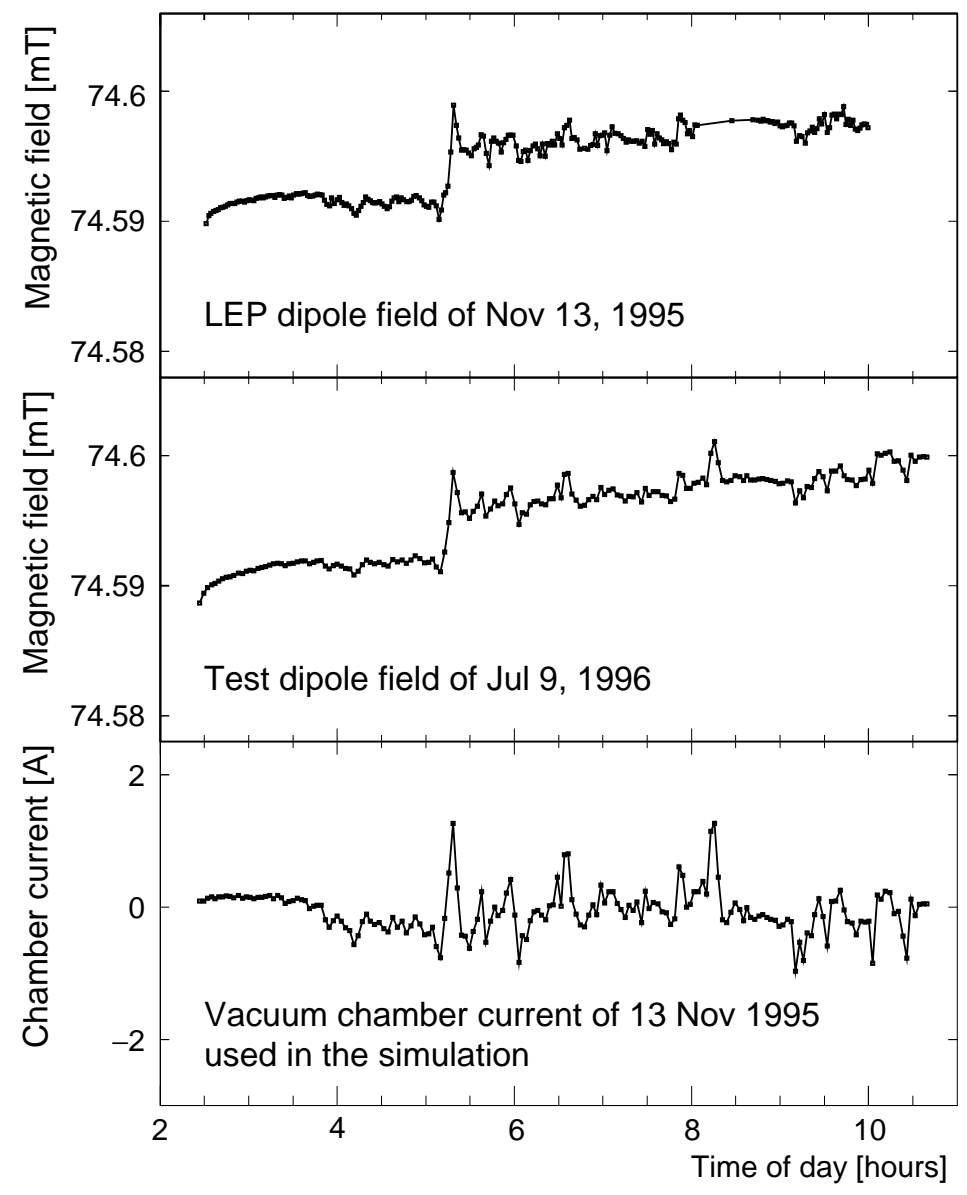

Figure 6: The NMR recording from one of the LEP dipoles (top figure) is compared with the NMR readings from the test dipole in the laboratory (middle figure) when the parasitic current recorded from the LEP beam-pipe (bottom figure) is 'played back' on the section of beam-pipe inserted in the laboratory dipole.

correlated with the variations in the beam-pipe current and the NMR field (see Fig. 5).

The currents flowing on the beam-pipe perturb the magnetization status of the iron. The tendency to saturate apparent in Fig. 3d is due to the finite amplitude of the current spikes. The level of saturation depends on the combined effect of temperature and current peak patterns (see Fig. 6 and Section 5).

For the 1996 high energy running, in addition to NMR4 and NMR8, probes were installed in the other octants to provide additional sampling of the magnets around the ring. Two octants were instrumented with five probes each, giving a total of 16 NMRs. The data from these devices enabled a cross-check of the behaviour observed in 1995 to be made with a larger sample of magnets. In comparing the data of the two years, certain differences in operating conditions had to be taken into account. In particular, in 1996 the magnets were run at approximately twice the field and had a more significant temperature excursion. In addition, no bending modulation was performed prior to the start of a fill.

The 1996 NMR data indicated a field rise in all octants. The three octants between IP1 and IP6 behaved in a similar manner to that seen in NMR8, with sudden steps in field, while the smoother field evolution in the remaining five octants was similar to that seen by NMR4 (see Fig. 7). The short-term noise was anticorrelated around the ring in a manner expected from the measurements of the current on the beam-pipe as shown in Fig. 4. This supports the choice of coefficients in Eq. (7). The variation of the short-term noise and of the field rise itself with time of day and time into fill confirmed that observed in 1995. The rise averaged over all the octants was compatible with that seen in the weighted mean of octants 4 and 8 . This demonstrates that the pseudo-device NMR48 is representative of the LEP ring as a whole. Further discussion of these phenomena can be found in [8], [13] and [14]. 

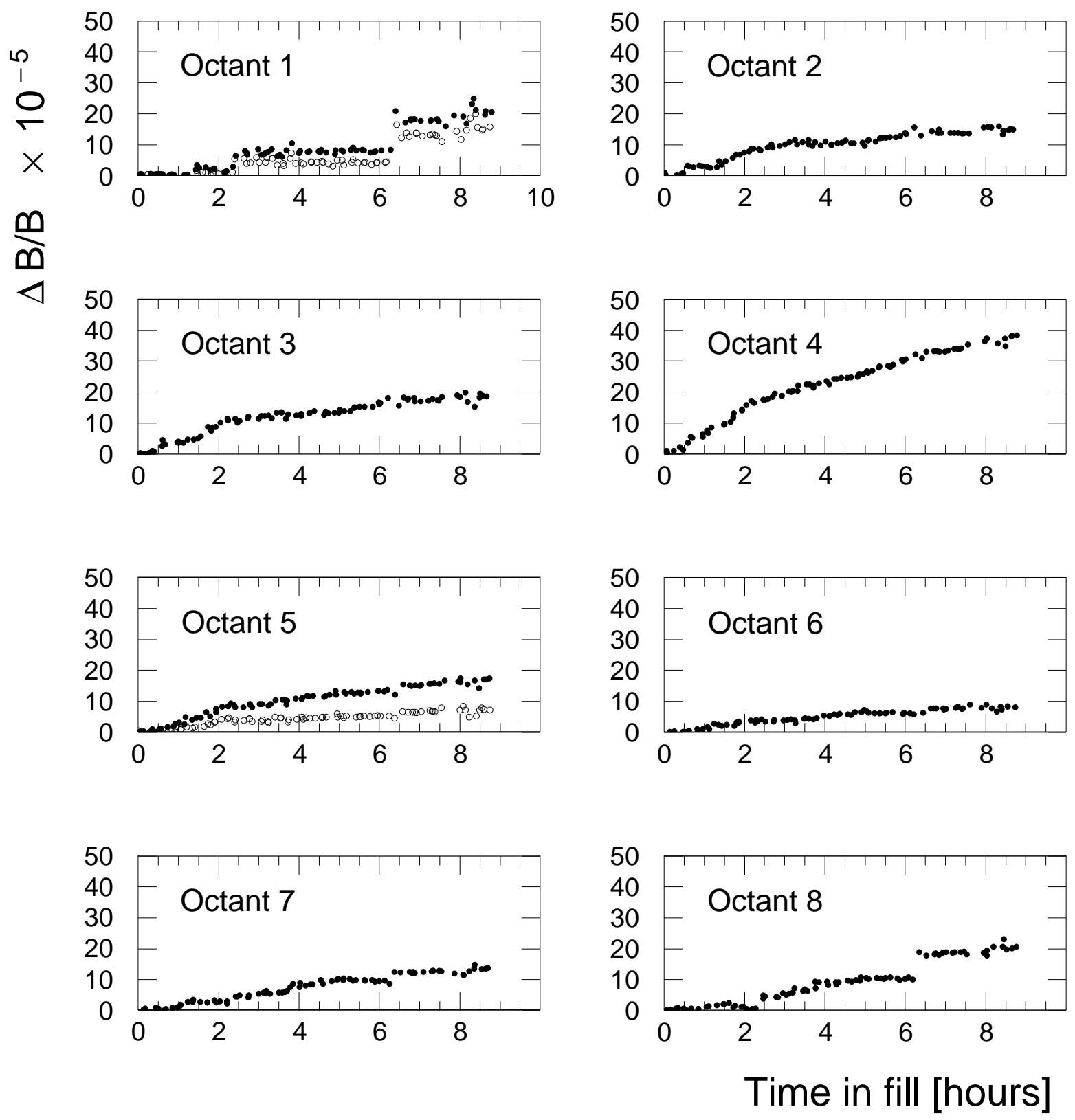

Figure 7: The fractional field rise observed by the NMR probes in each of the LEP octants for a typical fill in 1996 at a beam-energy of $86 \mathrm{GeV}$. Octants 1 and 5 are equipped with five probes, of which the two exhibiting the minimum and maximum rise are displayed. Each dipole has experienced a different temperature excursion; for instance the temperature rise for the instrumented magnet in octant 4 is three times that for octant 6 . The nonlinear behaviour caused by parasitic currents on the beam-pipe is evident in octants 1,7 and 8 . 

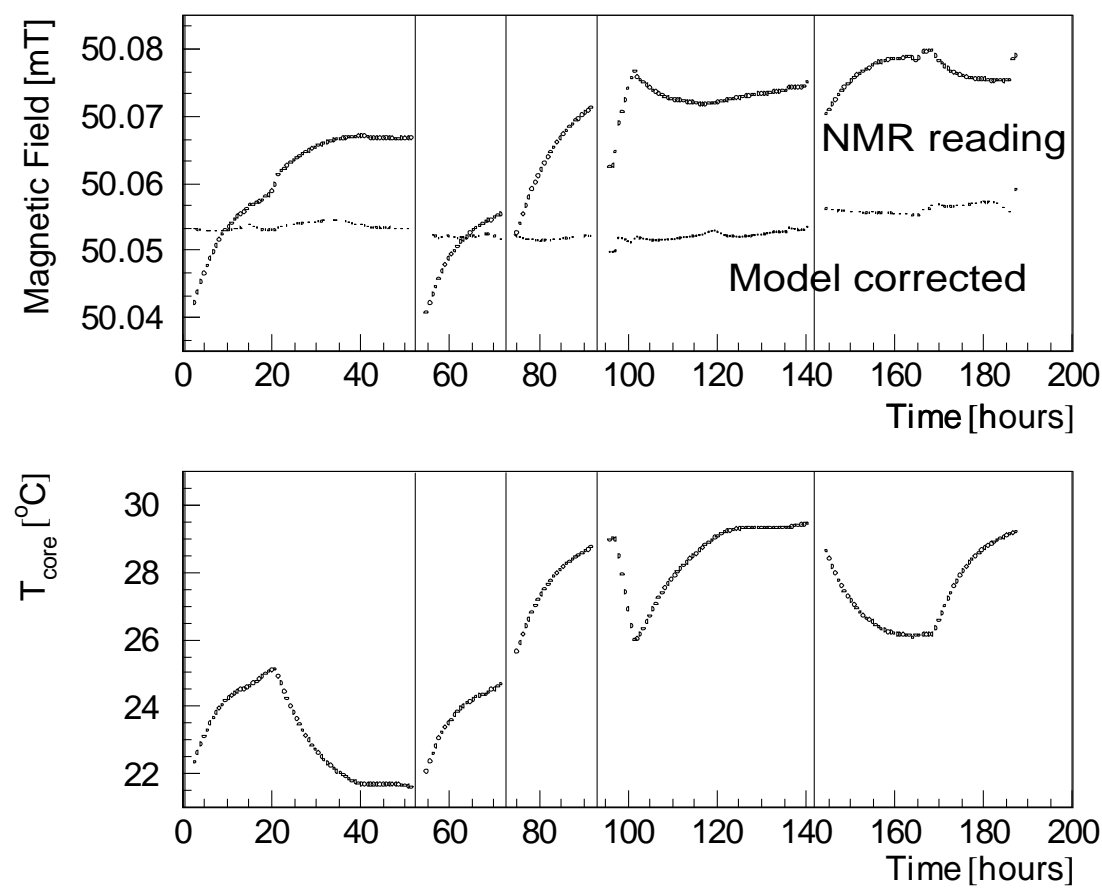

Figure 8: Temperature experiment performed at the ISR test facility. The two lines in the top figure represent the raw magnetic field and that which is obtained after subtraction of the contribution due to temperature effects. The lower figure shows the mean temperature of the magnet. Vertical bars mark each demagnetization cycle.

\section{The temperature behaviour of a LEP dipole}

The thermal behaviour of the LEP magnet cores is driven by several heat sources, sinks and the resulting temperature gradients. The major power sources are resistive-loss heating of the excitation bars, synchrotron radiation on the beam-pipe and the cooling water which circulates along both the beam-pipe and the excitation bars. The power transfer to the air happens mostly by radiation exchange as the small temperature difference between the air and the magnets is not sufficient to drive a strong convective motion.

The temperature variations induced by all of these effects produce distortions of the geometry of the LEP dipoles.

- The C-shaped dipole cores (see Fig. 1), and thus the gap between the poles, tend to expand due to the average temperature variation and to open or close depending on the temperature gradients around the magnet excitation bars.

- The thermal expansion of material leads to variation of the pole area.

- Thermally induced stresses arise at the contact interface between the soft iron slabs and the concrete. These result in a change of pressure propagating to the centre of the slabs which modifies the relative permeability coefficient of the iron [15] and introduces thermal hysteresis effects in the field. This is the dominant thermal effect influencing the magnetic field.

From the test laboratory (see Section 2.11) the dependence of the field on measurable quantities such as the core and cooling-water temperature could be derived experimentally.

\subsection{Experimental results}

Figure 8 shows the result of a particular experiment used to determine the thermal model for the LEP dipoles. This experiment, which lasted over eight days, consisted of five periods. Each of these starts with a demagnetization cycle, described in Section 2.5. The current is then set to $2000 \mathrm{~A}$, which is the value used to run LEP at the $\mathrm{Z}$ peak energy.

In the following period (varying from 10 to $40 \mathrm{~h}$ ) the temperature of the cooling water is changed 
in a controlled way. The core temperature follows these changes with a time constant of $5.5 \mathrm{~h}$. Unexpectedly the first period shows a monotonic rise of the field for both increasing and decreasing core temperature. The second and the third are partial repetitions. The fourth and the fifth show both increase and decrease of the magnetic field by applying the reverse temperature variation pattern with respect to the first.

The agreement between experiments sharing the same conditions and history is quite good, while applying a different history or simply reversing the time order of the temperature variations produces different results.

The model used previously [4], where the field changes were simply proportional to the temperature variations $\left(\Delta B=C_{\mathrm{T}} \cdot \Delta T\right)$, cannot reproduce these observations.

\subsection{The thermal model}

The model developed to describe the thermal behaviour of LEP dipole magnets includes five temperature parameters:

$$
\begin{aligned}
B= & B_{0} \cdot\left[1+C_{\mathrm{T}_{\mathrm{i}}} \cdot\left(T_{\mathrm{i}}-T_{0}\right)+C_{\mathrm{T}} \cdot\left(T-T_{\mathrm{i}}\right)\right. \\
& +C_{\mathrm{T}_{\mathrm{GRAD}}} \cdot\left(T-T_{\mathrm{W}}\right)+C_{\mathrm{T}_{\mathrm{MAX}}} \cdot\left(T_{\mathrm{MAX}}-T_{\mathrm{i}}\right) \\
& \left.+C_{\mathrm{T}_{\mathrm{MIN}}} \cdot\left(T_{\mathrm{MIN}}-T_{\mathrm{i}}\right)\right]
\end{aligned}
$$

where $T_{0}$ is the reference temperature, $T_{\mathrm{i}}$ is the core temperature at the beginning of the period, $T$ is the dipole core temperature, $T_{\mathrm{W}}$ is the cooling-water temperature, $T_{\mathrm{MAX}}$ and $T_{\mathrm{MIN}}$ are respectively the maximum and minimum core temperatures since the beginning of the period, $B$ is the corrected magnetic field and $B_{0}$ is the magnetic field at $T_{\mathrm{i}}, T, T_{\mathrm{W}}, T_{\mathrm{MAX}}, T_{\mathrm{MIN}}=T_{0}$.

From experience accumulated at the test facility, the phenomenological description of the parameters is as follows:

- $C_{\mathrm{T}_{\mathrm{i}}}$ : this term accounts for changes in the geometry of the magnets as well as stresses in the iron.

- $C_{\mathrm{T}}$ : this term arises mainly from bulk change of size of the cores without stress variation in the interface region.

- $C_{\mathrm{T}_{\mathrm{GRAD}}}$ : the temperature gradient across the section of the magnet changes the size of the air gap. The temperature difference between the core and the cooling-water is taken as an estimate of this gradient since the thermal conductivity of the aluminium (coil) is much larger than that of the concrete.

- $C_{\mathrm{T}_{\mathrm{MAX}}}, C_{\mathrm{T}_{\mathrm{MIN}}}$ : the underlying explanation for these parameters is not fully understood. Large variation of the stress status in the interface region seems to be the main cause.

The values of the five coefficients measured for the test magnet were:

$$
\begin{aligned}
C_{\mathrm{T}_{\mathrm{i}}} & =(6.4 \pm 0.6) \cdot 10^{-5}{ }^{\circ} \mathrm{C}^{-1} \\
C_{\mathrm{T}} & =(-3.3 \pm 0.3) \cdot 10^{-5}{ }^{\circ} \mathrm{C}^{-1} \\
C_{\mathrm{T}_{\mathrm{GRAD}}} & =(0.4 \pm 0.04) \cdot 10^{-5}{ }^{\circ} \mathrm{C}^{-1} \\
C_{\mathrm{T}} & =(12.0 \pm 1.2) \cdot 10^{-5}{ }^{\circ} \mathrm{C}^{-1} \\
C_{\mathrm{T}_{\mathrm{MIN}}} & =(-4.2 \pm 0.4) \cdot 10^{-5}{ }^{\circ} \mathrm{C}^{-1}
\end{aligned}
$$

The most important parameter is $C_{\mathrm{T}_{\mathrm{MAx}}}$ since it is the largest one and $T_{\mathrm{MAX}}$ has the greatest variation during LEP physics fills. The $10 \%$ relative error on the determination of these parameters is assigned based on the quality of the fits and the reproducibility of the experimental determinations. The uncertainties on these parameters for the well known and controlled test magnet are, in any case, smaller than the final error on the same parameters for LEP due to the spread of characteristics of the 3336 LEP dipoles.

\subsection{Application to the LEP environment}

The cooling system of LEP is operated in such a way that the gradient term $C_{\mathrm{T}_{\mathrm{GRAD}}} \cdot\left(T-T_{\mathrm{W}}\right)$ is always negligible. The term $C_{\mathrm{T}_{\mathrm{i}}} \cdot\left(T_{\mathrm{i}}-T_{0}\right)$ is constant within a fill by definition since $T_{\mathrm{i}}$ can vary only 

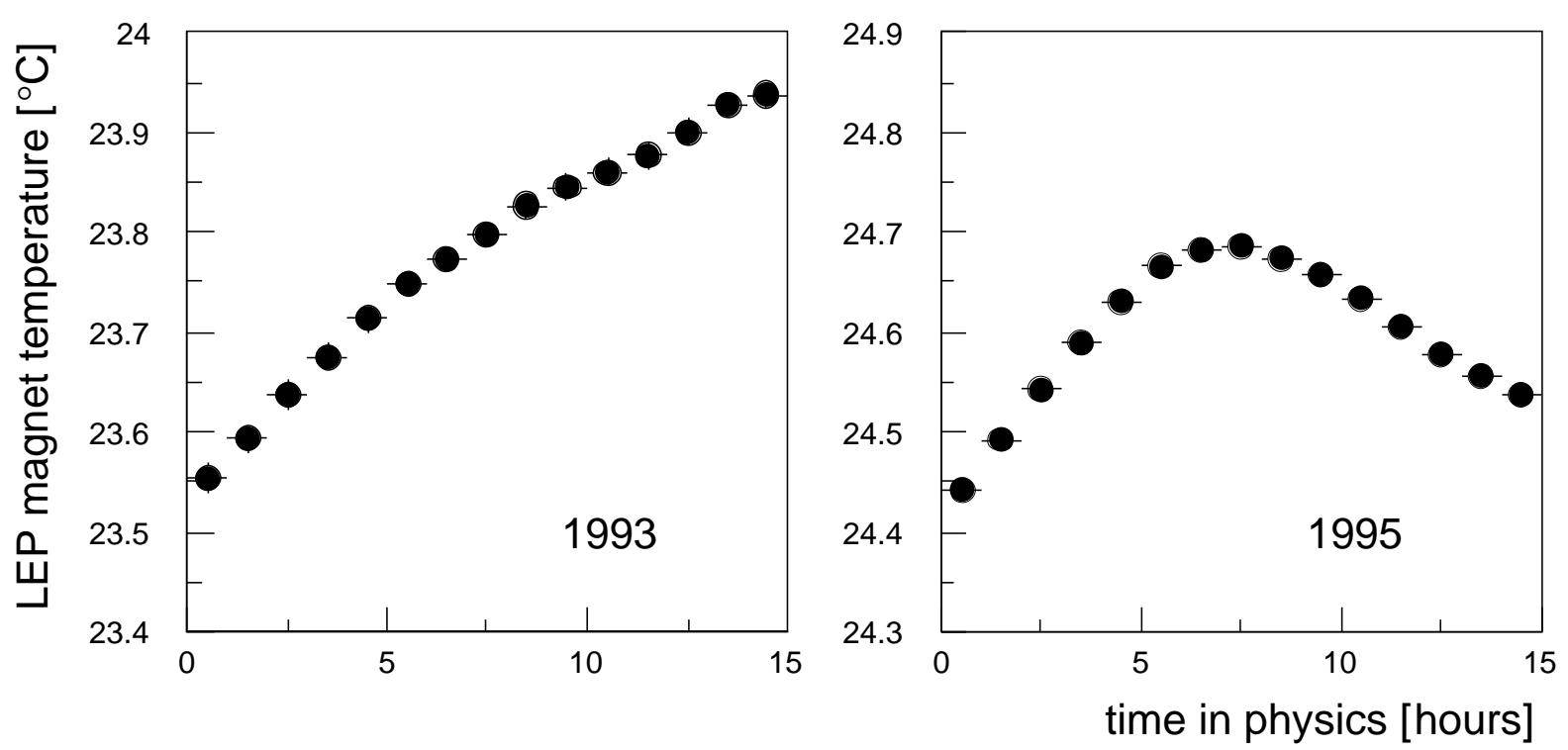

Figure 9: Average temperature profiles during 1993 and 1995 LEP physics fills. For 1994 the temperature profile is the same as in 1993.

from fill to fill: most of its effect is absorbed into the overall offset for a fill ( $E_{\text {norm }}$ in Eq. 5) and for simplicity this term was ignored. Checks were performed by including this term in the model: the largest observed change in the mean energy of any energy point was $0.03 \mathrm{MeV}$, and is thus negligible.

The typical temperature profile for the 1993 and 1994 physics fills is monotonically increasing (Fig.9, left). In this case the $C_{\mathrm{T}_{\mathrm{MIN}}}$ term is always zero and the use of a single parameter equal to $C_{\mathrm{T}_{\mathrm{MAX}}}+C_{\mathrm{T}}$ is sufficient to parametrize the field variation, proportional to $T-T_{\mathrm{i}}$.

In 1995 the situation was different, since the cooling regulation was changed to reduce temperature excursions in the magnets (see Section 2.6). In this case the profile was of rising temperature for the first few hours and then a partial decrease (Fig. 9, right). Even in 1995 the temperature of the magnets rarely decreased below the initial temperature, $T_{\mathrm{i}}$, making the $C_{\mathrm{T}_{\mathrm{MIN}}}$ term irrelevant.

The model can then be simplified and reduced to only two parameters, one to be used during increases and one during decreases of the mean temperature of the magnet cores.

$$
\frac{1}{B_{0}} \frac{d B}{d T}= \begin{cases}C_{\mathrm{T}_{\mathrm{MAX}}}+C_{\mathrm{T}} \equiv C_{\mathrm{eff}} & \text { if } \frac{d T}{d t}>0 \\ C_{\mathrm{T}} & \text { if } \frac{d T}{d t}<0\end{cases}
$$

Since $C_{\text {eff }}>0$. and $C_{\mathrm{T}}<0$., the application of this model to all periods of LEP operation results in a monotonic increase of the magnetic field.

\subsubsection{Interplay between temperature and parasitic-current effects}

Because both temperature changes and parasitic currents on the beam-pipe (see Section 4) generate field rises, it is difficult to disentangle the two contributions. Moreover, the way these two effects combine is not straightforward: studies performed at the test facility have shown that the two sources of drift compete to determine the saturation level of the dipole field. The dipole-field rise is described as a superposition of the effect of temperature and of the instantaneous current flowing on the beampipe. Any current spike has an effect only if, when added to the underlying drift due to temperature, its amplitude exceeds that of any of the preceding spikes. From the laboratory measurements it is seen that only approximately $65 \%$ of the instantaneous field due to these spikes is left as permanent magnetization of the dipole. A given field can be reached either by a temperature variation or by means of a current spike, whichever comes first. For example, when a large current spike causes a jump in the field, the field will stay constant until either the temperature-induced variations reach an equivalent level or a larger spike happens. The saturation effects are explained both by the stabilization of the dipole temperatures and by the finite amplitudes of the current spikes. In fact, the random time distribution of the largest 


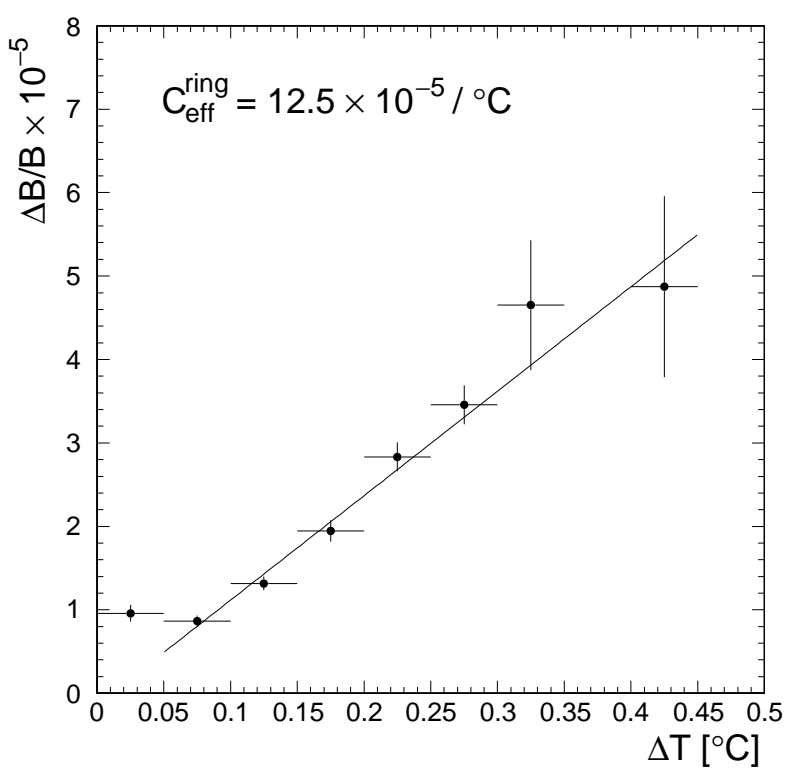

(a) $C_{\text {eff }}^{\text {ring for ring }}$

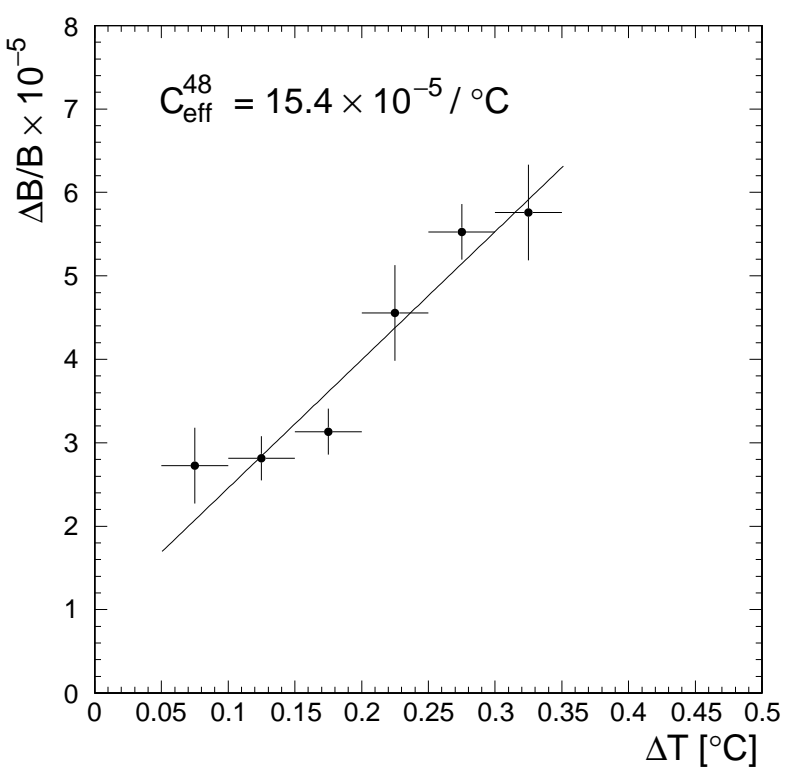

(b) $C_{\text {eff }}^{48}$ for NMR48

Figure 10: Example fits to the coefficient $C_{\mathrm{eff}}^{\text {ring }}$ for the ring and $C_{\mathrm{eff}}^{48}$ for NMR48. These data are restricted to the period 00:00 $<t_{\text {day }}<$ 05:00, and yield the fits with the maximum deviations from the estimated central values for these coefficients.

current spike explains, at least qualitatively, the variation on a fill-by-fill basis of the time evolution of the field drifts. The magnet is reset only after a demagnetization cycle. An empirical model including these features has been developed which predicts quite well the field variations [16]. The model demands the knowledge of the current flowing on the beam-pipe. As this input parameter was not generally available, an approximated version has been developed to cross check the LEP field model used to deliver the experimental energies (see Section 6.2).

\subsection{Determination of LEP temperature coefficients}

The parameters shown in Eq. (9) have been determined from the LEP dipoles themselves using the data of 1996. The 16 dipoles equipped with NMR and temperature probes enabled a better sampling of the average ring behaviour than the two dipoles, NMR4 and NMR8, which had been equipped in this manner during 1995. Furthermore, the large temperature excursions of about $1^{\circ} \mathrm{C}$ during the high-energy running of 1996 produced a suitable data set to study the temperature behaviour of the magnets. This is to be compared with 1995 , where the short-term temperature variations were only about $0.2^{\circ} \mathrm{C}$ and similar to the precision of the measurements. In the determination of $C_{\text {eff }}$ of Eq. (9) all high-energy physics running in 1996 was analysed.

Successive recordings at intervals of six minutes were considered, and the relative field change $\Delta B / B$ was plotted against the absolute temperature change $\Delta T$. This was done for the first eight hours of fills, during which the temperature was continuing to rise. Intervals with large field jumps, presumably induced by stimulation from parasitic currents, were discarded.

A linear fit was made in $\Delta B / B$ against $\Delta T$ for the region $\Delta T>0$. This fit was made both for the mean behaviour of all the instrumented dipoles, to represent the ring, and for the weighted mean of NMR4 and NMR8 using the coefficients of Eq. (7), to represent the pseudo-device NMR48 used in the energy-rise modelling. To assess systematic stability, the analysis was restricted to the period $0<t_{\text {day }}<5$ hours, the definition of 'large field jump' was varied and the fit range and the bin width were changed. In addition, complementary analyses were performed where the temperature coefficient was fitted simultaneously with terms representing independent field variation caused by the beam-pipe current (Section 5.4.1).

The results of the various fits showed a significant variation, indicating the presence of systematic 

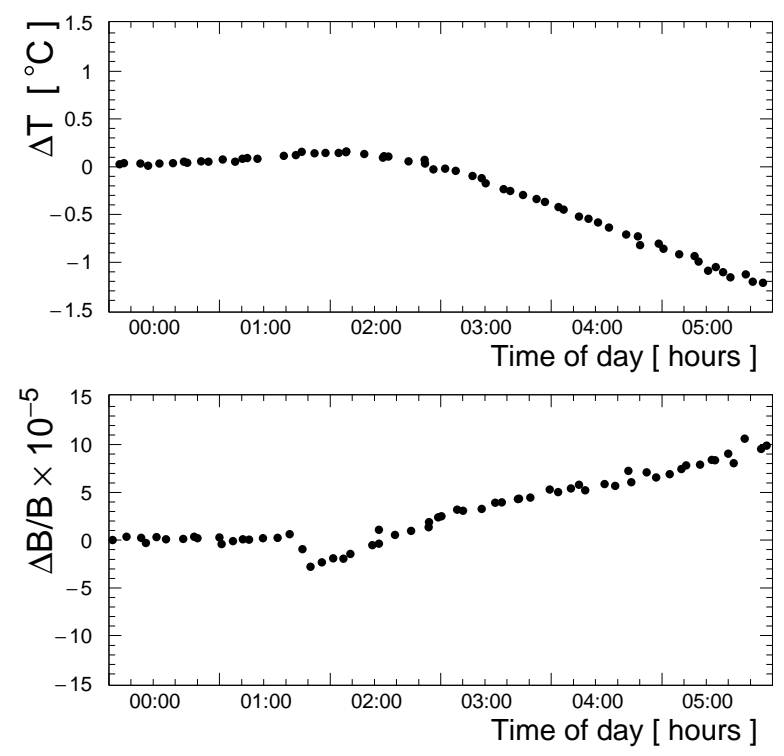

Figure 11: Variation in temperature and dipole field during the temperature experiment, fill 3822. Both quantities are shown relative to their value at midnight, and are averaged over all instrumented dipoles. The dip at $1.6 \mathrm{~h}$ coincides with the abrupt turn off of the LEP quadrupoles, done in order to speed the cooling of the refrigeration water. This manipulation is never done during physics conditions.

effects and the difficulty of separating the parasitic current and temperature-driven behaviour. An effective coefficient $C_{\text {eff }}$, of $10 \cdot 10^{-5} /{ }^{\circ} \mathrm{C}$ was found to describe both the ring and NMR48 data, with uncertainties of $\pm 30 \%$ and $\pm 60 \%$ respectively. Example fits to $C_{\mathrm{eff}}^{\text {ring }}$ and $C_{\mathrm{eff}}^{48}$ for the ring and NMR48, are shown in Figs 10a and b respectively. The NMR jumps are bigger and more frequent in octant 8 than elsewhere in the ring, leading to the larger error for $C_{\mathrm{eff}}^{48}$.

To determine the effective coefficient $C_{\mathrm{T}}$ for periods in which the dipole temperature decreases, a dedicated experiment was performed during fill 3822 in 1996 . Without beam in the machine the dipoles were ramped to $45 \mathrm{GeV}$ with the cooling system inhibited. This provoked a steady rise in core temperature. At about 02:00 the cooling was re-enabled, and a decrease in core temperature of about $1^{\circ} \mathrm{C}$ was observed in the next three hours. As expected from the laboratory temperature model, the temperature decrease was accompanied by a rise in the field of the dipoles, as monitored by the NMR devices. The relative change in the temperature and dipole field with respect to the reading at midnight is shown in Fig. 11.

Because the field rise occurred during the period in which noise currents are at a minimum, it can be attributed directly to the change in temperature. In extracting the temperature coefficient $C_{\mathrm{T}}$ from these data, the full temperature model must be invoked, taking account of the terms involving $C_{\mathrm{T}_{\mathrm{GRAD}}}$ and $C_{\mathrm{T}_{\mathrm{MIN}}}$ in expression (8), as the conditions of the experiment are sufficiently extreme as to render these effects significant. The values of $C_{\mathrm{T}_{\mathrm{GRAD}}}$ and $C_{\mathrm{T}_{\mathrm{MIN}}}$ were fixed to those measured for the laboratory dipole, and $C_{\mathrm{T}}$ determined for each instrumented dipole. The results obtained were similar in magnitude to the coefficients found for the laboratory dipole. A value of $C_{\mathrm{T}}=(-5.0 \pm 2.5) \cdot 10^{-5} /{ }^{\circ} \mathrm{C}$ was adopted, for both the ring as a whole and the pseudo-device NMR48. The error covers the variation seen between the dipoles and also accounts for the systematic uncertainties in subtracting the other terms in the temperature model.

\subsubsection{Additional checks on the BOF-fills}

The six BOF-fills in 1995, together with the long-term calibration machine development measurements made in 1993 and 1994, allow various cross-checks to be performed on the parameters of the model for the LEP energy. These data are sensitive to the tide-model parameters (see Section 7.1), the temperature coefficient of the ring and to variations during a fill from other effects.

A model was used to make a fit to these RD data which took into account the variation due to tides, the ring temperature coefficient, $C_{\text {eff }}^{\text {ring }}$, and a functional form for the rise during a fill as a function of time of day and time into fill. For the fills used in the fit the temperature was almost always 
increasing, so that there is no sensitivity to the coefficient $C_{\mathrm{T}}$ in Eq. 9. The value for $C_{\text {eff }}^{\text {ring }}$ is found to be $(10 \pm 2) \cdot 10^{-5} /{ }^{\circ} \mathrm{C}$, where the error is the statistical uncertainty from the fit. This is in good agreement with that determined from the 1996 data.

\section{$6 \quad$ Modelling the energy rise in the fill}

Since more than half of the data at the $\mathrm{Z}$ resonance were collected prior to 1995, the model for the dipole field drifts must be independent of the direct NMR readings in order that it can be applied to the 1993 and 1994 datasets. The dipole drifts depend on the temperature changes of the dipole cores and on the parasitic currents flowing on the beam-pipe, which are related to the railway traffic and depend on the time of day. Moreover, the way the magnets react to these stimulations depends on the time elapsed since the last cycling of the field, the time into the fill.

The model chosen is based on straightforward two-dimensional binning against time of day and time into fill of the available NMR data after subtraction of the temperature effects (phenomenological model). Other treatments of these data were explored in order to gauge the systematic stability of this method. In addition, another (deterministic) model has been developed based on the experiments with the dipole in the ISR facility and the recording of beam-pipe currents in a typical week.

The phenomenological model treats the temperature and current effects independently; the comparison with the deterministic model allows a cross-check of this assumption. Both models necessarily assume the daily behaviour of the parasitic currents to be essentially the same throughout all LEP operation.

\subsection{Model based on NMR readings (phenomenological)}

The model used for the determination of the central values is based on the assumption that the NMR48 readings are representative of the magnetic behaviour of the whole of the ring. The data collected in 1996 from additional NMR probes have shown this to be a good approximation.

The model takes the rise seen in the combination of the two devices, subtracts the effect of temperature and parametrizes the resulting rise as a function of two parameters: the time of day, and the elapsed time in the fill. The temperature model used to subtract the effect of the temperature is the one described in Section 5. When this is done the residual rise, attributable to parasitic currents, is about half that of the total rise as can be seen in Fig. 3d. The time of day correlation comes from the train schedules, whereas the elapsed time in the fill determines the saturation behaviour seen in the energy rise. No assumption on the functional form for the two rises is made. The NMR data, after rudimentary quality cuts (both devices working; no bending modulation happening at that instant), are simply represented in a two-dimensional histogram with 10 time-into-fill bins, of two hours each, and 5 time-of-day bins (one bin between midnight and 05:00 and four equal bins for the rest of the day). The mean value of each bin is then used to obtain the energy correction at any specific instant.

The model implicitly assumes that the effects of temperature and noise currents can be added linearly. This model, developed from the 1995 data, can then be applied to the 1993 and 1994 data with no modifications, under the assumption that the average effects of the parasitic currents are the same over the years. The only difference is the assumed beam-energy at the start of a fill.

\subsubsection{Effect of bending modulations}

Since bending modulations have an important effect on the subsequent dipole energy rise, their consequence is taken into account by treating all data taken before (i.e. without) and after a bending modulation as separate samples, and filling a histogram for each as a function of time into fill. In the model a bending modulation causes a rise of $2.5 \pm 1.0 \mathrm{MeV}$ where this value was derived from all the available NMR data.

\subsubsection{Beam-energy at the start of a fill}

For fills calibrated at EOF, the initial energy is fixed by extrapolating backwards in time from the RD measurement using the model. For uncalibrated fills a nominal value is used which is normalized to the mean of the calibrated fills. 


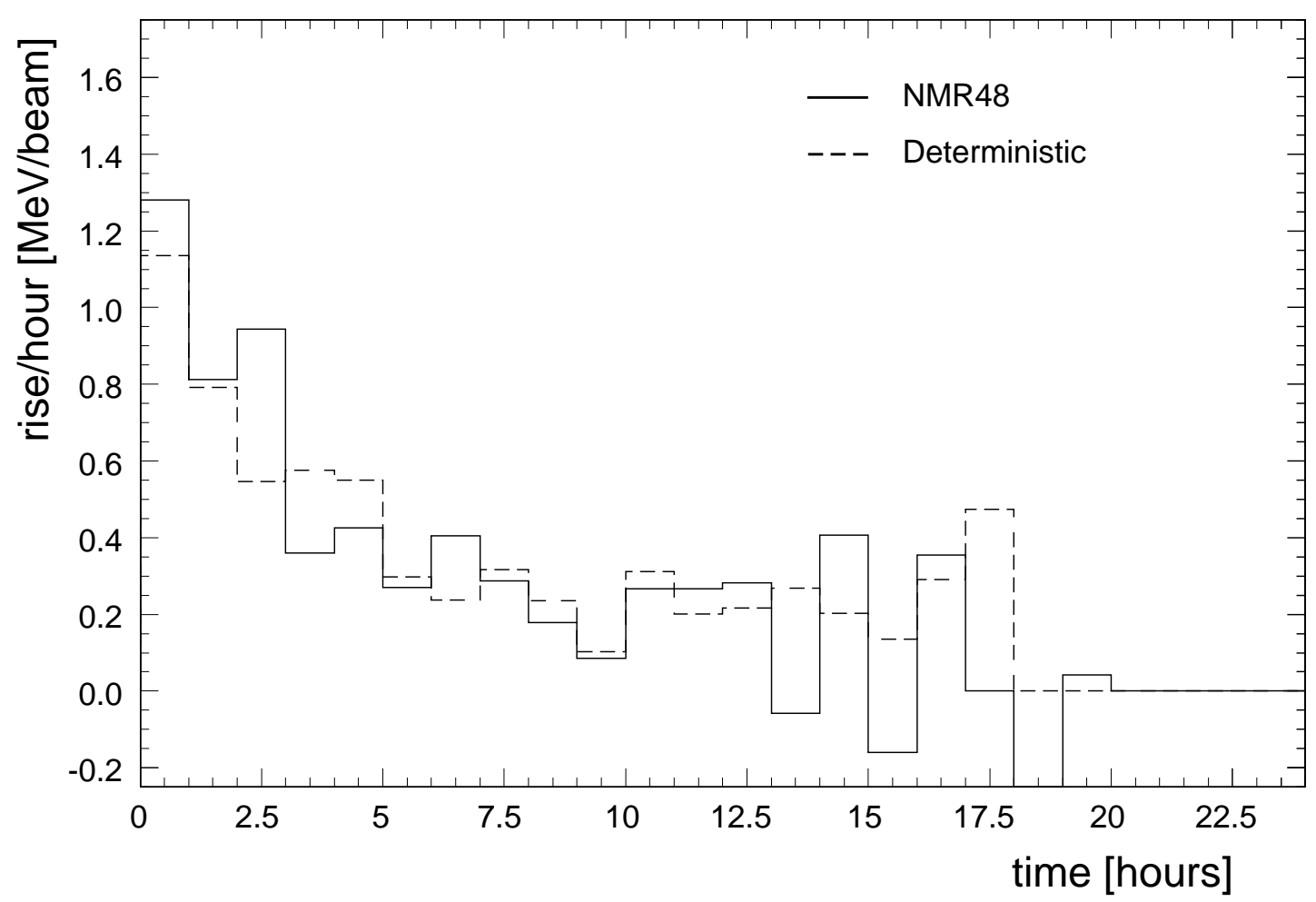

Figure 12: Average rise per hour as a function of time since the beginning of the fill. The full line represents the recorded NMR48 values averaged over the LEP runs without bending modulation in 1995. The dotted line represents the prediction for the same quantity by the deterministic model.

This nominal value is assumed to be the same for all fills at the same energy point in 1995 . During 1994 a drifting dipole-magnet power supply does not permit the same treatment: the energy is taken to follow the dipole supply current variations during the year. For 1993, due to the frequent fluxloop calibrations which have an important effect on the demagnetization of the dipoles, the starting field of the reference magnet was used instead.

\subsection{Model based on parasitic currents (deterministic)}

It has been demonstrated in the laboratory that the dipole field rise is a function of two quantities: the parasitic currents flowing on the LEP beam-pipe, and the temperature evolution of the magnet [16]. The reproducibility of the field rise is very good. Replaying the current recorded on the actual LEP beampipe and following a similar temperature history, a test magnet can accurately reproduce the rise seen in one of the tunnel magnets equipped with an NMR (see Fig. 6). The deterministic model estimates the beam-energy changes directly from these quantities (see Section 5.3.1 for a description of the interplay between temperature and current-spike effects).

To use such a model in practice the LEP ring is subdivided into eight octants and it is assumed that within an octant all currents and temperatures are the same. Another input is the temperature profile per octant, available for every year, and the current flowing on the beam-pipe per octant. The latter was recorded for a week during 1995 close to IP 5 , while the noise current correlation factors between octants were also measured [8] (see Fig. 4).

In Fig. 12 the average rise per hour during a fill, as measured by the NMR48 pseudo-device and the prediction of the model, are shown. The influence of the small variation of the train schedule from day to day has been computed and found to be small. The conversion factor between parasitic current 


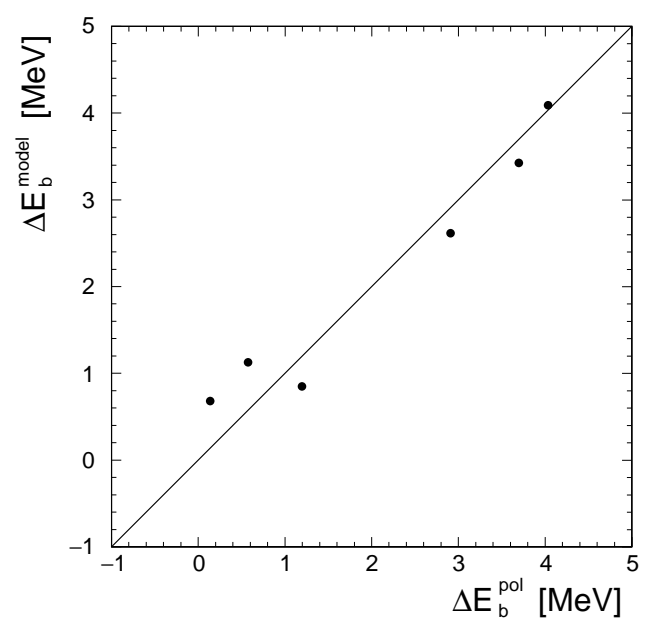

Figure 13: The estimate of the beam-energy rise from the phenomenological model versus the actual one for the six BOF fills of 1995. The rms of the difference is $0.4 \mathrm{MeV}$. Temperature effects have been subtracted.

and dipole field is obtained by tuning the model on the NMR data so that the measured energy rise is obtained. The model can be used on all years and NMR information is used only for the normalization.

This model was used to assess the interplay between temperature and parasitic current effects, but the simpler phenomenological model was preferred to determine the central values.

\subsection{Field-rise model error}

The understanding of the field variations during a fill is very different for the various years. The real relevance of these effects was realized only in 1995 with the installation of the NMR probes in the tunnel and the possibility to compare the BOF and EOF energy calibrations. This is reflected in the estimation of the error on this energy correction for each dataset.

The correlation between energy points and years has been estimated by changing the model parameters and observing the resulting energy variations. When this was not possible the error estimation has been driven by the final use of the errors: the direct influence on the determination of the mass and width of the $\mathrm{Z}$ resonance has been studied and then converted back to a centre-of-mass energy error and a corresponding correlation coefficient. If the correlation coefficient could not be precisely determined a value has been adopted which shares the uncertainty between mass and width. In such cases it has been verified that the final $m_{\mathrm{Z}}$ and $\Gamma_{\mathrm{Z}}$ errors, from the systematic study in question, have less than $10 \%$ sensitivity to the choice of correlation coefficients. It should be noted that whenever a monotonic variation over the full length of a fill is converted into an error on the average energy, a reduction of roughly a factor of two has to be applied on the change induced by the variation to reflect the luminosity-weighting.

The most important tool to estimate the error on the field rise model is the set of BOF-fills. The comparison of field drift predicted by the model with the direct measurement is used to set a limit on the error from any unknown effects.

In addition the model uncertainties have been evaluated by varying the parameters of the phenomenological model (temperature coefficients and binning of distribution) within their errors. Checks have also been performed using the deterministic model and the direct distribution of the NMR48 readings.

\subsubsection{Rise scatter and model variations}

The comparison of the phenomenological model prediction with the 6 BOF-fills (Fig. 13) yields an average offset [rise(model predicted) - rise(measured)] of $0.0 \mathrm{MeV}$ with a scatter of $0.4 \mathrm{MeV}$ (beamenergy). Studies performed by selecting at random sets of six fills in 1995 and using the NMR48 data as an estimate of the 'true' rise, show that the expected average scatter of the offset is $1.2 \mathrm{MeV}$, suggesting that the actual scatter between model and measurement for the six BOF-fills was fortuitously small. A value larger than $0.4 \mathrm{MeV}$, around or exceeding $1 \mathrm{MeV}$, is indicated also by the comparison with other 
models such as the deterministic model or the use of the NMR48 data directly. The value of $1.2 \mathrm{MeV}$ (beam-energy) has been chosen for the 1995 dataset as representative of the rms of the offset between the modelled and measured rise. Taking account of the sample size and the necessary conversion to go to the luminosity-weighted centre-of-mass energy, this translates into an error of $0.5 \mathrm{MeV}$ on the absolute centre-of-mass energy scale.

For the 1993 energy scan and the 1994 peak runs the amount of information available is reduced and is has been assumed that the error is equal to the rise predicted by the model, that is $2.6 \mathrm{MeV}$ for 1993, correlated between energy points, and $3.3 \mathrm{MeV}$ for 1994. The inter-year correlations are taken to be at the 50\% level. For 1993 and 1994 a few fills were available where, during Machine Development (MD) studies, RD measurements were performed over several hours (see Table 2). These sets of measurements are not representative of the typical physics fill as in most cases the first RD happened at the end of a physics fill and the last one several hours later, that is in a regime where only small field drifts are expected. Nevertheless, with these reservations, the comparison of the model with these fills supports the above error assignments.

To estimate the uncorrelated part of the error, i.e. that which mostly affects the $\mathrm{Z}$ width, the systematic differences between the $\mathrm{P}-2$ and the $\mathrm{P}+2$ sample have been studied.

For 1995 three different checks have been performed:

- Determining the change in the average energy at each off-peak point when using alternative models such as the deterministic model or functional fits to the RD data.

- Tuning the phenomenological model using only the $\mathrm{P}-2(\mathrm{P}+2)$ fills and applying it to the $\mathrm{P}+2(\mathrm{P}-2)$ fills to check the changes in average energy.

- Using the NMR48 data directly and checking the change in the average energy with respect to the phenomenological model.

From the observed energy dependence of the changes an error contribution equivalent to $0.4 \mathrm{MeV}$ on $\Delta \Gamma_{\mathrm{Z}}$ was assigned.

For 1993 the variations with different models were used as an estimate of the uncorrelated energy errors: the various models give differences ranging from 0.9 to $1.2 \mathrm{MeV}$. In particular the error due to the approximate assumption that temperature and parasitic-current contributions to the field drift are independent is monitored by the comparison with the deterministic model (see Table 3). From these sources, a contribution equivalent to an error on the width of $1 \mathrm{MeV}$ was taken. The larger error in 1993 can be partially accounted for by the observation that the average length of a $\mathrm{P}+2$ fill was significantly longer than the average length of the fills at $\mathrm{P}-2$ and that the $\mathrm{P}+2$ calibrations occurred correspondingly later in the fill.

\begin{tabular}{|c|c|c|}
\hline \multirow{2}{*}{ Year } & \multicolumn{2}{|c|}{$\Delta E_{\mathrm{CM}}(\mathrm{MeV})$} \\
\cline { 2 - 3 } & $\mathrm{P}-2$ & $\mathrm{P}+2$ \\
\hline 1993 & 0.0 & -1.8 \\
1995 & -0.4 & -0.2 \\
\hline
\end{tabular}

Table 3: The difference of the average fill centre-of-mass energy computed with the phenomenological model and with the deterministic model for the off-peak datasets

The model depends on the number of bins and bin-size for time of day and time into fill: these have been varied between 1 and 10 bins and the largest deviation was $0.3 \mathrm{MeV}$ for the peak energy and less than $0.1 \mathrm{MeV}$ for the off-peak points.

\subsubsection{Uncertainties in temperature coefficients}

The temperature coefficients of the NMR48 pseudo-device affect the model as they are used to subtract the part of the measured field variation which is due to temperature effects at a given time-of-day and time-into-fill, and thereby determine the residual effect due to the parasitic currents. The associated variations are studied by changing $C_{\mathrm{eff}}^{48}$ and $C_{\mathrm{T}}^{48}$ within the limits described in Section 5.4. The changes due to the variation of the two coefficients are added in quadrature and shown in Table 4. The larger shift in 1995 is partially understood as an effect related to a greater sensitivity to the temperature coefficients 
for the fills with an initial bending modulation. The level of correlation is taken to be $75 \%$ between energy points and between years. The uncertainty on the energy rise deriving from the imprecision in the modelling of the ring temperature coefficients is accounted for separately. The energy changes due to variations of the ring coefficients $C_{\mathrm{eff}}^{\text {ring }}$ and $C_{\mathrm{T}}^{\text {ring }}$, within the limits described in Section 5.4, are added in quadrature. To be conservative, the uncertainties of the ring and NMR48 temperature coefficients have been assumed to be uncorrelated. It is worth noting that the error is dominated by the uncertainty on $C_{\text {eff }}$ and not by the error on $C_{\mathrm{T}}$. These variations, shown in Table 5 , are taken as the size of the error with $75 \%$ correlations between years and energy points.

\begin{tabular}{|c|c|c|c|}
\hline \multirow{2}{*}{ Year } & \multicolumn{3}{|c|}{$\Delta E_{\mathrm{CM}}(\mathrm{MeV})$} \\
\cline { 2 - 4 } & $\mathrm{P}-2$ & $\mathrm{P}$ & $\mathrm{P}+2$ \\
\hline 1993 & 0.6 & 0.3 & 0.6 \\
1994 & & 0.5 & \\
1995 & 1.0 & 1.0 & 1.1 \\
\hline
\end{tabular}

Table 4: The variations in centre-of-mass energy for the three years for extreme variations of the temperature coefficients of the NMR48 pseudo-device: $C_{\mathrm{eff}}^{48}$ varied by $\pm 60 \%$ and $C_{\mathrm{T}}^{48}$ varied by $\pm 50 \%$. The shifts from $C_{\mathrm{eff}}^{48}$ and $C_{\mathrm{T}}^{48}$ are then added in quadrature with appropriate luminosity weighting.

\begin{tabular}{|c|c|c|c|}
\hline \multirow{2}{*}{ Year } & \multicolumn{3}{|c|}{$\Delta E_{\mathrm{CM}}(\mathrm{MeV})$} \\
\cline { 2 - 4 } & $\mathrm{P}-2$ & $\mathrm{P}$ & $\mathrm{P}+2$ \\
\hline 1993 & 0.1 & 0.4 & 0.4 \\
1994 & & 0.2 & \\
1995 & 0.4 & 0.3 & 0.4 \\
\hline
\end{tabular}

Table 5: The variations in centre-of-mass energy for the three years for extreme variations of the temperature coefficients of the ring: $C_{\mathrm{eff}}^{\text {ring }}$ varied by $\pm 30 \%$ and $C_{\mathrm{T}}^{\text {ring }}$ varied by $\pm 50 \%$. The shifts from $C_{\mathrm{eff}}^{\text {ring }}$ and $C_{\mathrm{T}}^{\text {ring }}$ are then added in quadrature with appropriate luminosity weighting

\subsubsection{Bending modulations}

An error component relevant only for the 1995 datasets is the uncertainty on the modelling of the bending modulation. The effect of varying the jump induced by a bending modulation by $\pm 1.0 \mathrm{MeV}$ around the central value of $2.5 \mathrm{MeV}$ induces a change of $0.0,1.4,0.3 \mathrm{MeV}$ (centre-of-mass energy) for $\mathrm{P}-2, \mathrm{P}, \mathrm{P}+2$ respectively. The whole variation is taken as the error with $75 \%$ correlation between energy points.

\subsubsection{Summary}

In addition to studying the effect of parameter variations and alternative models on the central values of the energies themselves, the compatibility of each variant with the 1995 BOF-fills was checked. In general good agreement was observed. The largest discrepancy, $\Delta E_{\mathrm{CM}}=1.4 \mathrm{MeV}$ i.e. slightly above two standard deviations, was observed when allowing the NMR48 temperature coefficients to span their allowed range. Such discrepancies indicate that there are anticorrelations between the error sources considered. The error assignment is conservative in the sense that these anticorrelations have been ignored.

Table 6 summarises the different components of the field rise error assignment, expressed in terms of $m_{\mathrm{Z}}$ (or $E_{\mathrm{CM}}$ for 1994) and $\Gamma_{\mathrm{Z}}$. The equivalent uncertainties on each energy point and accompanying correlations are given in Table 19. The main impact of the uncertainty of the field rise is on $m_{\mathrm{Z}}$. 


\begin{tabular}{|c|c|c|c|c|c|c|}
\hline \multirow[b]{2}{*}{ Component } & \multirow[b]{2}{*}{ How assigned } & \multicolumn{2}{|c|}{1993} & 1994 & \multicolumn{2}{|c|}{1995} \\
\hline & & $\begin{array}{r}\Delta m_{\mathrm{Z}} \\
(\mathrm{N}\end{array}$ & $\begin{array}{l}\Delta \Gamma_{\mathrm{Z}} \\
V)\end{array}$ & $\begin{array}{l}\Delta E_{\mathrm{CM}} \\
(\mathrm{MeV})\end{array}$ & $\begin{array}{r}\Delta m_{\mathrm{Z}} \\
(\mathrm{M}\end{array}$ & $\begin{array}{l}\Delta \Gamma_{\mathrm{Z}} \\
\mathrm{V})\end{array}$ \\
\hline $\begin{array}{l}\mathrm{B} \text { rise scatter and model variations } \\
\text { (including } \mathrm{T} \text { factorization) }\end{array}$ & $\begin{array}{l}\text { BOF-fills } \\
\text { Model variants } \\
\text { Full rise/ MD }\end{array}$ & 2.6 & 1.0 & 3.3 & 0.5 & 0.4 \\
\hline NMR48 temperature coefficient & Variation of coeff. & 0.6 & 0.3 & 0.5 & 1.0 & 0.5 \\
\hline Ring temperature coefficient & Variation of coeff. & 0.3 & 0.2 & 0.2 & 0.4 & 0.2 \\
\hline Bending modulation jump & Variation of jump & & & & 0.2 & 0.2 \\
\hline Total & & 2.7 & 1.1 & 3.3 & 1.2 & 0.7 \\
\hline
\end{tabular}

Table 6: The contribution of the various components of the magnetic field rise error to the final error on the $\mathrm{Z}$ mass and width, and the absolute energy scale in 1994.

\section{$7 \quad$ The accelerator size}

The LEP storage ring is based on a 'strong focusing lattice' which keeps the particles oscillating on stable orbits around the ring. The particular orbit passing on average through the centre of all quadrupoles is called the central orbit. Particles moving on this orbit sense the magnetic field of only the bending dipoles and corrector coils because there is no field on the quadrupole axis. At LEP the particles are ultra-relativistic and they circulate on a stable orbit synchronous with the frequency of the accelerating $\mathrm{RF}$ fields, which is a multiple of the revolution frequency. Since the speed of the particles is constant to a very high degree, the length of their orbits is fixed by the RF frequency ${ }^{7}$. If the positions of the magnetic elements change, for example due to movements of the tunnel structure, then, given that the length of the actual orbit is fixed, the particles will move away from the centre of the quadrupoles. Hence they will sense an additional magnetic field which attempts to restore the particle trajectory to the central orbit. This extra deflection will affect the average beam-energy of the particles. Quantitatively the energy change as a function of time $\Delta E(t)$ is related to the change of 'ring circumference' $(C)$ by

$$
\frac{\Delta E(t)}{E_{0}}=-\frac{1}{\alpha} \frac{\Delta C(t)}{C_{0}}
$$

The change of circumference, relative to the length of the central orbit $\left(C_{0}\right)$, is related to the change of energy, relative to the energy defined by the dipole-field $\left(E_{0}\right)$, through the momentum compaction factor ${ }^{8)} \alpha=(1.86 \pm 0.02) \cdot 10^{-4}$ [17]. Therefore, small changes of the LEP circumference are amplified by four orders of magnitude into measurable changes of the average beam-energy.

\subsection{Gravitational deformation of LEP}

The concrete LEP tunnel is distorted by the effects of local deformations of the surrounding geological formations. In particular, tidal effects, due to the combined gravitational attraction of the Sun and the Moon, which can cause, at Geneva latitudes, distortions of up to $10^{-8}$ of the local Earth radius, change the size of LEP in an appreciable way. The change in beam-energy due to gravitational effects can be parametrized as

$$
\frac{\Delta E}{E}=k_{\mathrm{G}} \Delta g_{\mathrm{m}}
$$

\footnotetext{
7) At LEP1 the RF frequency was kept constant during physics fills.

8) The momentum compaction factor depends on the optics used, which was the same for 1993, 1994 and 1995 . The relatively large amplification is mostly due to the strong focusing of the LEP optics.
} 

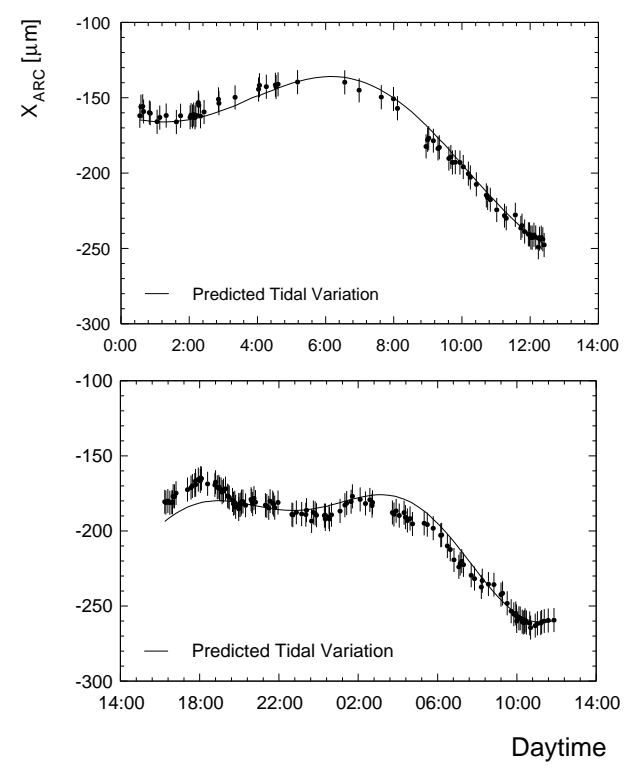

Figure 14: Evolution of the horizontal orbit position for two LEP fills. The predicted tidal variations are indicated by the solid lines. In the top figure (fill 1694) the agreement is excellent. In the bottom figure (fill 2260) the tidal effects cannot explain all the orbit movements. The errors on each point are estimates.

where $\Delta g_{\mathrm{m}}{ }^{9)}$ is the correction to be applied to the measured local acceleration due to gravity [11] and $k_{\mathrm{G}}=(-82 \pm 4) /\left(\mathrm{ms}^{-2}\right)$ is a coefficient estimated from the measured deformation of the LEP ring as detected by the Beam Orbit Measurement system [18]. The reliability of the modelling of such deformation has been proven in dedicated experiments, by comparison with energy changes as measured by the RD technique [12].

To evaluate the effect of a possible phase-shift of the model (due for example to the finite binning in time) the changes in the energy due to a relative change of \pm 7.5 minutes, corresponding to half of the recording interval, have been modelled: the largest change on the average centre-of-mass energy is $0.2 \mathrm{MeV}$. The effect of the uncertainty on $k_{\mathrm{G}}$ is determined by changing the parameter by one standard deviation and re-evaluating the average energies. The observed difference is assigned as an error (see Table 19 for the results for every energy point).

\subsubsection{Additional checks on the BOF-fills}

Using fills with multiple RD measurements, a fit similar to the one detailed in Section 5.4.1 was used to cross-check the value of the amplitude of the tide parameter $k_{\mathrm{G}}$. With $k_{\mathrm{G}}$ left as a free parameter in the fit, a value of $(0.96 \pm 0.03) \cdot k_{\mathrm{G}}{ }^{\text {std }}$ was obtained, where $k_{\mathrm{G}}{ }^{\text {std }}$ is the value used in the model; again confirming the value determined in Section 7.1 .

\subsection{Hydrogeological deformation of LEP}

In 1993 it was realized that, on top of the well-established tidal effects, some additional longterm phenomena were taking place. Over periods of weeks, changes in the average LEP radius of up to several hundred microns could be detected after correcting for tidal effects. This observation was made possible by the improved reliability of the BOM electronics. While this precise monitoring of the relative orbit deviations cross-checked the expected deformations due to tidal effects, it also made it clear that there were additional contributions (see Fig. 14). In 1994, it was realized that such changes could be qualitatively correlated with the pressure of the watertable under the nearby Jura mountains and with the level of water in the lake of Geneva.

The model of the average LEP beam-energy factorizes into a correction for tidal effects and an overall correction for each fill which accounts for the measured remaining changes of the average LEP

\footnotetext{
9) At high tide $\Delta g_{\mathrm{m}}$ reaches $\pm 1.2 \cdot 10^{-6} \mathrm{~ms}^{-2}$.
} 
radius. The correction is

$$
\Delta E=K_{\text {radius }} \Delta R
$$

where $\Delta R$ is the measured variation of the radial beam position at the BOMs. It is proportional to the variation of the radius of LEP. The coefficient $K_{\text {radius }}$ follows from the definition of the horizontal dispersion and is given by $K_{\text {radius }}=E / D_{\mathrm{x}}=(0.077 \pm 0.002) \mathrm{MeV} / \mu \mathrm{m}$ (at $\left.45 \mathrm{GeV}\right)$, where $D_{\mathrm{x}}$ is the horizontal dispersion at the BOMs. Its accuracy is limited to $2 \%$ by systematic effects.

The fill-to-fill error on the LEP radius change $\Delta R$ has been estimated to be $15 \mu \mathrm{m}$, which contributes a negligible error to the average energy considering that several hundred fills are used in the analysis.

\section{Additional contributions to the dipole fields}

Besides the dominant effect of the dipoles and quadrupoles, two effects have been found to influence the total bending field around the LEP ring: the field of the horizontal corrector coils used to perform fine steering of the particle orbits, and the stray field of the power-bars distributing the current to the quadrupoles of the machine. These effects are significant and need to be taken into account. The influence of the corrector coils, in particular, can be very important as often the corrector configuration used during $\mathrm{RD}$ calibration is different from the one used in physics conditions.

\subsection{Horizontal-corrector effects}

Around LEP there are several hundred small dipole corrector magnets used to modify the beam orbit in both planes. Horizontal correctors can affect the beam-energy either by contributing directly to the field integral or by modifying the orbit. In this respect, simulations show that the horizontal corrector magnets can affect the beam-energy significantly through a change in path length if the fields add up coherently. This change is proportional to the product of the corrector deflection angle and the horizontal dispersion at its location. Since the orbit length is fixed by the RF frequency, the change in path induced by a corrector forces the beam to change its radial position in the quadrupoles. The additional fields seen by the beam in the quadrupoles lead to a change in energy.

The LEP vertical and horizontal betatron tunes are slightly different in physics and in calibration modes, requiring in general different corrector settings. Modelling of the effect of correctors on the beamenergy is therefore necessary.

A controlled experiment during an RD measurement proved that the modelling of the effect on the beam-energy of changing the corrector settings is reasonably accurate [19] as can be seen in figure 15 . The effects of varying corrector strength and distribution are included in the model as relative changes within a fill while the systematic tracking of the absolute value of the beam energy is done by using the average orbit deformations described in Section 7.2.

Since the effect of correctors remains rather uncertain, the systematic error was calculated by assuming that horizontal correctors affect the energy either by their field contribution or by their influence on the orbit length. The results shown in Table 7 are taken as the error. The values are uncorrelated between years and $75 \%$ correlated between energy points where the sign of the correlation is taken from the relative signs of the shifts of Table 7 .

\subsection{QFQD compensation-loop effects}

The QFQD compensation loop (see Section 2.7) was used in 1993 and in 1994 (although it was not always on), and was turned off in 1995. For 1995 the effect of the stray field is constant and therefore absorbed in the overall normalization. For 1993 and 1994 the beam energy is corrected for the current, $I_{\mathrm{QFQD}}$, flowing in the QFQD compensation loop using

$$
\Delta E_{\mathrm{b}}=k_{\mathrm{QFQD}} \cdot \Delta I_{\mathrm{QFQD}} \text {. }
$$

The coefficient $k_{\mathrm{QFQD}}$, determined in dedicated experiments [3], has been revised in the light of the improved modelling of the LEP energy and it agrees with the theoretical prediction. The value used here is $0.04 \pm 0.01 \mathrm{MeV} / \mathrm{A}$ where the error is determined from the spread of the experimental results. 

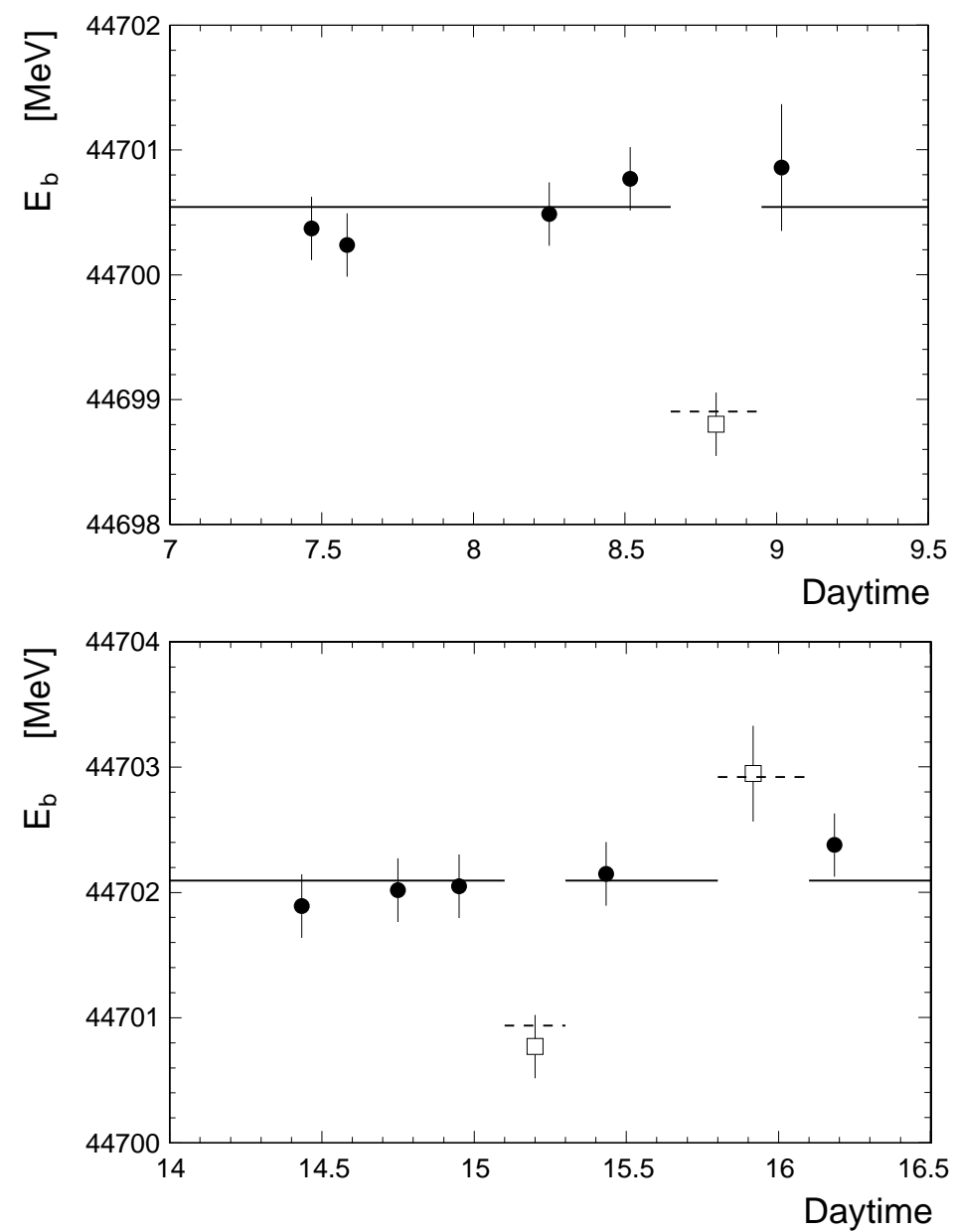

Figure 15: Evolution of the beam-energy $E_{\mathrm{b}}$ as a function of time in LEP fills 3702 (top) and 3719 (bottom). $E_{\mathrm{b}}$ is corrected for tides and magnet temperature. All RD measurements plotted as circles correspond to the reference orbit with a fixed corrector pattern. The solid lines indicate the average energy on the reference orbit. RD measurements performed with different corrector settings are indicated by squares. The dashed lines represent the energy shifts predicted from the orbit lengthening due to the change of corrector patterns with respect to the reference orbit. 


\begin{tabular}{|c|c|c|c|}
\hline \multirow{2}{*}{ Year } & \multicolumn{3}{|c|}{$\Delta E_{\mathrm{CM}}(\mathrm{MeV})$} \\
\cline { 2 - 4 } & $\mathrm{P}-2$ & $\mathrm{P}$ & $\mathrm{P}+2$ \\
\hline 1993 & +0.0 & +0.4 & -0.4 \\
1994 & & +0.2 & \\
1995 & -0.2 & -0.5 & -0.2 \\
\hline
\end{tabular}

Table 7: Average difference of centre-of-mass energy values assuming that the horizontal-corrector settings affect the beam-energy either via the orbit lengthening or via the beam deflection. The values are luminosity weighted and for all the physics fills.

\section{$9 \quad$ Uncalibrated fills}

A number of fills ended by loss of beam before calibration could be performed. For these uncalibrated fills, the absolute energy is fixed to the average energy obtained from the calibrated ones. This leads to an additional uncertainty, arising from two types of fluctuations.

First the statistical error on the mean energy estimated from the finite number, $N_{\text {cal }}$, is equal to $\sigma_{\text {cal }} / \sqrt{N_{\text {cal }}}$, where $\sigma_{\text {cal }}$ is the rms scatter of the differences $\left(E_{\text {model }}-E_{\text {polarization }}\right)$. Here $E_{\text {polarization }}$ is the end-of-fill calibration measurement and $E_{\text {model }}$ is computed from the model of Eq. (5), with a constant normalization factor $E_{\text {norm }}$ equal to the nominal value defined in Section 6.1.2. The distributions of these differences for the calibrated fills in 1993, 1994 and 1995 are shown in Fig. 16.

Secondly, the mean energy of the uncalibrated fills $\left(N_{\text {uncal }}\right)$ can also fluctuate with an error of $\sigma_{\text {cal }} / \sqrt{N_{\text {uncal }}}$. When combined to form the resulting error on the average energy of all fills, this gives the normalization error

$$
\Delta E_{\mathrm{CM}}^{\text {norm }}=\frac{N_{\text {uncal }}}{N_{\text {total }}}\left(\frac{\sigma_{\text {cal }}}{\sqrt{N_{\text {cal }}}} \oplus \frac{\sigma_{\text {cal }}}{\sqrt{N_{\text {uncal }}}}\right)=\frac{\sigma_{\text {cal }} \sqrt{N_{\text {uncal }}}}{\sqrt{N_{\text {total }}} \sqrt{N_{\text {cal }}}} .
$$

where $N_{\text {total }}=N_{\text {uncal }}+N_{\text {cal }}$ is the total number of fills. The equations above are for illustration purposes only as they neglect the effect of luminosity weighting, which is taken into account in practice. The normalization errors including the effects of luminosity weighting, are shown in Table 8.

\begin{tabular}{|c|c|c|c|}
\hline & \multicolumn{3}{|c|}{$\Delta E_{\mathrm{CM}}^{\text {norm }}(\mathrm{MeV})$} \\
\hline Year & $\mathrm{P}-2$ & $\mathrm{P}$ & $\mathrm{P}+2$ \\
\hline 1993 & 1.7 & 5.9 & 0.9 \\
1994 & - & 1.1 & - \\
1995 & 0.8 & 5.0 & 0.5 \\
\hline
\end{tabular}

Table 8: The normalization error for each centre-of-mass energy and each year. For the peak points of 1993 and 1995 the rms of all the $\mathrm{P}-2$ and $\mathrm{P}+2$ fills has been used.

\section{Centre-of-mass energy corrections}

The centre-of-mass energy at each IP is not simply twice the average beam energy for two main reasons. The first arises from the asymmetry in the distribution of the RF power which compensates for the energy lost by the beams due to synchrotron radiation. Specifically, the centre-of-mass energy can be shifted differently at each IP depending on the relative misalignments of the RF cavities in use, the phase errors on these cavities, and the disposition of RF cavities around LEP. These considerations were 

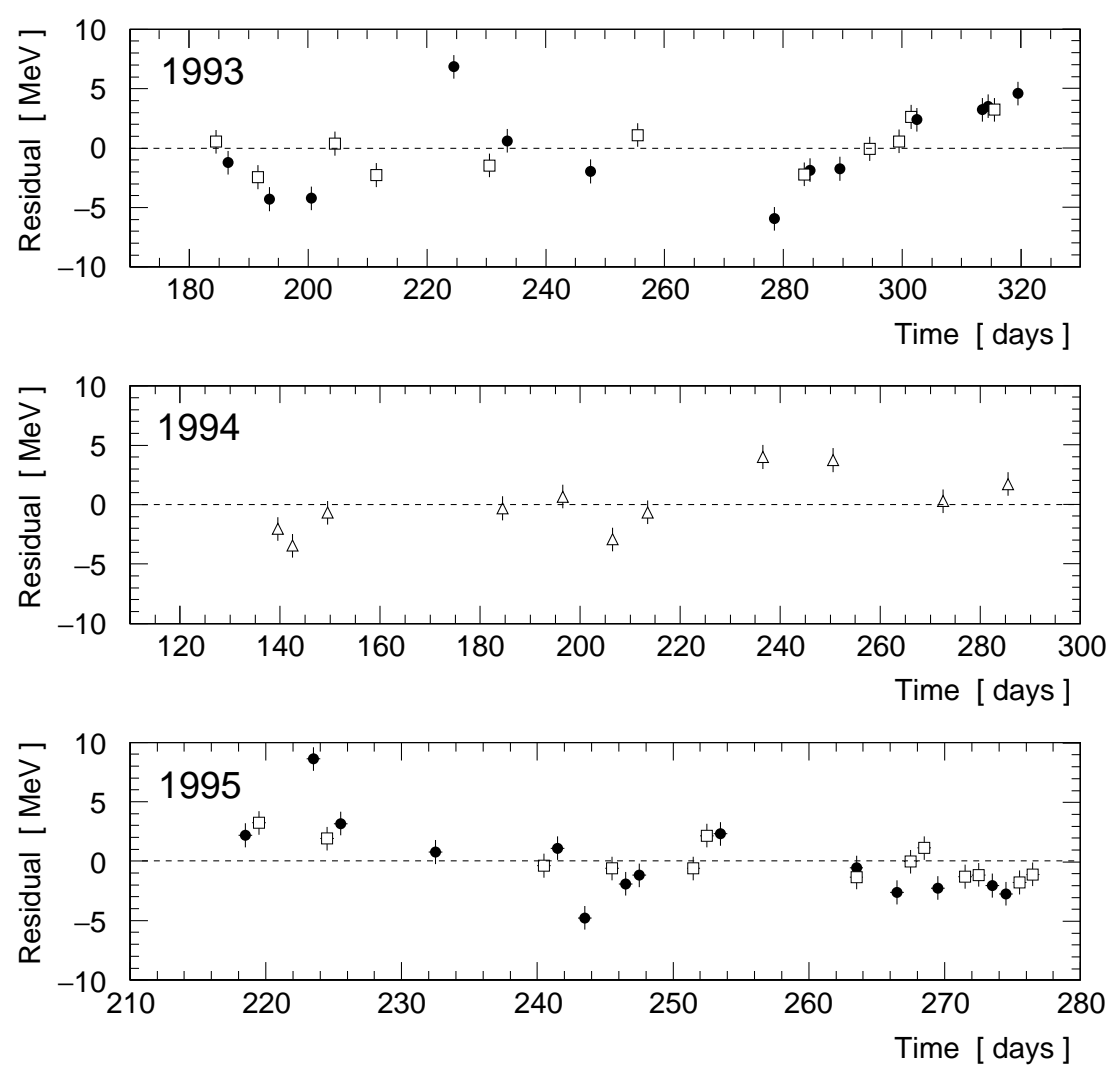

Figure 16: Differences between the beam energies measured by RD and those predicted by the model with a constant normalization factor. The error bar attached to each point is of $1 \mathrm{MeV}$. Open squares: $\mathrm{P}+2$, open triangles: $\mathrm{P}$, black circles: $\mathrm{P}-2$. 
particularly important in 1995, when the commissioning of a large number of the SC-RF cavities needed for LEP 2 operation led at times to a very asymmetric distribution of the accelerating voltage around the ring.

In 1995, the new bunch-train mode of operation introduced the second possible source of IPspecific systematic shifts in $E_{\mathrm{CM}}$. To avoid unwanted encounters between the bunches in the counterrotating bunch-trains, the beams were separated electrostatically in the vertical plane as they passed through the straight sections of LEP. As a consequence of passing off-axis through the quadrupoles near the IP, a non-negligible vertical 'dispersion' (a momentum ordering of the particles in the vertical plane) is induced. If the bunches within the train do not collide head-on, significant shifts in $E_{\mathrm{CM}}$ can occur because the sum of the energies of the colliding particles will not be equal to the average $E_{\mathrm{CM}}$. This effect was anticipated and minimized by the use of a separation scan technique to centre the beams (see Section 10.3).

As only the electron energy is routinely monitored by RD an additional correction or uncertainty common to all four IPs arises from a possible difference in energy between electrons and positrons. The measurements of this difference over the years are described in Section 10.5.

\subsection{Centre-of-mass energy corrections from the RF system}

This section presents the ingredients of the simulation used to calculate for each IP the shift in $E_{\mathrm{CM}}$ due to the interaction of the LEP beams with the RF system and other LEP components. The previously published analysis of the 1993 data [4] is summarized along with the new results from 1994 and 1995.

Until October 1995 the beam-energy loss due to synchrotron radiation (approximately $125 \mathrm{MeV}$ per turn) was compensated for by acceleration provided by RF cavities placed symmetrically at IP 2 and IP6 at diametrically opposite points on the LEP ring (see Fig. 2). Each of the groups of RF cavities was placed so that its centre lies an integral number of RF wavelengths, $\lambda_{\mathrm{RF}}$, from the IP; the RF wavelength is given by $\lambda_{\mathrm{RF}}=c / f_{\mathrm{RF}} \simeq 85 \mathrm{~cm}$, where $c$ is the speed of light and $f_{\mathrm{RF}}$ is the LEP RF frequency, $f_{\mathrm{RF}}=352204188.1 \mathrm{~Hz}$. However, to reduce the operating power the CU-RF system includes a supplementary storage cavity driven by an independent klystron operating at a slightly different frequency, $f_{2}=352294152.2 \mathrm{~Hz}$, such that the RF power oscillates between the storage and the accelerating cavity with a frequency $\left(f_{2}-f_{\mathrm{RF}}\right) / 2$. This configuration results in the CU RF system providing an accelerating voltage with a frequency $\left(f_{2}+f_{\mathrm{RF}}\right) / 2$, rather than $f_{\mathrm{RF}}$. Since the cavities were placed an integral number of $\lambda_{\mathrm{RF}}$ from each IP, they are longitudinally misaligned with respect to their operating frequency. The misalignment $\Delta \ell$ is given by

$$
\Delta \ell=c \cdot d_{\mathrm{IP}}\left[\frac{1}{f_{\mathrm{RF}}}-\frac{2}{f_{\mathrm{RF}}+f_{2}}\right] \approx 2.1 \mathrm{~cm}
$$

where $d_{\mathrm{IP}} \approx 196 \mathrm{~m}$ is the effective distance from the IP to the centre of the CU cavities. Since $\lambda_{\mathrm{RF}}>$ $2 c /\left(f_{\mathrm{RF}}+f_{2}\right)$, the cavities are too far away from the IP. The effect on the beam energies is that the incoming beam on each side of the IP will gain too much energy from the RF (about $10 \mathrm{MeV}$ extra) such that $E_{\mathrm{CM}}$ will be approximately $20 \mathrm{MeV}$ higher than the average LEP centre-of-mass energy. This effect is essentially exactly compensated for as the beams leave the IP, so that there is on average no energy offset outside IP 2 and IP6. A schematic representation of this effect is shown in Fig. 17. This cancellation is not necessarily exact, however: other geometrical effects, such as a non-zero path difference in the lengths of the LEP half-arcs IP2-IP4-IP6 and IP2-IP8-IP6 (defined as $\Delta \ell_{26}$ ), or an asymmetric distribution of RF power around LEP, can result in non-zero corrections at IP4 and IP8 as well.

To calculate the corrections to $E_{\mathrm{CM}}$ at each IP, a detailed simulation of the energy losses and gains of the beams as they travel around LEP is necessary. The modelling of the corrections is carried out by the iterative calculation of the stable RF phase angle $\phi_{\mathrm{s}}{ }^{10)}$ which proceeds by setting the energy gains of the beams in the RF cavities equal to all of the known energy losses. These losses include synchrotron radiation in the bending magnets, radiation in the wiggler magnets used to control beam emittances, and beam-energy loss in unused RF cavities. In addition, beam-loading effects, changes in the RF voltage distribution, phase errors and cavity misalignments are included.

The $1995 \mathrm{RF}$ model includes several updates specific to the new running conditions experienced during the 1995 scan:

10) The energy gain of the beams is given by $V_{\mathrm{RF}} \cdot \sin \phi_{\mathrm{s}}$, where $V_{\mathrm{RF}}$ is the total RF accelerating voltage. 


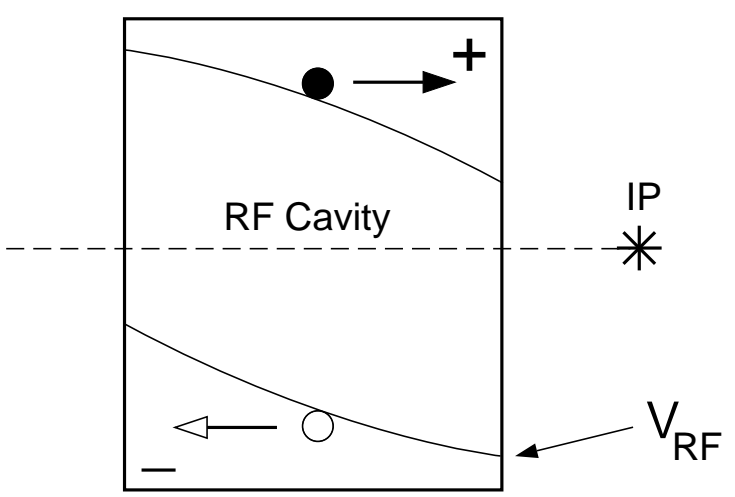

Correct alignment

$\mathrm{E}_{\text {gain }}^{+}=\mathrm{E}_{\text {gain }}^{-}$

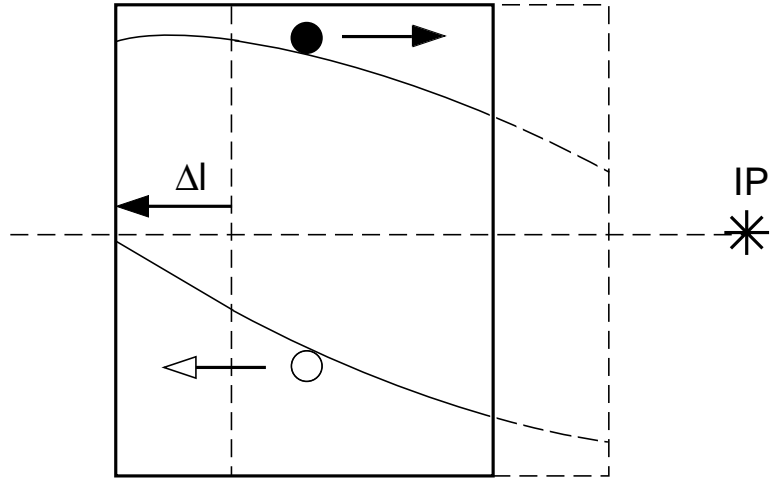

misalignment

$\mathrm{E}_{\text {gain }}^{+}>\mathrm{E}_{\text {gain }}^{-}$

Figure 17: The effects of misalignment of the RF cavities on the LEP beam-energy. In this drawing, the amplitude of the accelerating voltage $\left(V_{\mathrm{RF}}\right)$ is represented by the curved line on which the particle bunches are sitting. The top half of both figures shows the acceleration experienced by the incoming bunch, here shown as the $\mathrm{e}^{+}$bunch, whereas the bottom half of the figures shows the acceleration of the outgoing $\left(\mathrm{e}^{-}\right)$bunch at the same cavity but at a later time, after the bunches have crossed at the IP. The left figure shows the ideal situation, where the cavity sits at an integer number of RF wavelengths from the IP. Here, both bunches are given the same acceleration by the cavity. The right figure shows the situation if the cavity is moved a distance $\Delta \ell$ away from the IP but the timing of the RF accelerating voltage is kept constant. In this case, the incoming bunch arrives earlier than is optimal, and thus sees a larger accelerating field and gains a larger amount of energy $E_{\text {gain. }}$ The outgoing bunch $\mathrm{e}^{-}$arrives later than is optimal, and thus sees a smaller accelerating field and has a smaller $E_{\text {gain. }}$. If the misalignment were the same on both sides of the IP (as it is for the CU cavities in LEP), $E_{\mathrm{CM}}$ at this IP would be higher than expected from this effect.

- The introduction of the bunch-train running has necessitated the independent simulation of $\phi_{\mathrm{s}}$ for each bunch in the bunch trains, which takes into account the details of interbunch spacing and the different bunch positions on the RF wave.

- The first series of high-gradient, SC cavities, which are needed for LEP2 running, has been added, including all of their alignment, phase, and voltage calibration information. These cavities do not suffer from the longitudinal misalignment problems of the CU-RF as they operate with a single frequency.

- The use of a small number of high-gradient cavities to provide the necessary acceleration resulted in many of the CU cavities being unused during 1995. Thus, a means of describing the interaction of the beams with the unused cavities had to be added to the model. The best description was achieved if the bunches deposit energy in the unused cavities equivalent to the measured peak induced voltage, but do not, on average, interact with the stored energy.

- A new diagnostic tool, $R_{B O M}$, combines the measured size of the electron-positron difference orbit $\Delta^{+-}$on both sides of IP2 and IP6 into a ratio which is sensitive to the phasing of both individual RF units and the relative phase of the RF between IP2 and IP6. The orbit difference is directly proportional to the difference in energy of the two beams. An example of the energy difference as the beams travel around LEP is shown in Fig. 18. The definition is:

$$
R_{\mathrm{BOM}}=\frac{\left|\Delta_{\text {IP2 Left }}^{+-}\right|+\left|\Delta_{\text {IP2 Right }}^{+-}\right|}{\left|\Delta_{\text {IP6 Left }}^{+-}\right|+\left|\Delta_{\text {IP6 Right }}^{+-}\right|} .
$$

where $\Delta^{+-}=r_{\mathrm{e}^{+}}-r_{\mathrm{e}^{-}}$with $r$ being the radial displacement at the end of the LEP curved section measured by the BOM sensors. Based on the modelled energy loss and the known horizontal dispersion at the BOM position, this quantity can also be calculated in the RF model. Comparison with the measured values places powerful constraints on potential phase errors within the RF system.

Some of the model parameters are known with only limited precision, and, in addition, varied 


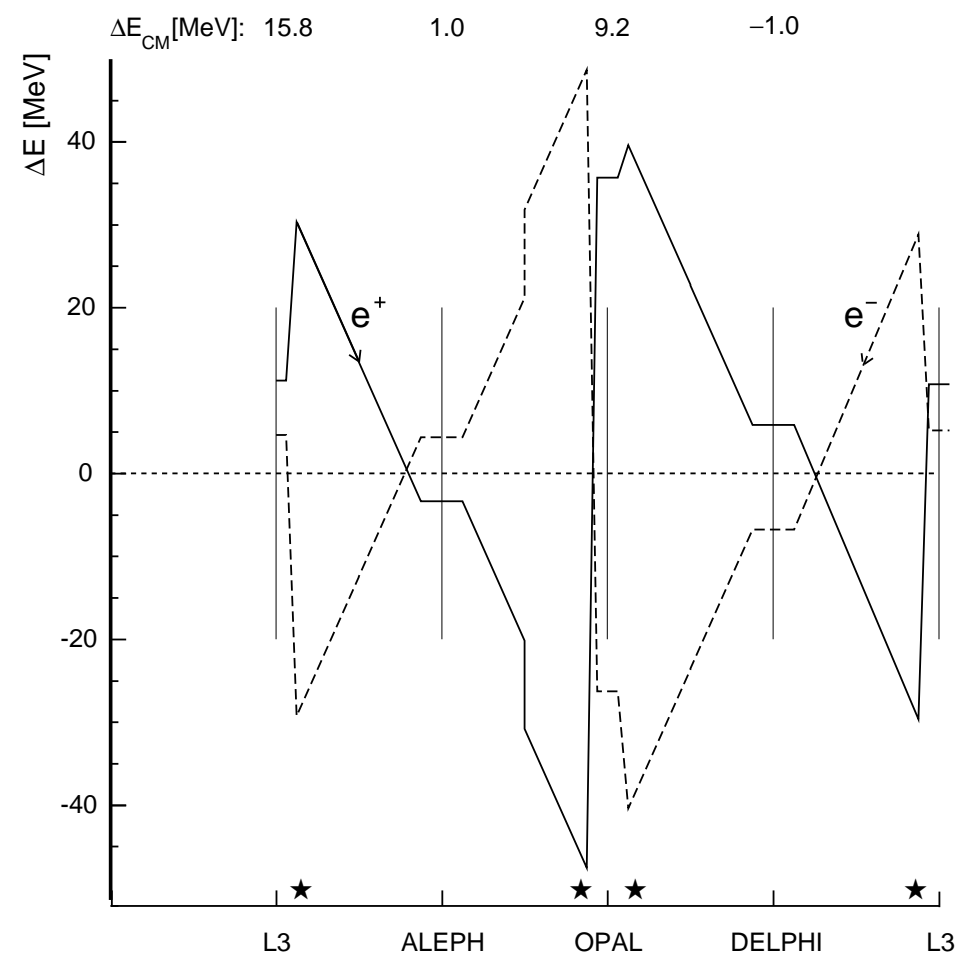

Figure 18: The evolution of the energy of each of the counter-rotating beams as they circulate in LEP, the so called energy sawtooth. The electron beam is represented by the left-going dotted line, the positron beam by the right-going solid line. The light grey lines mark the positions of the IPs. $\Delta E=0$ denotes the average energy of LEP, i.e. that given by the rest of the energy model. The orbit differences $\Delta^{+-}$used in $R_{\mathrm{BOM}}$ are measured at the points marked by a $\star$, just outside the RF sections around IP2 and IP6. The energy gains provided by the RF are clearly visible. This configuration represents a typical RF complement and centre-of-mass energy corrections for the 1995 running. 
with time. To control these, the measured value of the electron and positron synchrotron tunes ${ }^{11)} Q_{\mathrm{s}}^{ \pm}$ are compared with the value from the model, calculated from the phase angles, $\phi_{i}^{ \pm}$, in each cavity i, for each bunch, and the derivatives of the energy gain, $E_{\mathrm{i}}$, at each cavity with reference to the phase angle, $d E_{\mathrm{i}} / d \phi_{\mathrm{i}}^{ \pm}$:

$$
Q_{\mathrm{s}}^{ \pm}=\sqrt{-\frac{h_{\mathrm{f}} \alpha}{2 \pi E_{\mathrm{b}}} \sum_{i} \frac{d E_{\mathrm{i}}}{d \phi_{\mathrm{i}}^{ \pm}}} .
$$

Here, $h_{\mathrm{f}}$ is the LEP harmonic number (the ratio of the RF frequency to the revolution frequency, 31324) and $\alpha$ is the momentum compaction factor (see Section 7). Deviations between the measured and the calculated $Q_{\mathrm{s}}$ were taken as an indication of inconsistencies between input parameters, in particular the individual time-dependent unit phases.

Longitudinal shifts of the collision point in the IPs occur if the stable phase angles of the electron and positron bunches become different and vary with time. Such shifts were observed in all experiments and could be compared with the model prediction, providing another cross-check of the model's validity.

The calculations of the RF corrections use a database of patched and corrected input values where any necessary information that was not available from the variety of logging sources has been interpolated. Uncertainties arising from this can be controlled by comparison of distributions for complete and interpolated database records.

After the tuning of the RF model parameters, which required the input of time-dependent misphasing and voltage calibrations, the difference between the measured and calculated values of $Q_{\mathrm{s}}$ is finally adjusted to be zero by the application of a small voltage scaling to all of the RF voltages. Its value of $1.5 \%$ to $2.0 \%$ is consistent with the precision expected from the original voltage calibration. The rms of the difference between calculated and measured $Q_{\mathrm{s}}$ is larger in 1995 (0.0008) and 1994 (0.0006) than it was in 1993 (0.0004): this is due to the instability of the RF configuration during the 1995 scan and the poor logging quality of the 1994 running.

For the 1995 data, the mean of the distribution $R_{\mathrm{BOM}}$ (calc) $/ R_{\mathrm{BOM}}$ (meas) is 1.009 , with an rms of 0.077. Assuming a resolution of $20 \mu \mathrm{m}$ on each of the quantities $\Delta^{+-}$in Eq. (16), the width of the $\left[R_{\mathrm{BOM}}(\right.$ calc. $)-R_{\mathrm{BOM}}($ meas. $\left.)\right] / \sigma\left(R_{\mathrm{BOM}}\right)$ distribution is 2.1 . This implies that the phasing of the machine is understood to a level of approximately twice the resolution achievable from $R_{B O M}$.

The average corrections for the off-peak and peak points from 1993, 1994 and 1995 are shown in Table 9. The average bunch-specific corrections for each IP show only small bunch-to-bunch variations $(\leq 0.2 \mathrm{MeV})$, and therefore the average over the bunches in a train is used in the energy determination.

\begin{tabular}{|c|rrr|c|ccc|}
\hline \multirow{2}{*}{} & \multicolumn{6}{|c|}{ Corrections to $E_{\mathrm{CM}}(\mathrm{MeV})$} \\
\cline { 2 - 7 } & \multicolumn{3}{|c|}{1993} & 1994 & \multicolumn{3}{c|}{1995} \\
\hline LEP IP & $\mathrm{P}-2$ & $\mathrm{P}$ & $\mathrm{P}+2$ & $\mathrm{P}$ & $\mathrm{P}-2$ & $\mathrm{P}$ & $\mathrm{P}+2$ \\
\hline 2 & 19.0 & 18.8 & 19.9 & 19.1 & 14.7 & 15.0 & 14.8 \\
4 & 0.0 & -0.3 & 0.0 & -0.2 & 0.9 & 1.7 & 1.4 \\
6 & 19.5 & 19.6 & 20.1 & 18.9 & 4.4 & 4.6 & 4.6 \\
8 & 0.0 & 0.3 & 0.0 & 0.0 & -1.0 & -1.8 & -1.5 \\
\hline
\end{tabular}

Table 9: The corrections to $E_{\mathrm{CM}}$ for each LEP IP and each energy point from the RF model.

\subsection{Evaluation of errors for the RF corrections}

The constituent errors on the 1994 and 1995 analyses are discussed below. Unless explicitly mentioned, nothing is changed for 1993 from the published results [4]. The 1994 and 1995 errors are summarized in Table 10.

11) The synchrotron tune is the number of longitudinal, or energy, oscillation per turn of LEP. 
The largest systematic error on the RF corrections arises from the uncertainties on the positions of the RF cavities. The uncertainty on the alignment of the CU-cavities is taken to be $\pm 1 \mathrm{~mm}$, where the most conservative estimate of the effect is to assume that the total uncertainty on the difference in cavity positions is $2 \mathrm{~mm}$, moving the cavities coherently towards or away from the IP to calculate the effect. This led to shifts of $0.4 \mathrm{MeV}$ at IP2 and IP6 in 1994, and 0.6 MeV at IP2 and 0.2 MeV at IP6 in 1995. In 1995, alignment uncertainties of about $1.4 \mathrm{~mm}$ (obtained from repeated measurements of the cavity positions) on the superconducting cavities are also important and amount to $0.1 \mathrm{MeV}$ at IP2 and 0.5 $\mathrm{MeV}$ at IP6, leading to a total systematic error arising from RF cavity misalignment of 0.6 and $0.5 \mathrm{MeV}$ for IP2 and IP6, respectively. The corresponding error for the 1994 running is negligible because the total accelerating voltage provided by superconducting cavities was small. Since the source of this error is geometrical, it is taken to be $100 \%$ correlated between energy points and years of running.

A very important systematic uncertainty in 1995 results from the discrepancy between the observed and predicted longitudinal movements of the collision point and the difference in collision points between bunches. The measurements by the LEP experiments of the position of the average interaction point measured along the beam axis ( $z$ coordinate) show good agreement except for a small period at the end of the scan, when all CU cavities around IP6 were unused and new superconducting RF cavities were operated instead. The model also predicts smaller differences between the individual bunch collision points, about $0.5 \mathrm{~mm}$ between the first and the last bunch in a train, than are measured from the data, where differences up to $1 \mathrm{~mm}$ are observed. The average energy of the various bunches was shown to be equal to within $0.1 \mathrm{MeV}$, with an rms of $0.3 \mathrm{MeV}$, by means of RD measurements. This, however, does not exclude local effects due to the RF system. Limits on the possible shifts in collision energy arising from collective and bunch-to-bunch shifts in stable phase are obtained by using the measured vertex positions as an input to the RF model to constrain the bunches to sit at a $\phi_{\mathrm{s}}$ which identically reproduces the observed collision positions [20]. The systematic errors due to this effect are $0.8,0.6,0.1$, and 0.6 MeV for IPs 2, 4, 6, and 8, respectively. These errors include small discrepancies in the $Q_{\mathrm{s}}$ values of the individual bunches in a train. The errors are assumed to be $100 \%$ correlated between IPs and $50 \%$ correlated between energy points.

The residual rms spread of $\left(Q_{\mathrm{s}}\right.$ (calc.) $-Q_{\mathrm{s}}$ (meas.)) was larger in 1994 and 1995 by $50 \%$ and $100 \%$ respectively compared to 1993. This, and the need to re-scale the RF voltages to obtain agreement between the average measured and calculated $Q_{\mathrm{s}}$, indicates an uncertainty in the RF voltages and phases, which is taken into account by assuming the full normalization correction as the error on the voltages. This resulted in errors on centre-of-mass energy of $0.4 \mathrm{MeV}$ at IP2 for both years, 0.4 and $0.1 \mathrm{MeV}$ at IP6 in 1994 and 1995 respectively, and much smaller errors $(<0.1 \mathrm{MeV})$ at IP4 and IP8. These errors are $100 \%$ correlated between energy points and IPs, but are taken to be uncorrelated between years. Separate studies of the agreement between measured and modelled $Q_{\mathrm{s}}$ at the various energy points showed no significant differences.

Measurements made in 1996 during the high-energy running of LEP have revealed a potential discrepancy between the modelled and actual energy loss by the circulating beams. In particular, a difference of approximately 5\% was observed between the energy loss calculated from the size of the horizontal orbit excursions measured by the BOM system and that assumed from the optical modelling of LEP. To estimate the effect of a global change in the rate of energy loss on the RF corrections to $E_{\mathrm{CM}}$, the loss was changed in the simulation by $5 \%$ and the full shifts in $E_{\mathrm{CM}}$ taken as the uncertainty from this effect. This results in an additional error of $0.3 \mathrm{MeV}$ at IP2 for 1995, and $0.6 \mathrm{MeV}$ errors at IP2 and IP6 for 1993 and 1994 which are correlated between these two IPs for these years.

For 1995 , the measured quantity $R_{\mathrm{BOM}}$ provides a stringent constraint on possible phase errors between and within the RF at IP2 and IP6. To estimate the possible energy errors due to misphasing, the relative phases of the CU cavities at IP2 and IP6 were shifted in a way to move $R_{\mathrm{BOM}}$ to two standard deviations from its central value. The size of the effect is a negligible $0.1 \mathrm{MeV}$ at IP2 and $0.0 \mathrm{MeV}$ at IP6. For 1994, however, the $R_{\mathrm{BOM}}$ information is not available, and a $5^{\circ}$ shift in the relative phases between the two IPs is used to estimate the error due to misphasing. In this case, the error is $0.5 \mathrm{MeV}$ at IP2 and IP6, anticorrelated between the two. Possible errors due to misphasing are not correlated between years.

The recent measurement of cavity positions [21] also includes an estimation of the possible pathlength difference of the two half-arcs of LEP, from IP2 to IP6 via IP 4 or IP $8\left(\Delta \ell_{26}\right)$. The difference in path length was measured to be zero, with an error of $1 \mathrm{~mm}$. This result can be applied retrospectively to the 1993 analysis, such that the resulting error on the centre-of-mass energy is reduced from $1 \mathrm{MeV}$ to $0.1 \mathrm{MeV}$ for IP4 and IP8. The error is anticorrelated between the two IPs, and is $100 \%$ correlated between energy points and years. 
Additional small errors arise from missing input data, from a small difference in average $Q_{\mathrm{s}}$ of electrons and positrons, and from the errors on model input parameters. These are quantified in Table 10.

\begin{tabular}{|c|c|c|c|c|}
\hline Error & & $\Delta E_{\mathrm{CM}}(\mathrm{MeV})$ & $\mathrm{P} \pm 2$ Corr. & Comments \\
\hline RF misalignment & $\begin{array}{l}\text { IP2 } \\
\text { IP6 }\end{array}$ & $\begin{array}{l}0.6[0.4] \\
0.5[0.4]\end{array}$ & $\begin{array}{l}100 \% \\
100 \%\end{array}$ & uncorrelated \\
\hline $\begin{array}{l}\text { Bunch }\left\langle z_{0}\right\rangle \text { shifts } \\
\text { within a train }\end{array}$ & $\begin{array}{l}\text { IP } 4,8 \\
\text { IP } 2 \\
\text { IP6 }\end{array}$ & $\begin{array}{l}0.6 \\
0.8 \\
0.1\end{array}$ & $\begin{array}{l}50 \% \\
50 \% \\
50 \%\end{array}$ & all IPs correlated \\
\hline Voltage scale & $\begin{array}{l}\text { IP2 } \\
\text { IP6 }\end{array}$ & $\begin{array}{l}0.4[0.4] \\
0.1[0.4]\end{array}$ & $\begin{array}{l}100 \% \\
100 \%\end{array}$ & IP2 and IP6 corr. \\
\hline Energy loss & $\begin{array}{l}\text { IP2 } \\
\text { IP6 }\end{array}$ & $\begin{array}{l}0.3[0.6] \\
0.0[0.6]\end{array}$ & $\begin{array}{l}100 \% \\
100 \%\end{array}$ & correlated \\
\hline$\Delta \ell_{26}$ & $\mathrm{IP} 4,8$ & $0.1[0.1]$ & $100 \%$ & IP4 and IP8 anticorr. \\
\hline Missing data & & $0.1[0.2]$ & $50 \%$ & all IPs correlated \\
\hline$Q_{\mathrm{s}}\left(\mathrm{e}^{-}\right)-Q_{\mathrm{s}}\left(\mathrm{e}^{+}\right)$ & & 0.2 & $50 \%$ & IP4 and IP8 anticorr. \\
\hline RF misphasing & $\begin{array}{l}\text { IP2 } \\
\text { IP6 }\end{array}$ & $\begin{array}{l}0.1[0.5] \\
\sim 0[0.5]\end{array}$ & $50 \%$ & IP2 and IP6 anticorr. \\
\hline$\Delta \alpha= \pm 2 \cdot 10^{-6}$ & $\mathrm{IP} 2,6$ & $0.1[0.1]$ & $100 \%$ & IP2 and IP6 corr. \\
\hline
\end{tabular}

Table 10: A summary of the systematic errors for the 1994 and 1995 analyses of the RF corrections. Errors not listed for a given IP are negligible $(<0.1 \mathrm{MeV})$. The values for 1994 are given in square brackets, and are zero if not listed. The column labelled ' $\mathrm{P} \pm 2$ Corr.' gives the correlation of the errors between energy points in 1995 . The correlations between years of LEP running are given in the text.

\subsection{Centre-of-mass energy corrections from dispersion effects}

The bunch-train mode of operation used for the 1995 energy scan required the control of a new effect. The beam-orbit deflections induced by the electrostatic separators to avoid unwanted collisions, hereafter called 'bunch-train bumps', generate vertical dispersion of opposite-sign for the two beams at the IPs. If the beams collide with a vertical offset, the centre-of-mass energy is shifted from the nominal by an amount $\Delta E_{\mathrm{CM}}[22]$ :

$$
\Delta E_{\mathrm{CM}}=-\frac{1}{2} \cdot \frac{\delta y}{\sigma_{y}^{2}} \cdot \frac{\sigma_{E_{\mathrm{b}}^{2}}}{E_{\mathrm{b}}} \cdot \Delta D_{y}^{*}
$$

where $\delta y$, the collision offset, is the vertical distance between the centre of the positron bunch and the electron bunch in collision at the IP, $\sigma_{y}$ is the individual beam size, $\Delta D_{y}^{*}$ is the difference in the vertical dispersion $\left(D_{\mathrm{e}^{+}}^{*}-D_{\mathrm{e}^{-}}^{*}\right)$ and $E_{\mathrm{b}}, \sigma_{\mathrm{E}_{\mathrm{b}}}$ are the beam-energy and spread respectively.

As $\Delta D_{y}^{*}$ is a characteristic of the bunch-train bumps and $\sigma_{\mathrm{E}}$ is set by the beam-energy and optics, the only feasible control of this effect is to reduce $\delta y$. In the 1995 energy scan, LEP was operated with four trains of three bunches per beam. Under such circumstances it is not possible to collide all bunches with zero $\delta y$ since the residual beam-beam kicks generate transverse distortions of the train [23]. It is shown in [22] that optimization of the total luminosity results in a cancellation of the individual $\Delta E_{\mathrm{CM}}$ of each collision within a train, provided that the dispersion differences are the same for each bunch.

The strategy adopted for controlling collision offsets was based on luminosity optimization using vertical separator scans [24]. From these separator scans are obtained:

- The electrostatic-separator setting giving maximum luminosity, at the time of the scan, for the given IP (optimal setting), globally and for each family. 


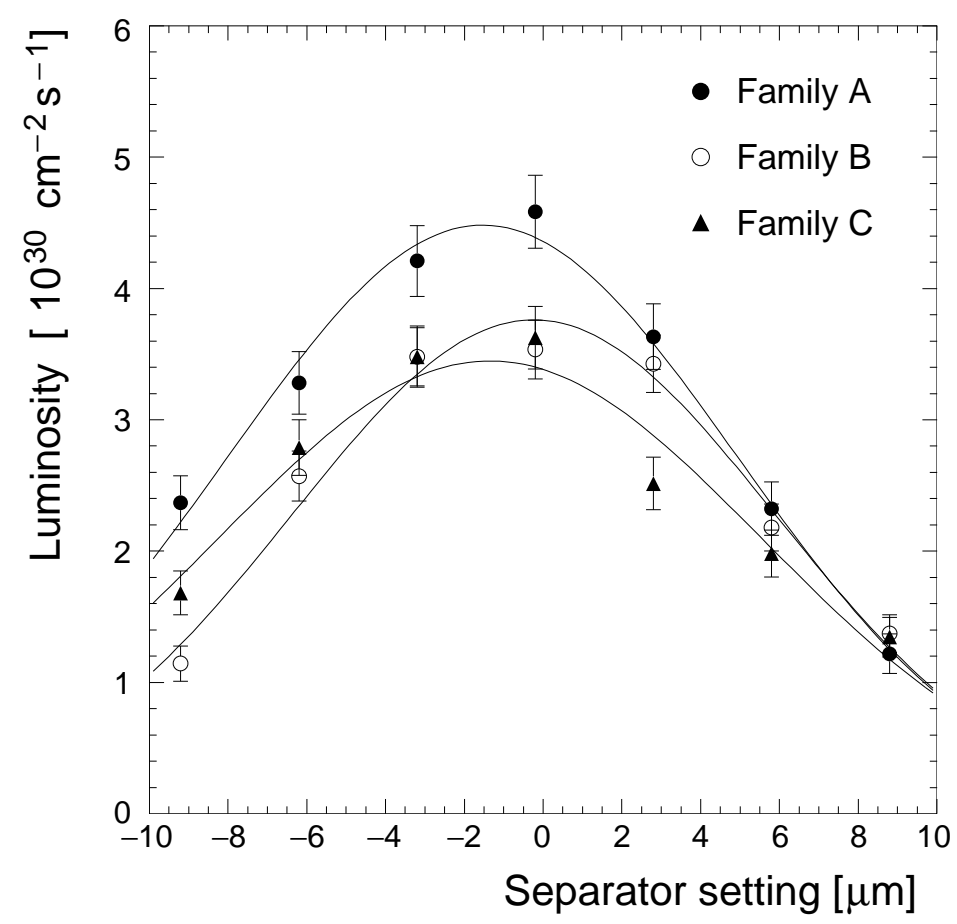

Figure 19: Typical online display of a separator scan taken at IP6. The three different sets of points correspond to the three families. The lines are Gaussian fits to the data.

- The vertical beam size, $\sigma_{y}$.

- The collision offset, $\delta y$, by comparing the setting of the separator before the scan to the optimal setting.

- The opposite-sign vertical dispersion was measured by repeating the separator scans for different RF frequencies, i.e. different beam energies.

With the knowledge of the energy spread measured as described in Section 12, these measurements are sufficient to determine the energy shifts of Eq. 18. More details of this analysis can be found in [25].

\subsubsection{Separator scans}

A separator scan consists of moving the beams vertically in opposite directions by varying the voltage of the electrostatic separators. Separator scans were performed once or twice in a fill. After a scan was completed, the separator setting was adjusted to the optimal position, that is the one maximizing the total luminosity, given by an online fit.

Fig. 19 shows a typical separator scan. The three different sets of points correspond to the three

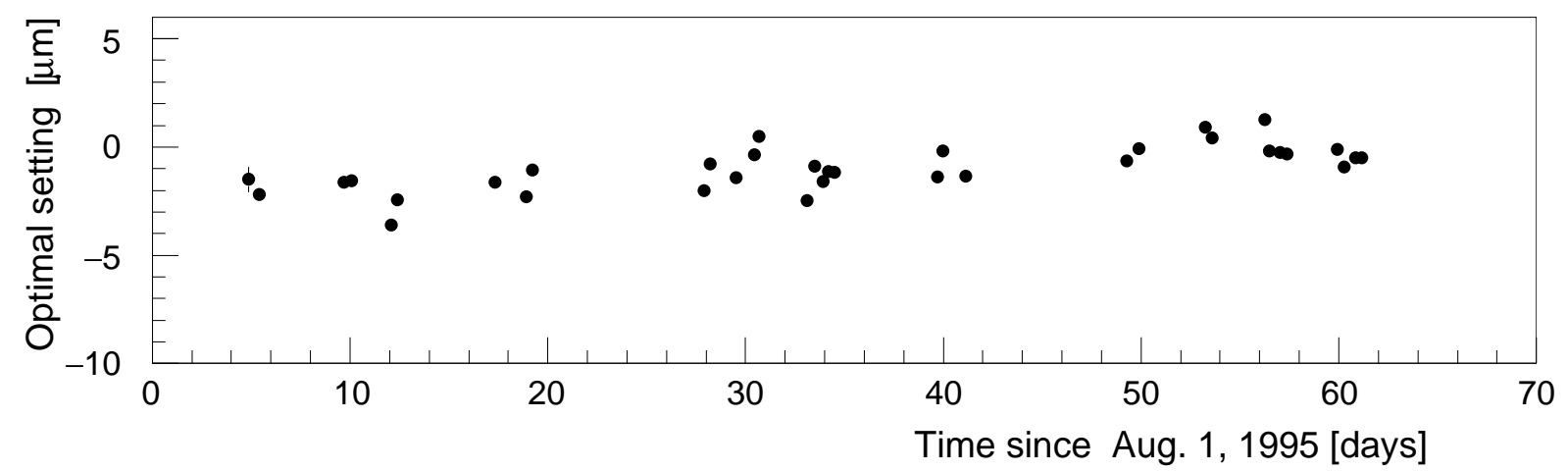

Figure 20: The stability of the optimal separator setting for IP2 during the 1995 energy scan for P-2. 
families. The shift of the maximum for family $\mathrm{B}$ with respect to families $\mathrm{A}$ and $\mathrm{C}$ is in agreement with theoretical predictions [23].

The separator-scan data were re-analysed offline to study quality, stability and other characteristics of the scans. The Gaussian fits were found to be good with a mean $\chi^{2} / D . O . F$. of $\simeq 1.2$. The typical statistical error on the optimal separator scan position per family is $0.2-0.3 \mu \mathrm{m}$. There were about 35 scans taken at each IP and at each off-peak energy point for the purpose of controlling the collision offsets.

As an example, the stability of the optimal position is shown in Fig. 20 for IP2 as a function of time into the 1995 energy-scan running period (time since 1 Aug., 1995) for P-2. The rms variation of the optimal position for each IP and each energy is given in Table 11.

\begin{tabular}{|c|c|c|c|c|}
\hline \multirow{2}{*}{} & \multicolumn{4}{|c|}{ rms separator setting $(\mu \mathrm{m})$} \\
\cline { 2 - 5 } & IP2 & IP4 & IP6 & IP8 \\
\hline $\mathrm{P}-2$ & $1.07 \pm 0.13$ & $0.86 \pm 0.11$ & $1.41 \pm 0.18$ & $0.98 \pm 0.12$ \\
$\mathrm{P}+2$ & $0.97 \pm 0.11$ & $1.11 \pm 0.13$ & $1.50 \pm 0.18$ & $1.24 \pm 0.14$ \\
\hline
\end{tabular}

Table 11: The rms variation of the optimal separator-setting position (using a Gaussian fit) for each IP.

\subsubsection{Vertical beam sizes}

Ideally the vertical beam size can be extracted directly from the width of the luminosity curve in a separator scan. The beam size itself varies during the separator scan due to increased beam-beam forces when the collisions are not perfectly head-on at the IP being scanned. This effect was corrected for using the luminosity measured at the same time in the other IPs, resulting in much improved fits to the luminosity curves. The luminosity-weighted average of the vertical beam size, $\bar{\sigma}_{y}$, determined in this way is used in Eq. 18 to define the scale of the energy shifts. Table 12 shows the average vertical beam size, $\bar{\sigma}_{y}$, for each IP and energy point in 1995. The significant differences in the vertical beam sizes at different IPs are likely due to the overall separator-setting scale, i.e. to the conversion of separator voltages to microns of deflection at the IP, which can differ by up to $15 \%$ between IPs. An overall difference in the separator-setting scale drops out of Eq. (18) since the collision offset, $\delta y$, and the dispersion difference, $\Delta D_{y}^{*}$, are also subject to the same scale differences.

\begin{tabular}{|c|c|c|c|c|}
\hline \multirow{2}{*}{} & \multicolumn{4}{|c|}{$\bar{\sigma}_{y}(\mu \mathrm{m})$} \\
\cline { 2 - 5 } & IP2 & IP4 & IP6 & IP8 \\
\hline $\mathrm{P}-2$ & $3.84 \pm 0.12$ & $5.20 \pm 0.08$ & $4.75 \pm 0.11$ & $4.08 \pm 0.13$ \\
$\mathrm{P}+2$ & $3.93 \pm 0.07$ & $5.39 \pm 0.10$ & $4.76 \pm 0.07$ & $4.10 \pm 0.06$ \\
\hline
\end{tabular}

Table 12: The mean vertical beam size $\bar{\sigma}_{y}$. The errors shown are statistical only: the overall absolute scale at each IP is uncertain by up to $15 \%$.

\subsubsection{Collision offsets}

In practice, the LEP operators set the separators for each IP to the optimal setting determined from the online fit after a separator scan at that IP. For data taken before a separator scan had been performed, the settings determined from the previous fill were used. These actual settings were logged continuously. They are close, but not identical, to the true optimal settings, the differences leading to potentially dangerous collision offsets.

In order to evaluate the collision offsets and resulting energy corrections, a model of the optimal settings as a function of time is required, and was estimated as described below. Since optimal settings are known only at the time of a separator scan, this involves interpolation in time, with some uncertainty. 
First the separator scans were refitted offline in order to take into account such effects as variation of beam size and online data truncation. Then, for fills with two separator scans available (50\% of the data), the value given by the first scan was used for the first part of the fill, a linear interpolation was used between the two scans, and the value given by the second scan was used for the last part of the fill. For fills with only one scan, its value was used throughout. For fills with no scan (less than $10 \%$ of the data), the yearly average of optimal settings for the IP and energy considered was used. Uncertainties in this procedure were evaluated by using the last available optimal setting instead of these interpolations.

The luminosity-weighted collision offsets at the four IPs are shown in Table 13. The errors are calculated from the rms variation of the optimal settings. The stability of the optimal settings with time and the large number of scans performed, about 35 scans at each energy point for each IP with an rms of about 1 micron over the 1995 running period, ensure that the resulting errors are small. The optimal settings were found to be different for the two off-peak energies. Since this was not anticipated, the separator settings at the beginning of fills, being taken from the previous fill with a different energy, were systematically offset from the optimum during the first part of the 1995 running period. This was corrected for the later part of that period. The resulting corrections to the energies of the off-peak points lead to corrections to the $\mathrm{Z}$ width, determined from the 1995 data, of up to $0.6 \mathrm{MeV}$ for each experiment. The corrections have different signs for the different IPs and are correlated so that the effect for the LEP average $\mathrm{Z}$ width is smaller than $0.2 \mathrm{MeV}$.

\begin{tabular}{|c|c|c|c|c|}
\hline \multirow{2}{*}{} & \multicolumn{4}{|c|}{$\langle\delta y\rangle_{\text {lum }}(\mu \mathrm{m})$} \\
\cline { 2 - 5 } & IP2 & IP4 & IP6 & IP8 \\
\hline P -2 & $0.43 \pm 0.17$ & $0.53 \pm 0.19$ & $0.34 \pm 0.24$ & $0.18 \pm 0.16$ \\
P +2 & $-0.05 \pm 0.17$ & $-0.36 \pm 0.19$ & $0.15 \pm 0.30$ & $-0.16 \pm 0.24$ \\
\hline
\end{tabular}

Table 13: The luminosity-weighted collision offsets $\langle\delta y\rangle_{\text {lum }}$.

\subsubsection{Dispersion measurements}

The LEP beam-energy is changed by changing the RF frequency. Each beam moves by an amount proportional to the beam energy shift. Measurements of the optimal vertical separator settings before and after the RF frequency shift allow the determination of the difference $\Delta D_{y}^{*}$ in the dispersion of the two beams from the relation

$$
\Delta D_{y}^{*}=-\frac{\alpha \cdot f_{\mathrm{RF}}}{\Delta f_{\mathrm{RF}}} \cdot \Delta y_{\mathrm{opt}}
$$

where $\Delta y_{\mathrm{opt}}$ is the observed shift in the optimal separator setting when the RF frequency, $f_{\mathrm{RF}}$, is shifted by $\Delta f_{\mathrm{RF}}$. The momentum compaction factor (see Section 7 ) is denoted by $\alpha$.

An example of a dispersion measurement at IP2 is given in Fig. 21, where the offsets measured for each family are displayed for three beam energies. The dispersion is proportional to the offset difference between both off-energy measurements. The dispersion value retained is the average over the three families. The three separator scans done at nominal frequency before and after each off-frequency scan are used as a consistency check.

Dispersion measurements were made for each IP at each energy. The results (see Table 14) were averaged over the families and the energies. The fluctuations of the different measurements are much larger than their statistical error and are quite different from one IP to another. The error quoted in Table 14 is the sum of all the systematic effects investigated in reference [25]. The theoretical expectations coming from the accelerator simulation code MAD [26] are also given. The agreement between these predictions and the measurements ensures that the effect is well under control.

\subsubsection{Energy shift}

Using the results from the previous sections for beam size and dispersion difference, the sensitivity to collision offsets at each IP is determined from Eq. 18 to be $-2.3,1.3,-1.4$ and $1.6 \mathrm{MeV} / \mu \mathrm{m}$ for IP2, 4, 6 and 8 respectively. 

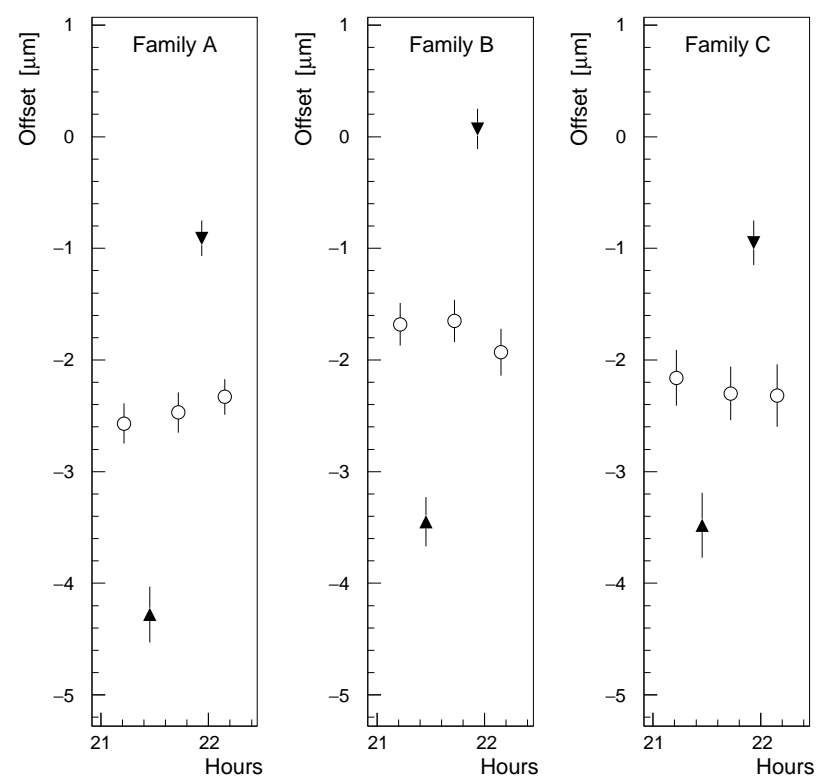

Figure 21: Example of a dispersion measurement at IP2 for the three families. The offsets for the three energies are displayed ( $\circ$ for the nominal energy, $\boldsymbol{\Delta}$ and $\boldsymbol{\nabla}$ for $\Delta f_{\mathrm{RF}}=+50$ and $-50 \mathrm{~Hz}$ respectively).

\begin{tabular}{|l|c|c|c|c|}
\hline \multirow{2}{*}{} & \multicolumn{4}{|c|}{$\Delta D_{y}^{*}(\mathrm{~mm})$} \\
\cline { 2 - 5 } & IP2 & IP4 & IP6 & IP8 \\
\hline Measurement & $2.0 \pm 0.4$ & $-2.0 \pm 0.7$ & $1.8 \pm 0.8$ & $-1.5 \pm 0.7$ \\
Theoretical prediction & 1.8 & -2.8 & 1.9 & -1.9 \\
\hline
\end{tabular}

Table 14: Dispersion difference $\Delta D_{y}^{*}$ at each IP.

This sensitivity can be combined with the collision offsets given in Table 13 to obtain an estimate of the luminosity-weighted centre-of-mass energy shifts at each IP and energy point. This is shown in Table 15. The error is due to the statistical error on the collision offset, $\delta y$, from Table 13.

\begin{tabular}{|c|c|c|c|c|}
\hline & \multicolumn{4}{|c|}{$\Delta E_{\mathrm{CM}}(\mathrm{MeV})$} \\
\hline & IP2 & IP4 & IP6 & IP8 \\
\hline $\mathrm{P}-2$ & $-0.99 \pm 0.39$ & $0.69 \pm 0.24$ & $-0.48 \pm 0.33$ & $0.29 \pm 0.25$ \\
$\mathrm{P}+2$ & $0.12 \pm 0.39$ & $-0.47 \pm 0.24$ & $-0.21 \pm 0.41$ & $-0.26 \pm 0.38$ \\
\hline
\end{tabular}

Table 15: The centre-of-mass energy correction $\Delta E_{\mathrm{CM}}$ due to dispersion effects. The error is due to the error on the determination of the collision offset $\delta y$.

\subsection{Evaluation of errors for the dispersion corrections}

In addition to the statistical errors given in Table 15, the following systematic error sources were considered. A first set of errors is uncorrelated between IPs and energies, either because they stem from luminosity statistics in the separator scans, or because they produce effects with no obvious pattern between energies and experiments:

- The errors on the measurement of $\Delta D_{y}^{*}$ 


\begin{tabular}{|l|ccc|}
\hline \multicolumn{4}{|c|}{ Errors on $\Delta E_{\mathrm{CM}}(\mathrm{MeV})$} \\
\hline & Statistical & Uncorrelated & Correlated \\
\hline IP2 & 0.39 & 0.46 & 0.31 \\
IP4 & 0.24 & 0.35 & 0.18 \\
IP6 & 0.37 & 0.34 & 0.20 \\
IP8 & 0.32 & 0.36 & 0.24 \\
\hline
\end{tabular}

Table 16: Mean global errors on $\Delta E_{\mathrm{CM}}$ for each IP. The systematic errors are labelled uncorrelated and correlated.

- The error on the measurement of the vertical beam size (except the overall scale error, which drops out). This is obtained by comparing the results obtained with and without the correction for beam size variation during the separator scans. Also, the difference in results obtained when using a time-averaged beam size for the whole running period or that measured as function of time is taken as a systematic error.

- The results from the Gaussian fit have been compared to the results of the beam size corrected fit, as well as to those of another fit (and model) which uses the LEP BEUV [27] instrument to estimate the beam size.

- Eq. 18 gives the energy shift assuming the opposite sign vertical dispersion is the same for all bunches in a train. Possible deviations from this assumption were evaluated using the experimental errors on the family-by-family dispersion measurements.

The following errors are considered fully correlated between energies and experiments:

- A possible bias resulting from the direction in which the separator voltages are scanned. This effect was studied by performing separator scans where voltages were varied in the direction opposite to the usual one.

- Variations in the method used to interpolate the optimal settings between separator scans (see Section 10.3.3). This effect is found to be similar at $\mathrm{P}-2$ and $\mathrm{P}+2$ and for each IP presumably because the drift of optimal settings during a fill is due to variations in the beam-current intensity.

The off-peak averages of these errors, as well as the statistical errors are given in Table 16 for each IP. In all cases, the systematic errors are larger than the statistical ones. The resulting errors on the energy shifts are between 0.5 and $0.7 \mathrm{MeV}$, in good agreement with design expectations [24].

\subsubsection{Dispersion effects in 1993 and 1994}

During the LEP pretzel running in 1993 and 1994, the beams were separated in the odd IPs with short vertical-separation bumps similar to the bunch-train bumps. The opposite-sign dispersion expected at the even points due to these bumps is smaller than $0.1 \mathrm{~mm}$. An unknown fraction of horizontaldispersion difference created by the pretzel separators may also couple into the vertical plane. An estimate of the opposite-sign dispersion at the collision points was obtained from dispersion measurements made with the beam position monitors. An extrapolation of the difference dispersion measured at monitors located around the collision points indicated the presence of opposite-sign vertical dispersion of between 0.2 and $0.6 \mathrm{~mm}$. The measurement accuracy is about $\pm 0.3 \mathrm{~mm}$. No precise figures are available in 1993 and 1994 for the collision offsets since the separator settings were optimized manually by the operators to tune the performance. No systematic separator scans were performed at that time. By comparing the results of empirical optimisation of the collision offsets by the accelerator operators with the separator scan results from 1995 it was concluded that any possible vertical collision offset in 1993 and 1994 would be smaller than $1 \mu \mathrm{m}$. This yields systematic errors on the centre-of-mass energy of $0.4 \mathrm{MeV}$ for 1993 and $0.7 \mathrm{MeV}$ for 1994. The correlations between the interaction points and the two running periods are unknown: $75 \%$ correlation between energy points in 1993 and 50\% correlation between years has been assumed, with no correlation between IPs. 


\subsection{Positron beam-energy}

Imperfections in the LEP lattice such as misalignments of quadrupoles, imperfections in the quadrupole and sextupole fields or asymmetries in the optical sequences combined with the energy sawtooth (see Fig. 18) can cause horizontal deviations from the ideal orbit which are different for positrons and electrons. Theoretical calculations and detailed simulations of imperfect accelerator layout lead to an upper limit of $0.3 \mathrm{MeV}$ on the maximum energy difference between the two beams (see [14], p. 119 and references therein). In 1994 a positron polarimeter was installed in LEP, while in 1993 the electron polarimeter was slightly modified in order to attempt a measurement of the positron energy which was carried out at the very end of the running period. To maximize the physics time the calibration of the positron beam-energy was performed only once or twice per year, while the RD measurements were performed routinely on the electron beam. A summary of the various measurements performed is given in Table 17. The 1993 experiment performed with the pretzel optics shows an unexpectedly large deviation which contradicts any expectation and was not confirmed in 1994 with the improved polarimeter when running in the same conditions. As all subsequent measurements showed differences compatible with zero it was decided to use, for 1993 and 1994, the largest possible estimated deviation as the correction with an error equal to the correction ( $0.3 \mathrm{MeV}$ on centre-of-mass energy). Without pretzel the expected possible deviation is negligible and for 1995 the error has been correspondingly reduced to $0.25 \mathrm{MeV}$ on the centre-of-mass energy and the correction set to zero.

\begin{tabular}{|c|c|c|c|}
\hline Year & Date & $E_{\mathrm{e}^{+}}-E_{\mathrm{e}^{-}}(\mathrm{MeV})$ & LEP operation \\
\hline 1993 & Nov. 15th & {$[0.5,3.2]$} & Pretzel \\
1994 & Jul. 15th & $0.4 \pm 0.4$ & Pretzel \\
& Aug. 1st & $0.0 \pm 0.2$ & Pretzel \\
1995 & Sep. 26th & $0.1 \pm 0.25$ & Bunch-Train \\
\hline
\end{tabular}

Table 17: Difference in energy between positron and electron beam as measured during the various years. In 1994 a dedicated positron polarimeter was installed, while in 1993 the measurement was performed with a modification of the electron polarimeter.

\section{Summary of the systematic errors on the 1993, 1994 and 1995 LEP centre-of-mass energies}

The luminosity-weighted energies at each IP are affected by a number of possible systematic error types.

There is an uncertainty in the average beam-energy determination at a given time arising from the accuracy with which the energy is tracked during a fill and from the precision in the definition of the absolute beam-energy for a given fill. The known contributions to the errors due to the tracking of the energy during a fill have been described in the preceding sections and the unknown ones estimated using the comparison of the model prediction with the measured changes during fills which had multiple calibrations.

In addition there are uncertainties from the RD measurement technique (see [3]) and errors on the corrections to convert the average beam-energy to the energy at the interaction points (see Section 10).

The full list of error components is summarized in Table 19. Note that for 1993 these supersede those given in [4]. In the same table an indication is also given of the contribution of each component to the error on the $m_{\mathrm{Z}}$ and $\Gamma_{\mathrm{Z}}$ measurements assuming a similar dataset for each experiment and an equal weight for the 1993 and 1995 energy scans; as this is only an approximation the final errors might differ slightly from those indicated.

The dominant error on $m_{Z}$ arises from the uncertainty in the dipole-field rise. The $\mathrm{Z}$ width is less sensitive to these uncertainties: the largest error contribution to $\Gamma_{Z}$ is the statistical contribution from uncalibrated fills. 
The systematic uncertainties have correlations between energy points, between IPs and between years. A $7 \times 7$ matrix was constructed which held the correlations between the $\mathrm{P}-2, \mathrm{P}$ and $\mathrm{P}+2$ energy points in 1993, 1994 and 1995 (see Table 18). This matrix takes into account the correlations between IPs in a manner which is correct if it is used to combine the data from the four LEP experiments. The full $28 \times 28$ matrix which contains the information about the errors and correlations between IP, years and energy points is given in the appendix.

\begin{tabular}{|c|c|c|c|c|c|c|c|}
\hline & $93 P-2$ & $93 P$ & $93 \mathrm{P}+2$ & $94 P$ & $95 \mathrm{P}-2$ & $95 P$ & $95 \mathrm{P}+2$ \\
\hline 93 P-2 & 11.71 & 7.60 & 6.73 & 5.06 & 1.66 & 1.41 & 1.45 \\
\hline $93 \mathbf{P}$ & & 44.81 & 6.96 & 5.67 & 1.30 & 1.43 & 1.33 \\
\hline $93 \mathrm{P}+2$ & & & 8.69 & 4.67 & 1.51 & 1.57 & 1.76 \\
\hline $94 \mathrm{P}$ & & & & 13.14 & 1.51 & 1.68 & 1.53 \\
\hline $95 \mathrm{P}-2$ & & & & & 3.17 & 1.53 & 1.49 \\
\hline $95 \mathrm{P}$ & & & & & & 29.08 & 1.79 \\
\hline $95 \mathrm{P}+2$ & & & & & & & 2.83 \\
\hline
\end{tabular}

Table 18: LEP energy common error matrix for the scan points of 1993, 1994 and 1995. The values are for centre-of-mass energy in units of $\mathrm{MeV}^{2}$.

\subsection{Periods with less precise energy calibration}

In 1993 and 1995, prior to the energy scans and in preparation for them, a substantial amount of data was collected at peak energies in conditions which were far from ideal from the point of view of controlling the systematics related to the LEP energy. In 1994, at the end of the year, a special accelerator configuration was tested in order to prepare for the bunch-train running scheme of 1995: LEP had for part of the time a special separation bump in IP4 and IP8. At the same time the existing superconducting RF cavities were also switched on to be tested. During these periods the recording of the RF status was erratic. The same model used to derive the energy for the other fills was used as much as possible for these fills.

For the 1993 pre-scan period (fills 1527 to 1624) the analysis described in [4] is still valid and hence a centre-of-mass energy error of $\pm 18 \mathrm{MeV}$ is retained. For the 1995 pre-scan period (fills 2614 to 2832) the accelerator parameters were better controlled than in 1993 and the main uncertainty stems from the evidence that one superconducting RF module voltage was not correctly logged. A centre-of-mass energy error of $\pm 10 \mathrm{MeV}$ is assigned for this period.

For the bunch-train test period, at the end of 1994, estimates of potential luminosity loss due to a vertical offset between the colliding beams, together with the theoretical upper limits on the vertical dispersion at the IPs, and the effects of missing RF records, lead to an error on the centre-of-mass energy of $\pm 20 \mathrm{MeV}$ (fills 2493 to 2551 ).

These error assignments are conservative: with such large values the correlations between years can safely be neglected. It is worth mentioning that within these special periods some physics fills were at energies significantly different (up to $500 \mathrm{MeV}$ in centre-of-mass) from the standard ones.

\subsection{Energies for the LEP runs before 1993}

The model of LEP used for determining the energies from 1993 onwards cannot be applied to the earlier datasets as some of the basic information was not logged and the quality of the accelerator diagnostic tools was lower. Systematic cross checks have been made to ensure that the times at which RD calibrations were performed in 1991 and 1992 were uniformly spread and therefore not biased in a significant way by the tidal and geological deformation cycles and by the field rise effects. The conclusion is that the published analysis had correctly evaluated the errors and is still adequate [6]. Given the large errors the year-to-year correlation can be neglected. 


\begin{tabular}{|c|c|c|c|c|c|c|c|c|c|c|c|c|c|c|c|c|}
\hline & $\mathrm{F}^{\mathrm{N}}$ & $\stackrel{8}{z}$ & $\stackrel{\infty}{\circ}$ & $\stackrel{20}{0}$ & $\overrightarrow{0}$ & $\breve{0}$ & $\breve{0}$ & $\overrightarrow{0}$ & $\stackrel{\overbrace{}}{0}$ & $\stackrel{20}{0}$ & $\dddot{\leftrightarrow}$ & $\overrightarrow{0}$ & $\ddot{\circ}$ & $\stackrel{\vartheta}{0}$ & $\overrightarrow{0}$ & $\stackrel{\sim}{0}$ \\
\hline & है & $\stackrel{\vec{D}}{\sum^{2}}$ & $\stackrel{20}{\circ}$ & $\stackrel{+}{0}$ & $\overrightarrow{0}$ & $\stackrel{\text { ֻै }}{\circ}$ & $\stackrel{\circ}{\circ}$ & $\stackrel{\circ}{\circ}$ & $\stackrel{\leftrightarrow}{\circ}$ & $\stackrel{29}{-1}$ & $\stackrel{\infty}{0}$ & $\overrightarrow{0}$ & $\stackrel{\sim}{0}$ & $\ddot{0}$ & $\stackrel{\sim}{?}$ & \\
\hline & $\stackrel{\bar{\varpi}}{\check{\varpi}}$ & 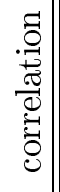 & $\dot{0}$ & $\begin{array}{l}\overrightarrow{0} \\
\dot{0}\end{array}$ & $\begin{array}{l}20 \\
\stackrel{1}{0} \\
0 \\
\dot{0}\end{array}$ & 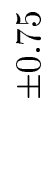 & $-\dot{-}$ & 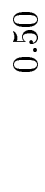 & $\stackrel{10}{\stackrel{2}{0}}$ & $\begin{array}{c}0 \\
\stackrel{2}{0} \\
0 \\
0\end{array}$ & $\stackrel{12}{\stackrel{10}{\circ}}$ & $\dot{0}$ & $\begin{array}{l}0 \\
0 \\
0 \\
0 \\
\dot{0} \\
\dot{0}\end{array}$ & $\begin{array}{l}r \\
? \\
0 \\
\infty \\
\infty \\
\ddot{0}\end{array}$ & $\begin{array}{l}0 \\
0 \\
0 \\
\dot{1} \\
\dot{0}\end{array}$ & \\
\hline & 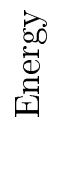 & 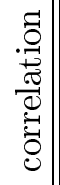 & $\dot{0}$ & $\begin{array}{l}\overleftrightarrow{0} \\
\dot{0}\end{array}$ & $\begin{array}{l}\stackrel{20}{12} \\
0\end{array}$ & 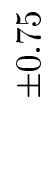 & $\vec{H}$ & $\vec{H}$ & $\stackrel{20}{\circ}$ & 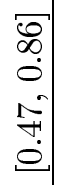 & $\begin{array}{l}120 \\
\stackrel{1}{0} \\
0\end{array}$ & $\begin{array}{l}10 \\
5 \\
0\end{array}$ & $\stackrel{20}{0}$ & 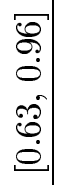 & $\begin{array}{l}2 \\
10 \\
0 \\
0 \\
0 \\
10 \\
0 \\
0\end{array}$ & \\
\hline \multirow{7}{*}{ 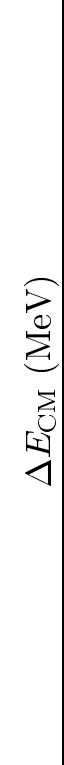 } & $\stackrel{\sim}{+}$ & 28 & $\stackrel{+}{0}$ & $\stackrel{20}{0}$ & $\stackrel{0}{0}$ & $\begin{array}{l}\text { ^. } \\
\text { i }\end{array}$ & $\begin{array}{l}0 \\
\dot{0} \\
1\end{array}$ & $\stackrel{\circ}{\circ}$ & $\stackrel{+1}{0}$ & $\begin{array}{l}0 \\
\dot{0}\end{array}$ & 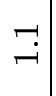 & $\dddot{0}$ & $\stackrel{\mathfrak{o}}{0}$ & $\tilde{0}$ & $\stackrel{\leftrightarrow}{0}$ & \\
\hline & $A$ & 20 & $\stackrel{0}{2}$ & $\stackrel{20}{0}$ & $\stackrel{\circ}{\circ}$ & 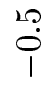 & $\begin{array}{l}0 \\
\dot{0} \\
1\end{array}$ & $\begin{array}{l}0 \\
\dot{0}\end{array}$ & $\stackrel{\leftrightarrow}{0}$ & $\stackrel{\bullet}{\circ}$ & $\stackrel{\circ}{-}$ & $\stackrel{+}{-}$ & $\stackrel{\sim}{\sigma}$ & $\check{0}$ & $\stackrel{\leftrightarrow}{0}$ & \\
\hline & $\stackrel{i}{i}$ & 22 & $\begin{array}{l}\infty \\
0 \\
0\end{array}$ & $\stackrel{10}{0}$ & $\stackrel{0}{0}$ & 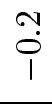 & $\begin{array}{l}0 \\
\dot{1} \\
1\end{array}$ & $\stackrel{?}{\dddot{1}}$ & $\stackrel{+}{0}$ & $\stackrel{0}{0}$ & : & $\stackrel{0}{\circ}$ & $\stackrel{\sim}{0}$ & $\stackrel{1}{0}$ & $\stackrel{r}{0}$ & \\
\hline & A & $\vec{\sigma}$ & $ت$ & $\stackrel{10}{0}$ & $\stackrel{\circ}{\circ}$ & ฯี & $\overrightarrow{0}$ & $\stackrel{1}{\circ}$ & テี & $\stackrel{\leftrightarrow}{\leftrightarrow}$ & 范 & $\dot{0}$ & $\stackrel{?}{0}$ & $\stackrel{0}{0}$ & $\ddot{\circ}$ & \\
\hline & $\begin{array}{r}\stackrel{\sim}{+} \\
\stackrel{+}{+}\end{array}$ & $\mathscr{g}$ & $\stackrel{g}{0}$ & $\stackrel{20}{0}$ & $\stackrel{\sim}{\sigma}$ & $\begin{array}{l}+ \\
\dot{1}\end{array}$ & $\stackrel{\Im}{o}$ & $\breve{i}_{1}^{+}$ & $\stackrel{+!}{0}$ & $\begin{array}{l}20 \\
\mathrm{~N}\end{array}$ & $\stackrel{0}{0}$ & $\dot{0}$ & $\stackrel{\leftrightarrow}{\circ}$ & $\stackrel{20}{0}$ & $\stackrel{+}{0}$ & \\
\hline & A & $\mathscr{\sigma}$ & $\begin{array}{l}9 \\
20\end{array}$ & $\stackrel{10}{0}$ & $\tilde{o}$ & $\stackrel{+}{0}$ & $\begin{array}{l}0 \\
0 \\
1\end{array}$ & $\stackrel{\circ}{0}$ & ت̈ & $\stackrel{0}{\circ}$ & $\dddot{\leftrightarrow}$ & $\dot{0}$ & $\stackrel{\Im}{0}$ & $\stackrel{20}{0}$ & $\stackrel{+!}{0}$ & \\
\hline & $\begin{array}{l}i \\
i \\
\sim\end{array}$ & $\mathscr{\varnothing}$ & $\stackrel{\square}{-}$ & $\stackrel{20}{0}$ & $\stackrel{\sim}{\sigma}$ & $\stackrel{\circ}{0}$ & $\stackrel{0}{\circ}$ & $\stackrel{\circ}{\circ}$ & $\overrightarrow{0}$ & $\begin{array}{l}\infty \\
\stackrel{\sim}{\sim}\end{array}$ & $\stackrel{0}{0}$ & 0 & $\stackrel{\leftrightarrow}{0}$ & $\stackrel{20}{0}$ & $\stackrel{+}{0}$ & \\
\hline \multicolumn{2}{|r|}{ U } & & 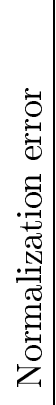 & 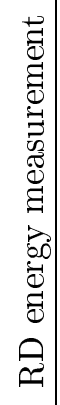 & 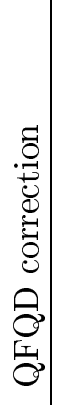 & 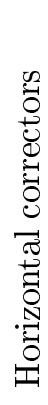 & 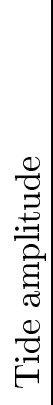 & 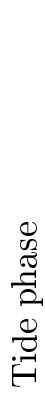 & 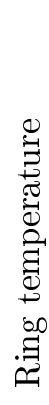 & 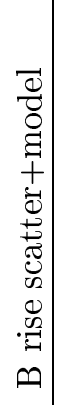 & 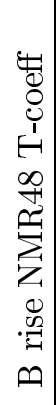 & 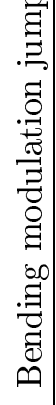 & 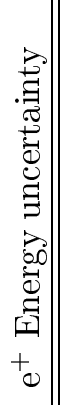 & 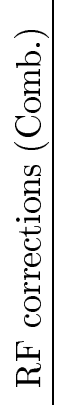 & 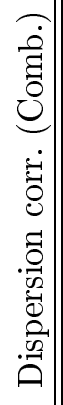 & 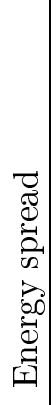 \\
\hline
\end{tabular}

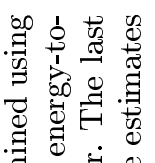

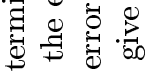

के

䒕芯范

कृ

वี

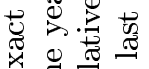

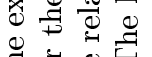

嵌焉

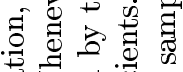

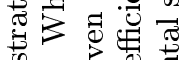

品

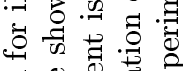

表获

o

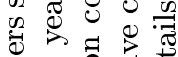

政.

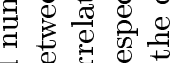

$\checkmark$ 过

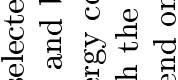

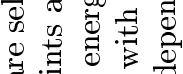

\%

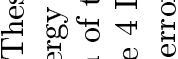

댕.

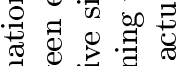

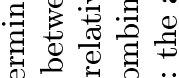

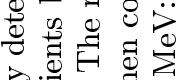

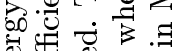

过

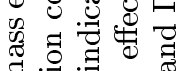

号要 $\frac{\pi}{0}$.

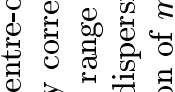

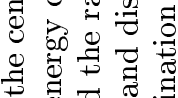

च

至

定

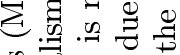

要

氙

पे

ส

명

की

$\ddot{g}$ 常

$\because$ ob

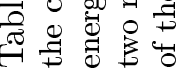


The LEP centre-of-mass energy spread $\sigma_{E_{\mathrm{CM}}}$ induces a shift $\delta \sigma$ in the measured cross section $(\sigma)$ proportional to its second derivative with respect to energy:

$$
\delta \sigma=-0.5 \frac{d^{2} \sigma}{d E^{2}} \sigma_{E_{\mathrm{CM}}}^{2}
$$

This in turn affects the measured width of the $\mathrm{Z}$ resonance. A spread of $55 \mathrm{MeV}$ requires a correction to the measured $\mathrm{Z}$ width of about $-4 \mathrm{MeV}$. This correction is essentially the same for all four LEP experiments and the corresponding error is fully correlated. Because the energy scans were approximately symmetric around the resonance peak, the effect on $m_{\mathrm{Z}}$ is negligible.

For the $1993 \mathrm{scan}, \sigma_{E_{\mathrm{CM}}}$ was evaluated with a $10 \%$ uncertainty, from measurements of the length of the luminous region in the LEP experiments. The resulting error of $1.0 \mathrm{MeV}$ on $\Gamma_{\mathrm{Z}}$ was the single largest systematic error on this quantity. The main systematic error originated from the uncertainty in the incoherent synchrotron tune $Q_{s}^{\text {inc. }}$.

The precision has been improved by a factor four for the 1995 scan, and retroactively for data

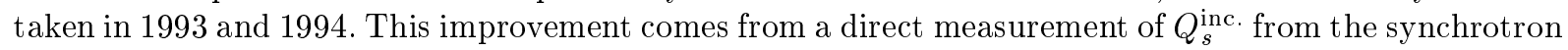
side-bands of the depolarizing resonance and an improved theoretical calculation of the expected beamenergy spread. In addition, the relationship between beam-energy spread and centre-of-mass energy spread in the presence of opposite-sign vertical dispersion has been investigated and corrected for. Full details of the analysis can be found in [28].

\subsection{Beam-energy spread calculations}

The rms beam-energy spread $\sigma_{E_{\mathrm{b}}}$ can be calculated for electrons in equilibrium between the radiation losses in the ring and the energy gain in the cavities [29]:

$$
\left(\frac{\sigma_{E_{\mathrm{b}}}}{E_{\mathrm{b}}}\right)^{2}=\frac{55}{32 \sqrt{3}} \frac{\hbar}{m_{\mathrm{e}} c} \gamma^{2} \frac{I_{3}}{J_{\mathrm{e}} I_{2}}
$$

where $E_{\mathrm{b}}$ is the beam energy, $\hbar$ the reduced Planck constant, $m_{\mathrm{e}}$ the electron mass, $c$ the speed of light, $\gamma$ the relativistic factor, $J_{\mathrm{e}}$ the energy damping partition number, $I_{2}=\oint G^{2} d s$ and $I_{3}=\oint|G|^{3} d s$ are the integrals around LEP ( $s$ being the local variable along the beam orbit) of $G(s)=e B(s) / p_{\mathrm{b}} c$, where $B(s)$ is the magnetic field, $p_{\mathrm{b}}$ the beam momentum and $e$ the electron charge.

The largest source of variation in $\sigma_{E_{\mathrm{b}}}$ comes from the excitation of the emittance wigglers. They are continuously trimmed to control the horizontal emittance and optimize the luminosity. Additional variations of $J_{\mathrm{e}}$, due to geometrical effects, were controlled with the beam-orbit-measurement system with a relative precision of $1 \%$. Variation of $\sigma_{E_{\mathrm{b}}}$ with wiggler current and $J_{\mathrm{e}}$ have been parametrised [30], allowing evaluation of $\sigma_{E_{\mathrm{b}}}$ as a function of time with an estimated precision of $2 \%$.

\subsection{Beam-energy spread from bunch-length measurements}

Another method to evaluate $\sigma_{E_{\mathrm{b}}}$ is to relate it to the rms longitudinal size $\left(\sigma_{z}\right)$ of the interaction region in the experiments. This quantity has the advantage of being continuously measured from the distribution of event vertices and is automatically luminosity-weighted. The relation between $\sigma_{E_{\mathrm{b}}}$ and $\sigma_{z}$ is [29],

$$
\sigma_{E_{\mathrm{b}}}=\frac{\sqrt{2} E_{\mathrm{b}}}{\alpha R_{\mathrm{L} E P}} Q_{s}^{\text {inc. }} \sigma_{z}
$$

where $\alpha$ is the LEP momentum compaction factor (see section 7 ), $R_{\mathrm{LEP}}$ is the average LEP accelerator

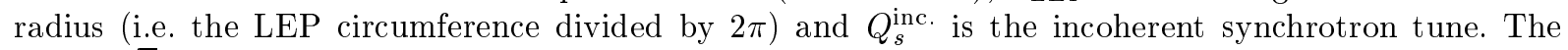
factor $\sqrt{2}$ relates the longitudinal size of the interaction region to that of the individual bunches.

The main uncertainty in this determination of $\sigma_{E_{\mathrm{b}}}$ comes from $Q_{s}^{\text {inc. }}$. The incoherent synchrotron tune is the number of energy oscillations per turn for individual beam particles. What is operationally 
measured are the longitudinal oscillations of full particle bunches, giving the coherent synchrotron tune, $Q_{s}^{\text {coh. }}$. Equation (22) is written in terms of $Q_{s}^{\text {inc. }}$. The relation between $Q_{s}^{\text {coh. }}$ and $Q_{s}^{\text {inc. is parametrized as }}$

$$
\frac{Q_{s}^{\text {coh. }}}{Q_{s}^{\text {inc. }}}=1-\kappa \frac{I}{I_{0}} \quad, \quad\left(I_{0}=300 \mu A\right)
$$

where $I$ is the bunch current and $\kappa$ is a complicated function of the longitudinal impedances of the machine elements which is difficult to evaluate analytically.

The incoherent synchrotron tune was measured in a dedicated experiment carried out at $\mathrm{P}+2$ in 1995 during an RD calibration. Depolarization was observed at the main resonance and at spin tunes different from the main one by $\pm Q_{s}$, identified as synchrotron sidebands. From the precisely determined

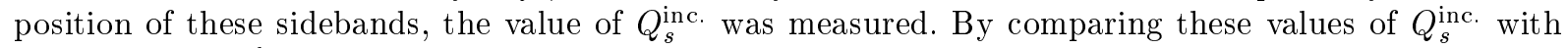

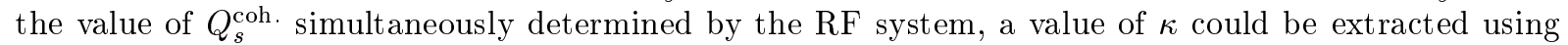
Eq. 23. The sidebands were determined for bunches with currents of 50 and $180 \mu \mathrm{A}$, showing agreement with Eq. 23 for a value of $\kappa=0.045 \pm 0.022$. The error is assigned from the frequency sweep interval (see Section 2.2) used in the RD measurements and the time variations of $Q_{s}^{\text {coh. }}$ during the experiment.

For each year of data-taking and each energy point, the beam-energy spread (luminosityweighted) was computed according to Eq. (22) using the rms of the $z$ distribution in ALEPH, the measured bunch currents and $Q_{s}^{\text {coh. }}$, and $\kappa=0.045$. For 1995 data, no difference between the different bunch families was observed.

Consistency checks of the two determinations of energy spread could be performed as a function of time for groups of events recorded in the ALEPH detector, showing consistency at the level of $\pm 2 \%$. The comparison is shown in Fig. 22 as a function of the most sensitive parameter, the wiggler current, showing again consistency at that level of precision.

\subsection{From beam-energy to centre-of-mass energy spread} formula:

The centre-of-mass energy spread $\sigma_{E_{\mathrm{CM}}}$ is related to the beam-energy spread $\sigma_{E_{\mathrm{b}}}$ by the simple

$$
\sigma_{E_{\mathrm{CM}}}=\sqrt{2} \sigma_{E_{\mathrm{b}}} C,(0 \leq C \leq 1)
$$

For colliding beams having no or identical energy dispersion (as in 1993 and 1994) the monochromatization factor $C$ is equal to 1 [22]. This is not the case for data taken in 1995 with the bunch train operation mode, where beams had opposite sign vertical energy dispersion leading to a more complicated relation between $\sigma_{E_{\mathrm{b}}}$ and $\sigma_{E_{\mathrm{CM}}}[25]$. This relation can be simplified to:

$$
\sigma_{E_{\mathrm{CM}}}^{2}=2 \sigma_{E_{\mathrm{b}}}^{2}\left[1+\left(\frac{D_{y} \frac{\sigma_{E_{\mathrm{b}}}}{E_{\mathrm{b}}}}{2 \sigma_{y}}\right)^{2}\right]^{-1}
$$

where $D_{y}$ is the opposite sign vertical energy dispersion and $\sigma_{y}$ is the vertical beam size.

The possible beam dispersion differences at the IP for the 1993 and 1994 running periods have been evaluated [31]. The reduction on the centre-of-mass energy spread is estimated to be less than $0.2 \%$. This corresponds to a change of $0.1 \mathrm{MeV}$ on $\sigma_{E_{\mathrm{CM}}}$, and is therefore neglected. For the 1995 running period this correction amounts to about $2 \%$ and is different for each IP. This was calculated using the measurements of dispersions [25] described in Section 10.3.

The LEP centre-of-mass energy spread is given in table 20 for the different years where also the luminosity-weighted average squares, which are needed in Eq. (20), are shown. The measured energy spread increases with energy, as expected.

LEP centre-of-mass energy variations increase the centre-of-mass energy spread. The rms of the LEP centre-of-mass energy distribution for each year, experiment and energy point has to be added in quadrature to the numbers given in Table 20 .

\subsection{Systematic errors on the LEP centre-of-mass energy spread}

A full estimate of systematic errors was performed for the determination using bunch length as follows. The resulting evaluation of $\pm 2 \%$ is similar to the quality of the consistency with the theoretical calculation. The following parameters contribute to the systematic error on the LEP centre-of-mass energy spread. 


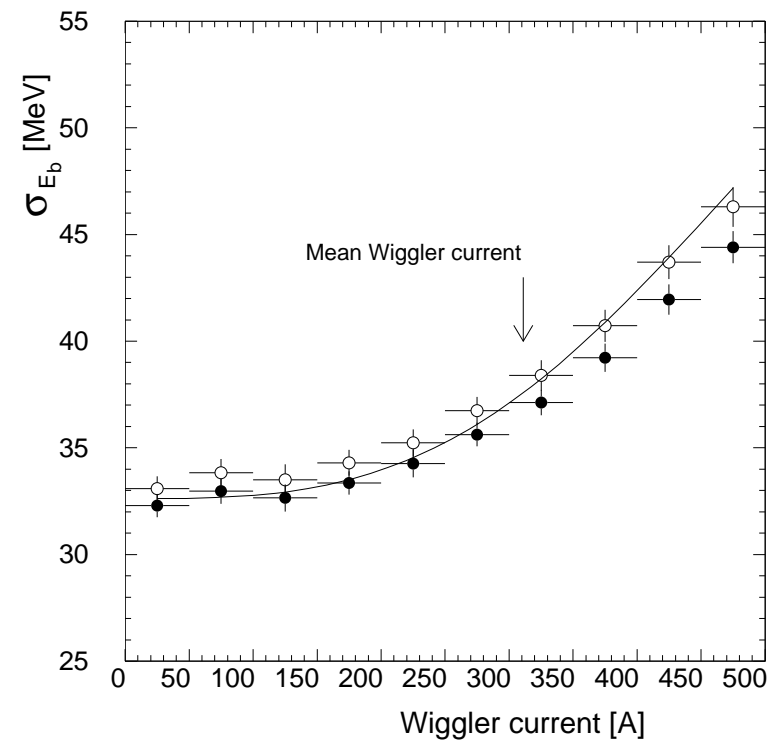

Figure 22: 1994 data. Comparison of the two evaluations of $\sigma_{E_{\mathrm{b}}}$ as a function of wiggler current. The 'theoretical'

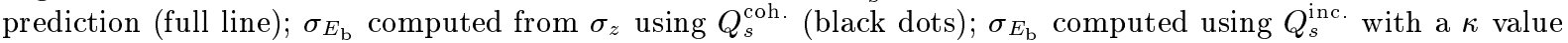
of 0.045 (open dots).

\begin{tabular}{||c||c|c|cccc|}
\hline \multicolumn{1}{||||}{} & \multicolumn{6}{c|}{$\sigma_{\mathrm{E}_{\mathrm{CM}}}\left(\sigma_{\mathrm{E}_{\mathrm{CM}}}^{2}\right)$ in $\mathrm{MeV}\left(\mathrm{MeV}^{2}\right)$} \\
\hline & 1993 & 1994 & & \multicolumn{2}{c}{1995} & IP8 \\
\hline \hline $\mathrm{P}-2$ & $54.6(3022)$ & & $55.0(3084)$ & $55.7(3154)$ & $55.7(3154)$ & $55.7(3159)$ \\
$\mathrm{P}$ & $55.4(3108)$ & $54.9(3044)$ & $55.6(3128)$ & $56.2(3198)$ & $56.2(3199)$ & $56.3(3204)$ \\
$\mathrm{P}+2$ & $55.6(3120)$ & & $56.0(3196)$ & $56.6(3269)$ & $56.7(3269)$ & $56.7(3274)$ \\
\hline
\end{tabular}

Table 20: Centre-of-mass energy spread for the different years and interaction points. The numbers in brackets are the average $\sigma_{\mathrm{E}_{\mathrm{CM}}}^{2}$ including the effect of varying energy spread during a fill due to changes in the wiggler magnets currents. These are the numbers needed since the cross-section correction depends at leading order on $\sigma_{\mathrm{E}_{\mathrm{CM}}}^{2}$. 


\begin{tabular}{|l||c|c|c|}
\hline \multicolumn{1}{|c||}{} & \multicolumn{3}{c|}{$\sigma_{\mathrm{E}_{\mathrm{CM}}}$ in $\mathrm{MeV}$} \\
\hline & 1990 & 1991 & 1992 \\
\hline \hline $\mathrm{P}-3$ & 47. & 43. & \\
$\mathrm{P}-2$ & 48. & 44. & \\
$\mathrm{P}-1$ & 49. & 45. & \\
$\mathrm{P}$ & 50. & 46. & 51. \\
$\mathrm{P}+1$ & 51. & 47. & \\
$\mathrm{P}+2$ & 52. & 48. & \\
$\mathrm{P}+3$ & 53. & 49. & \\
\hline
\end{tabular}

Table 21: Centre-of-mass energy spread for the years 1990, 1991, 1992. To be noted that the Z scans before 1993 consisted of 6 off-peak points: here \pm 1 and \pm 3 means respectively $\pm 0.9 \mathrm{GeV}$ and $\pm 3 \cdot 0.9 \mathrm{GeV}$ on either side of the $\mathrm{Z}$ peak for 1991 and 1992, while for 1990 the step is an integer number of GeV.

- The LEP momentum compaction factor $\alpha$, was measured to be $(1.86 \pm 0.02) \cdot 10^{-4}$. The $1 \%$ error on this parameter directly translates into a $1 \%$ error on $\sigma_{E_{\mathrm{b}}}: 0.4 \mathrm{MeV}$.

- The difference between the measured and the computed $Q_{s}^{\text {coh. }}$ is -0.00034 for the 1995 scan [20] and 0.0001 for the 1993 scan [32]. It is a $10^{-3}$ effect at maximum (mean value of $\left.Q_{s}=0.065\right)$ and therefore can be neglected.

- The beam-energy variations within a fill have not been included in the above calculations. However it is less than a $10^{-3}$ effect and can be neglected.

- The error on $\kappa$ is the dominant error, resulting in an error of $0.7 \mathrm{MeV}$ on $\sigma_{E_{\mathrm{b}}}$ and of 1.1 $\mathrm{MeV}$ on $\sigma_{E_{\mathrm{CM}}}$.

- The errors on the opposite-sign vertical dispersion, described in section 10.3, yield an error of the order of 0.01 on the monochromatization factor $C$, which leads to a $0.6 \mathrm{MeV}$ error on $\sigma_{E_{\mathrm{CM}}}$, for 1995 data. This uncertainty is negligible for earlier years.

From the above discussion, the error on the LEP centre-of-mass energy spread is $1.1 \mathrm{MeV}$ for 1993 and 1994. For 1995 this error is increased to $1.3 \mathrm{MeV}$ due to vertical beam-energy dispersion. These numbers are correlated between years and experiments. The contribution to the corresponding error on $\Gamma_{\mathrm{Z}}$ is $\Delta \Gamma_{\mathrm{Z}} \approx 0.2 \mathrm{MeV}$.

\subsection{Energy spread for LEP runs before 1993}

To insure a consistent set of values for the analysis of LEP data taken before 1993, the values of the energy spread to be used for the years previous to 1993 are presented here. These values supersede those given in [33]. The Table 21 summarizes the results for the years 1990, 1991 and 1992. The values from 1989 are the same as 1990. Since no data on the time-dependence of the energy spread exists, the quantity $\sigma_{E_{\mathrm{CM}}}^{2}$ is assumed to be equal to $\left(\sigma_{E_{\mathrm{CM}}}\right)^{2}$, where $\sigma_{E_{\mathrm{CM}}}$ is taken from Table 21 .

The difference in values between 1990 and 1991 is a result of the beams being more centered in the LEP quadrupoles during 1991. In 1992, wiggler magnets were used in LEP to increase the beam size in order to maintain a higher luminosity, which also increased the energy spread.

Owing to the less accurate logging of vital information, the error the center-of-mass energy spread is taken to be $3 \mathrm{MeV}$ for all years prior to 1993 .

\section{Conclusions}

The 1995 energy scan and the improved accelerator instrumentation have allowed a significant advance in the understanding of the energy of the LEP beams. The improved understanding of the 
thermal behaviour of the LEP dipoles and newly discovered effects of parasitic currents flowing on the LEP beam-pipe have improved the modelling of the LEP energies. All the datasets since 1993 have been analysed and the final LEP energies determined with small, but significant, changes with respect to the published 1993 results.

The precision of the centre-of-mass energy spread determination has been improved by more than a factor of four reducing significantly what used to be one of the dominant sources of error in the $\mathrm{Z}$ width.

The overall contribution of the energy uncertainties to the combined LEP $m_{\mathrm{Z}}$ and $\Gamma_{\mathrm{Z}}$ measurement can be estimated to be $\Delta m_{\mathrm{Z}} \approx 1.9 \mathrm{MeV}$ and $\Delta \Gamma_{\mathrm{Z}} \approx 1.2 \mathrm{MeV}$ on the assumption that datasets from all experiments and both scans enter with equal weight. A full fit to the experimental cross-sections using the error matrix of Appendix A is expected to yield different errors.

\section{Acknowledgements}

We thank the SL Division of CERN for the excellent performance of the LEP collider. The careful work and assistance of many technical groups in the AS, MT and ST Divisions has been essential to reach the performance described in this paper. We acknowledge many useful discussions with J. Jowett, E. Keil, W. Herr and S. Myers. We also acknowledge the support of the Particle Physics and Astronomy Research Council, UK. 


\section{Appendix}

\section{A The global error matrix}

The LEP energy error matrix representing the errors and corresponding correlations between energy points, years and IPs for 1993, 1994 and 1995 is given. The values are for centre-of-mass energy in units of $\mathrm{MeV}^{2}$. The prefixes L, A, O, D stand, respectively, for IP2, IP4, IP6 and IP8, while the other part of the column and row labels represents the year $(1993,1994,1995)$ and the energy point.

$\begin{array}{lrrrrrrr} & \text { L93-2 } & \text { L93P } & \text { L93+2 } & \text { L94P } & \text { L95-2 } & \text { L95P } & \text { L95+2 } \\ \text { L93-2 } & 12.59 & 8.32 & 7.45 & 5.59 & 2.05 & 1.80 & 1.84 \\ \text { L93P } & 8.32 & 45.69 & 7.68 & 6.20 & 1.69 & 1.82 & 1.72 \\ \text { L93+2 } & 7.45 & 7.68 & 9.57 & 5.20 & 1.90 & 1.96 & 2.15 \\ \text { L94P } & 5.59 & 6.20 & 5.20 & 14.30 & 1.90 & 2.07 & 1.92 \\ \text { L95-2 } & 2.05 & 1.69 & 1.90 & 1.90 & 4.49 & 2.34 & 2.30 \\ \text { L95P } & 1.80 & 1.82 & 1.96 & 2.07 & 2.34 & 30.40 & 2.60 \\ \text { L95+2 } & 1.84 & 1.72 & 2.15 & 1.92 & 2.30 & 2.60 & 4.15 \\ \text { A93-2 } & 11.53 & 7.43 & 6.56 & 4.91 & 1.61 & 1.36 & 1.40 \\ \text { A93P } & 7.43 & 44.63 & 6.79 & 5.52 & 1.25 & 1.38 & 1.28 \\ \text { A93+2 } & 6.56 & 6.79 & 8.51 & 4.52 & 1.46 & 1.52 & 1.71 \\ \text { A94P } & 4.91 & 5.52 & 4.52 & 12.87 & 1.47 & 1.64 & 1.49 \\ \text { A95-2 } & 1.61 & 1.25 & 1.46 & 1.47 & 3.25 & 1.57 & 1.53 \\ \text { A95P } & 1.36 & 1.38 & 1.52 & 1.64 & 1.57 & 29.16 & 1.83 \\ \text { A95+2 } & 1.40 & 1.28 & 1.71 & 1.49 & 1.53 & 1.83 & 2.91 \\ \text { O93-2 } & 11.76 & 7.78 & 6.91 & 5.29 & 1.81 & 1.56 & 1.60 \\ \text { O93P } & 7.78 & 44.86 & 7.14 & 5.90 & 1.45 & 1.58 & 1.48 \\ \text { O93+2 } & 6.91 & 7.14 & 8.74 & 4.90 & 1.66 & 1.72 & 1.91 \\ \text { O94P } & 5.29 & 5.90 & 4.90 & 13.15 & 1.66 & 1.83 & 1.68 \\ \text { O95-2 } & 1.63 & 1.27 & 1.48 & 1.48 & 2.90 & 1.42 & 1.38 \\ \text { O95P } & 1.38 & 1.40 & 1.54 & 1.65 & 1.42 & 28.81 & 1.68 \\ \text { O95+2 } & 1.42 & 1.30 & 1.73 & 1.50 & 1.38 & 1.68 & 2.56 \\ \text { D93-2 } & 11.53 & 7.43 & 6.56 & 4.91 & 1.61 & 1.36 & 1.40 \\ \text { D93P } & 7.43 & 44.63 & 6.79 & 5.52 & 1.25 & 1.38 & 1.28 \\ \text { D93+2 } & 6.56 & 6.79 & 8.51 & 4.52 & 1.46 & 1.52 & 1.71 \\ \text { D94P } & 4.91 & 5.52 & 4.52 & 12.87 & 1.47 & 1.64 & 1.49 \\ \text { D95-2 } & 1.61 & 1.25 & 1.46 & 1.47 & 3.26 & 1.58 & 1.54 \\ \text { D95P } & 1.36 & 1.38 & 1.52 & 1.64 & 1.58 & 29.17 & 1.84 \\ \text { D95+2 } & 1.40 & 1.28 & 1.71 & 1.49 & 1.54 & 1.84 & 2.92\end{array}$




$\begin{array}{lrrrrrrr} & \text { A93-2 } & \text { A93P } & \text { A93+2 } & \text { A94P } & \text { A95-2 } & \text { A95P } & \text { A95+2 } \\ \text { L93-2 } & 11.53 & 7.43 & 6.56 & 4.91 & 1.61 & 1.36 & 1.40 \\ \text { L93P } & 7.43 & 44.63 & 6.79 & 5.52 & 1.25 & 1.38 & 1.28 \\ \text { L93+2 } & 6.56 & 6.79 & 8.51 & 4.52 & 1.46 & 1.52 & 1.71 \\ \text { L94P } & 4.91 & 5.52 & 4.52 & 12.87 & 1.47 & 1.64 & 1.49 \\ \text { L95-2 } & 1.61 & 1.25 & 1.46 & 1.47 & 3.25 & 1.57 & 1.53 \\ \text { L95P } & 1.36 & 1.38 & 1.52 & 1.64 & 1.57 & 29.16 & 1.83 \\ \text { L95+2 } & 1.40 & 1.28 & 1.71 & 1.49 & 1.53 & 1.83 & 2.91 \\ \text { A93-2 } & 11.71 & 7.56 & 6.69 & 5.06 & 1.62 & 1.37 & 1.41 \\ \text { A93P } & 7.56 & 44.81 & 6.92 & 5.67 & 1.26 & 1.39 & 1.29 \\ \text { A93+2 } & 6.69 & 6.92 & 8.69 & 4.67 & 1.47 & 1.53 & 1.72 \\ \text { A94P } & 5.06 & 5.67 & 4.67 & 13.37 & 1.48 & 1.65 & 1.50 \\ \text { A95-2 } & 1.62 & 1.26 & 1.47 & 1.48 & 3.33 & 1.51 & 1.47 \\ \text { A95P } & 1.37 & 1.39 & 1.53 & 1.65 & 1.51 & 29.24 & 1.77 \\ \text { A95+2 } & 1.41 & 1.29 & 1.72 & 1.50 & 1.47 & 1.77 & 2.99 \\ \text { O93-2 } & 11.53 & 7.43 & 6.56 & 4.91 & 1.61 & 1.36 & 1.40 \\ \text { O93P } & 7.43 & 44.63 & 6.79 & 5.52 & 1.25 & 1.38 & 1.28 \\ \text { O93+2 } & 6.56 & 6.79 & 8.51 & 4.52 & 1.46 & 1.52 & 1.71 \\ \text { O94P } & 4.91 & 5.52 & 4.52 & 12.87 & 1.47 & 1.64 & 1.49 \\ \text { O95-2 } & 1.61 & 1.25 & 1.46 & 1.47 & 2.81 & 1.34 & 1.30 \\ \text { O95P } & 1.36 & 1.38 & 1.52 & 1.64 & 1.34 & 28.72 & 1.60 \\ \text { O95+2 } & 1.40 & 1.28 & 1.71 & 1.49 & 1.30 & 1.60 & 2.47 \\ \text { D93-2 } & 11.51 & 7.41 & 6.54 & 4.90 & 1.60 & 1.35 & 1.39 \\ \text { D93P } & 7.41 & 44.61 & 6.77 & 5.51 & 1.24 & 1.37 & 1.27 \\ \text { D93+2 } & 6.54 & 6.77 & 8.49 & 4.51 & 1.45 & 1.51 & 1.70 \\ \text { D94P } & 4.90 & 5.51 & 4.51 & 12.86 & 1.46 & 1.63 & 1.48 \\ \text { D95-2 } & 1.60 & 1.24 & 1.45 & 1.46 & 3.06 & 1.46 & 1.42 \\ \text { D95P } & 1.35 & 1.37 & 1.51 & 1.63 & 1.46 & 28.97 & 1.72 \\ \text { D95+2 } & 1.39 & 1.27 & 1.70 & 1.48 & 1.42 & 1.72 & 2.72\end{array}$




\section{O93-2 $\quad$ O93P $\quad$ O93+2 $\quad$ O94P $\quad$ O95-2 $\quad$ O95P $\quad$ O95+2}

$\begin{array}{lrrrrrrr}\text { L93-2 } & 11.76 & 7.78 & 6.91 & 5.29 & 1.63 & 1.38 & 1.42 \\ \text { L93P } & 7.78 & 44.86 & 7.14 & 5.90 & 1.27 & 1.40 & 1.30 \\ \text { L93+2 } & 6.91 & 7.14 & 8.74 & 4.90 & 1.48 & 1.54 & 1.73 \\ \text { L94P } & 5.29 & 5.90 & 4.90 & 13.15 & 1.48 & 1.65 & 1.50 \\ \text { L95-2 } & 1.81 & 1.45 & 1.66 & 1.66 & 2.90 & 1.42 & 1.38 \\ \text { L95P } & 1.56 & 1.58 & 1.72 & 1.83 & 1.42 & 28.81 & 1.68 \\ \text { L95+2 } & 1.60 & 1.48 & 1.91 & 1.68 & 1.38 & 1.68 & 2.56 \\ \text { A93-2 } & 11.53 & 7.43 & 6.56 & 4.91 & 1.61 & 1.36 & 1.40 \\ \text { A93P } & 7.43 & 44.63 & 6.79 & 5.52 & 1.25 & 1.38 & 1.28 \\ \text { A93+2 } & 6.56 & 6.79 & 8.51 & 4.52 & 1.46 & 1.52 & 1.71 \\ \text { A94P } & 4.91 & 5.52 & 4.52 & 12.87 & 1.47 & 1.64 & 1.49 \\ \text { A95-2 } & 1.61 & 1.25 & 1.46 & 1.47 & 2.81 & 1.34 & 1.30 \\ \text { A95P } & 1.36 & 1.38 & 1.52 & 1.64 & 1.34 & 28.72 & 1.60 \\ \text { A95+2 } & 1.40 & 1.28 & 1.71 & 1.49 & 1.30 & 1.60 & 2.47 \\ \text { O93-2 } & 12.59 & 8.32 & 7.45 & 5.59 & 1.71 & 1.46 & 1.50 \\ \text { O93P } & 8.32 & 45.69 & 7.68 & 6.20 & 1.35 & 1.48 & 1.38 \\ \text { O93+2 } & 7.45 & 7.68 & 9.57 & 5.20 & 1.56 & 1.62 & 1.81 \\ \text { O94P } & 5.59 & 6.20 & 5.20 & 14.30 & 1.56 & 1.73 & 1.58 \\ \text { O95-2 } & 1.71 & 1.35 & 1.56 & 1.56 & 3.36 & 1.64 & 1.60 \\ \text { O95P } & 1.46 & 1.48 & 1.62 & 1.73 & 1.64 & 29.27 & 1.90 \\ \text { O95+2 } & 1.50 & 1.38 & 1.81 & 1.58 & 1.60 & 1.90 & 3.02 \\ \text { D93-2 } & 11.53 & 7.43 & 6.56 & 4.91 & 1.61 & 1.36 & 1.40 \\ \text { D93P } & 7.43 & 44.63 & 6.79 & 5.52 & 1.25 & 1.38 & 1.28 \\ \text { D93+2 } & 6.56 & 6.79 & 8.51 & 4.52 & 1.46 & 1.52 & 1.71 \\ \text { D94P } & 4.91 & 5.52 & 4.52 & 12.87 & 1.47 & 1.64 & 1.49 \\ \text { D95-2 } & 1.61 & 1.25 & 1.46 & 1.47 & 2.82 & 1.35 & 1.31 \\ \text { D95P } & 1.36 & 1.38 & 1.52 & 1.64 & 1.35 & 28.73 & 1.61 \\ \text { D95+2 } & 1.40 & 1.28 & 1.71 & 1.49 & 1.31 & 1.61 & 2.48\end{array}$




$\begin{array}{lrrrrrrr} & \text { D93-2 } & \text { D93P } & \text { D93+2 } & \text { D94P } & \text { D95-2 } & \text { D95P } & \text { D95+2 } \\ \text { L93-2 } & 11.53 & 7.43 & 6.56 & 4.91 & 1.61 & 1.36 & 1.40 \\ \text { L93P } & 7.43 & 44.63 & 6.79 & 5.52 & 1.25 & 1.38 & 1.28 \\ \text { L93+2 } & 6.56 & 6.79 & 8.51 & 4.52 & 1.46 & 1.52 & 1.71 \\ \text { L94P } & 4.91 & 5.52 & 4.52 & 12.87 & 1.47 & 1.64 & 1.49 \\ \text { L95-2 } & 1.61 & 1.25 & 1.46 & 1.47 & 3.26 & 1.58 & 1.54 \\ \text { L95P } & 1.36 & 1.38 & 1.52 & 1.64 & 1.58 & 29.17 & 1.84 \\ \text { L95+2 } & 1.40 & 1.28 & 1.71 & 1.49 & 1.54 & 1.84 & 2.92 \\ \text { A93-2 } & 11.51 & 7.41 & 6.54 & 4.90 & 1.60 & 1.35 & 1.39 \\ \text { A93P } & 7.41 & 44.61 & 6.77 & 5.51 & 1.24 & 1.37 & 1.27 \\ \text { A93+2 } & 6.54 & 6.77 & 8.49 & 4.51 & 1.45 & 1.51 & 1.70 \\ \text { A94P } & 4.90 & 5.51 & 4.51 & 12.86 & 1.46 & 1.63 & 1.48 \\ \text { A95-2 } & 1.60 & 1.24 & 1.45 & 1.46 & 3.06 & 1.46 & 1.42 \\ \text { A95P } & 1.35 & 1.37 & 1.51 & 1.63 & 1.46 & 28.97 & 1.72 \\ \text { A95+2 } & 1.39 & 1.27 & 1.70 & 1.48 & 1.42 & 1.72 & 2.72 \\ \text { O93-2 } & 11.53 & 7.43 & 6.56 & 4.91 & 1.61 & 1.36 & 1.40 \\ \text { O93P } & 7.43 & 44.63 & 6.79 & 5.52 & 1.25 & 1.38 & 1.28 \\ \text { O93+2 } & 6.56 & 6.79 & 8.51 & 4.52 & 1.46 & 1.52 & 1.71 \\ \text { O94P } & 4.91 & 5.52 & 4.52 & 12.87 & 1.47 & 1.64 & 1.49 \\ \text { O95-2 } & 1.61 & 1.25 & 1.46 & 1.47 & 2.82 & 1.35 & 1.31 \\ \text { O95P } & 1.36 & 1.38 & 1.52 & 1.64 & 1.35 & 28.73 & 1.61 \\ \text { O95+2 } & 1.40 & 1.28 & 1.71 & 1.49 & 1.31 & 1.61 & 2.48 \\ \text { D93-2 } & 11.71 & 7.56 & 6.69 & 5.06 & 1.62 & 1.37 & 1.41 \\ \text { D93P } & 7.56 & 44.81 & 6.92 & 5.67 & 1.26 & 1.39 & 1.29 \\ \text { D93+2 } & 6.69 & 6.92 & 8.69 & 4.67 & 1.47 & 1.53 & 1.72 \\ \text { D94P } & 5.06 & 5.67 & 4.67 & 13.37 & 1.48 & 1.65 & 1.50 \\ \text { D95-2 } & 1.62 & 1.26 & 1.47 & 1.48 & 3.41 & 1.54 & 1.50 \\ \text { D95P } & 1.37 & 1.39 & 1.53 & 1.65 & 1.54 & 29.32 & 1.80 \\ \text { D95+2 } & 1.41 & 1.29 & 1.72 & 1.50 & 1.50 & 1.80 & 3.07\end{array}$




\section{References}

(1) Much of the information relevant to this paper is contained in notes of the SL division at CERN. Copies of these notes can be obtained from the CERN archives or via the archives interface at the CERN World Wide Web site http://www.cern.ch.

[1] L. Knudsen et al., 'First observation of transverse beam polarization in LEP', Phys. Lett. B270 (1991) 97

[2] L. Arnaudon et al, 'Measurement of LEP beam energy by resonant spin depolarization', Phys. Lett. B284 (1992) 431

[3] L. Arnaudon et al., 'Accurate determination of the LEP beam-energy by resonant depolarization', Z. Phys. C66 (1995) 45

[4] R. Assmann et al., LEP Energy Working Group, 'The energy calibration of LEP in the 1993 scan', Z. Phys. C66 (1995) 567

[5] The first published evidence of vagabond currents which we have been able to find is in Nature, Vol.58, NO. 1509, 29 September, 1898, p. 533. The few paragraphs included in a report of 'Physics at the British Association' describe well the source of the phenomena which have affected LEP.

[6] L. Arnaudon et al., 'Measurement of the mass of the $\mathrm{Z}$ boson and the energy calibration of LEP', Phys. Lett. B307 (1993) 187;

L. Arnaudon et al.,'The Energy Calibration of LEP in 1992', CERN SL/93-21 (DI), April 1993

[7] A.A. Sokolov and I.M. Ternov: 'On Polarization and spin effects in the theory of synchrotron radiation'. Sov. Phys. Dokl. 8 (1964) 1203

[8] M. Geitz, 'Investigation of the environmental effects on the LEP beam-energy', Diploma thesis, RWTH Aachen, PITHA Nr. 96/14, also published as CERN SL Note/96-50 (BI);

B. Dehning, A. Drees, M. Geitz, 'A Study of the Magnetic Dipole Field of LEP during the 1995 Energy Scan', 1996, CERN SL-96-054 ;

G. Brun et al.,'A newly observed Effect affects the LEP Beam Energy', CERN SL-96-036, also published in Proc. 1996 European Particle Accelerator Conference, editors S. Myers, A. Pacheco, R. Pascual, C.Petit-Jean-Genaz and J Poole, Institute of Physics, Bristol, 1996.

[9] J. Billan et al., 'Field display system for the forecast of beam momentum and betatron frequencies at LEP', Particle Accelerators 29 (1990) 215

[10] G. Vismara, 'The new front-end narrow-band electronics for the LEP Beam Orbit Measurement system', Proc. 1994 European Particle Accelerator Conference, London, World Scientific, London, July 1994

[11] P. Melchior: 'Tidal Interactions in the Earth Moon System', Communications, Serie B, No. 160, IUG General Assembly, Vienna, August 1991. Fortran code courtesy of P. Melchior, Observatoire Royal de Belgique

[12] L. Arnaudon et al., 'Effects of terrestrial tides on the LEP beam energy', NIM A357 (1995) 249-252.

[13] E. Bravin et al., 'The influence of train leakage currents on the LEP dipole field', CERN SL/97$47(\mathrm{BI})$

[14] K.A. Drees, 'High precision measurements of the LEP centre of mass energies during the 1993 and 1995 Z resonance Scans', Ph.D. thesis, Bergische Universität und Gesamtschule Wuppertal, WU-DIS 97-5 (1997)

[15] J. Billan, 'Influence of mortar-induced stresses on the magnetic characteristics of the LEP dipole cores', IEEE Transaction on Magnetics, Vol. 24, No. 2, March 1988

[16] E. Bravin, B. Dehning, A. Drees, G. Mugnai, 'Model of the dipole field variations in LEP bending magnets', CERN SL Note/97-46 (BI)

[17] The measurement of the momentum compaction factor is shown in Fig. 6, p. 48 in Ref.[3].

[18] J. Wenninger, 'Measurement of tidal deformation of the LEP ring with closed orbits', CERN SL Note/96-22 (OP)

[19] J. Wenninger, 'Orbit corrector magnets and beam-energy', CERN SL Note/97-06

[20] M.D. Hildreth, 'A study of RF effects on bunch-trains in the 1995 running', LEP Energy Working Group/97-02

[21] M.D. Hildreth, 'A systematic check of superconducting cavity alignment using LEP beams', LEP Energy Working Group/97-04

[22] J.M. Jowett, J. Wenninger, J. Yamartino, 'Influence of dispersion and collision offsets on the centreof-mass energy at LEP', CERN SL Note/95-46 (OP) 
[23] E. Keil, 'Truly self-consistent treatment of the side effects with bunch-trains', CERN SL/95-75

[24] LEP energy working group, 'LEP center-of-mass energies in presence of opposite sign vertical dispersion in bunch-train operation' CERN SL Note/95-77(BI)

[25] P. Puzo, J. Yamartino, 'Preliminary centre-of-mass energy corrections from dispersion and collision offsets for the 1995 scan', LEP Energy Working Group/96-01;

M. Böge et al.,'Measurements of Collision Offsets and Difference in vertical Dispersion at the LEP Interaction Points', CERN SL-96-035, also published in Proc. 1996 European Particle Accelerator Conference, Editors S. Myers, A. Pacheco, R. Pascual, C.Petit-Jean-Genaz and J Poole Institute of Physics, Bristol, 1996.

[26] H. Grote and F.C. Iselin, 'The MAD program', version 8.16, CERN SL/90-13 (AP) (rev.4, March $19,1995)$

[27] G. Burtin et al., 'Performance and operational experience of the LEP synchrotron light telescope', Proc. Particle Accelerator Conference 1993, Washington, 1993, IEEE, 445 Hose Lane, Piscataway, NJ 088544150, Vol.3, 2495

[28] E. Lancon and A. Blondel, 'Determination of the LEP Energy Spread Using Experimental Constraints', LEP Energy Working Group note 96-07.

[29] M. Sands, 'The Physics of electron storage rings, an introduction', SLAC-121, 1970

[30] J.M. Jowett, Private communication

[31] J. Wenninger, 'Centre-of-mass energy shifts from difference dispersion and collisions offsets in pretzel operation at LEP', CERN SL/95-64 (OP)

[32] G. Quast, 'Present understanding of RF effects', Memo to LEP Energy Working Group 93-2, 6 April, 1993

[33] L. Arnaudon et al., The LEP energy working group, 'The Energy Calibration of LEP in 1991', CERN-PPE/92-125, CERN-SL/92-37(DI), also published in Proc. $26^{\text {th }}$ ICHEP, Dallas, TX, 1992 